MODELING, IDENTIFICATION AND CONTROL, 2000, vOL. 21, NO. 3, 129-184

doi:10.4173/mic.2000.3.1

\title{
Nonlinear Passive Control and Observer Design for Ships
}

\author{
THOR I. FOSSEN $\dagger$ \\ Keywords: Passivity, nonlinear control, observers, ship control, marine systems
}

Starting with passivity of the ambient water-ship system this article proceeds with nonlinear observer design, design of dynamic ship positioning systems and weather optimal positioning control systems exploiting the passivity properties of the vessel and the surrounding water. The article gives an overview of methods for passive ship control and observer design.

\section{Introduction}

In this paper an overview of passive ship control systems design is presented. Passivity as a tool for ship control was first applied in the 1990s even though passivity concepts have been widely used and recognized as a physical and intuitive design methodology for mechanical systems like robots, pendulums, motors etc. Before this, see Berghuis (1993), Berghuis and Nijmeijer (1993), Canudas de Wit et al. (1992), Landau and Horowitz (1989), Loria et al. (1998), Nicosia and Tomei (1990), Nicosia et al. (1990), Ortega and Spong (1989), Ortega et al. (1998), Slotine et al. (1987), Slotine and $\mathrm{Li}(1987)$ for instance. The main reason that these concepts have not been directly adopted when designing ship control systems are that the hydrodynamic added inertia and damping matrices depend on the wave frequency and the speed of the vessel. The relationship between these quantities and passivity has not been fully understood and therefore neglected in ship control design. In this presentation we will only consider low-speed ship $(U \approx 0)$ applications such as station-keeping and dynamic positioning while cruise control, that is a ship moving at positive constant forward speed $(U=$ constant) will not be covered.

\subsection{Background}

Passivity in ship control was first applied by Sørensen (1993), Sørensen and Egeland (1993). Sørensen and Egeland (1995) used a passive controller to damp out 1st-order wave-induced vibrations in heave for a surface effect ship (SES). In this work, passivity showed to be an excellent tool describing the pressure-induced vibrations of air cushion. This resulted in a set of new design criteria for location of the actuators (fans) and accelerometers in order to obtain perfect collocation.

Motivated by this work the first passive ship autopilot was designed by Paulsen (1996) by treating the ambient water-vessel system as several interconnected massdamper-spring systems. In this work wave filtering and tracking control in 1 degree of freedom (DOF) was addressed under the assumption that there were no wave drift, ocean currents and wind disturbances. A practical design will, however, require integral control in order to compensate for the environmental disturbances. A MIMO extension of this work is found in Paulsen, Egeland and Fossen (1998).

'Department of Engineering Cybernetics, Norwegian University of Science and Technology (NTNU), N-7491 Trondheim, Norway. E-mail: tif@itk.ntnu.no. URL: http://www.itk.ntnu.no/ ansatte/Fossen Thor/. 
More recently, in the period 1998-2000, passivity has been exploited in the design of several commercial ship control systems by Fossen and co-authors. An overview of this work is presented below.

\section{Ship Modeling}

In Section 2, we will present the passivity properties of the ship dynamics. This is based on results of Fossen (1994), Fossen and Fjellstad (1995), and Lewis (1988). It will be distinguished between ships moving at high speed (maneuvering model) and low-speed applications like station-keeping and dynamic positioning. For ships moving at high-speed a shift of frequency due to the incoming waves (frequency of encounter) is observed. Energy conservation of the ship dynamics is treated as two interconnected systems. The first system describes the dissipative motion of the rigid-body (ship) while the second system represents the forces due to potential theory generated by the ambient water particles. It is shown that for a stable ship, both sub-systems are passive as well as the interconnected system. For an unstable ship, e.g. a large tanker, the mapping from velocity to force is input feedforward passive with shortage of passivity. This means that the ship must be stabilized by positive feedback.

\section{Nonlinear Passive Observer Design}

In Section 3 it is shown that for low-speed applications like dynamic positioning (DP) of ships, the structural properties of the ship model can be exploited when designing passive and globally exponentially observers (GES) for reconstruction of velocities, wave frequency motions, and wind and current forces (Fossen and Strand (1999)). In addition, it is possible to adapt the wave frequency on-line (Strand and Fossen (1999)). In existing DP systems, the wave filtering and state estimation problem are solved by using linear Kalman filters (Fossen (1994)). The major drawback of this approach is that the kinematic equations of motions must be linearized about a set of predefined constant yaw angles to cover the whole heading envelope. For each of these linearized models, optimal Kalman filter and feedback control gains are computed (stochastic system with 15 states and 120 covariance equations) such that the gains can be modified on-line by using gain-scheduling techniques. On the contrary, passivity theory showed to be a new tool reducing the number of tuning parameters to a minimum. Experiments with full-scale ships using the passive observer document performance improvements to the Kalman filter.

\section{Nonlinear Passive DP}

In Section 4 the passive observer of Section 3 is used as basis when designing nonlinear DP control systems. Two methods for nonlinear DP is discussed; a DP system derived by using observer backstepping (Fossen and Grøvlen (1998), and Aarset, Strand and Fossen (1998)). Extensions to locally optimal backstepping (LOB) control and globally inverse optimality are done in Strand, Ezal, Fossen and Kokotovic $(1998,1999)$. Finally, a PD-control with bias compensation satisfying a "nonlinear principle" is presented (Loria, Fossen and Panteley (2000)).

\section{Nonlinear Passive Weather Optimal Control}

In Section 5 a new concept for weather optimal positioning control (WOPC) using passivity is discussed (Fossen and Strand (2000)). The main goal of the WOPC is to 
minimize fuel consumption and emission of $\mathrm{CO}_{x} / \mathrm{NO}_{x}$ during station-keeping of ships and floating production vessels offshore. This is done by transforming the ship positioning problem from Cartesian coordinates to polar coordinates. Hence, the ship can be controlled on a circle arc similar to a pendulum in the gravity field. The environmental forces due to wind, waves and currents represent an unknown uniform force field moving the ship to a constant position and weather optimal heading angle. This is obtained by forcing the ship bow to point towards the circle center at the same time as it moves on the circle arc.

\subsection{Problem Formulation}

Based on the models in Section 2 two standard ship control problems are defined. These are both formulated as output feedback control problems since commercial systems are based on position and heading measurements (no velocity measurements). Although there are commercial available velocity and angular velocity sensors, we still have to solve the wave filtering problem. This is usually done by adding two additional states in each DOF of the ship model in order to obtain a notch effect at the dominating wave frequency. Hence, the ship control problems will be output feedback control problems even though all positions and velocities are measured.

In addition to this, the output feedback controller must be implemented with integral action in order to compensate for slowly-varying wave drift forces, wind and ocean currents. A PD-controller cannot be used in a practical implementation. Therefore, the following design requirements must be met when designing a ship control system:

- Output feedback control using position and heading measurements.

- Wave (notch filtering) of position and velocity estimates at the dominating wave frequency.

- Integral action for compensation of wave drift forces, currents and wind loads.

A mathematical formulation for this is given below.

\subsubsection{DOF Low-Speed Control (Dynamic Positioning)}

With low-speed applications we mean station-keeping and marked positioning (tracking control) at speeds $U \approx 0$. This is usually referred to as dynamic positioning (DP). This is a 3 DOF output feedback tracking control problem (surge, sway and yaw) where we can assume that the Coriolis and centripetal forces can be neglected. The resulting model is written, see Section 2 and Sections 3.1 and 3.2 for details:

\begin{tabular}{|ll|}
\hline Kinematics & $\dot{\boldsymbol{\eta}}=\mathbf{J}(\psi) \boldsymbol{v}$ \\
Ship dynamics & $\mathbf{M} \dot{\boldsymbol{v}}+\mathbf{D} \boldsymbol{v}=\boldsymbol{\tau}+\mathbf{J}^{T}(\psi) \mathbf{b}$ \\
2nd-order wave drift + currents + wind & $\dot{\mathbf{b}}=-\mathbf{T}^{-1} \mathbf{b}+\boldsymbol{\Psi} \mathbf{n}$ \\
1st-order wave-induced disturbances & $\dot{\boldsymbol{\xi}}=\mathbf{A}_{\boldsymbol{w}} \boldsymbol{\xi}+\mathbf{E}_{\boldsymbol{w}} \mathbf{w}$ \\
& $\boldsymbol{\eta}_{\boldsymbol{w}}=\mathbf{C}_{\boldsymbol{w}} \xi$ \\
Measurement equation & $\mathbf{y}=\boldsymbol{\eta}+\boldsymbol{\eta}_{\boldsymbol{w}}+\mathbf{v}$ \\
\hline
\end{tabular}




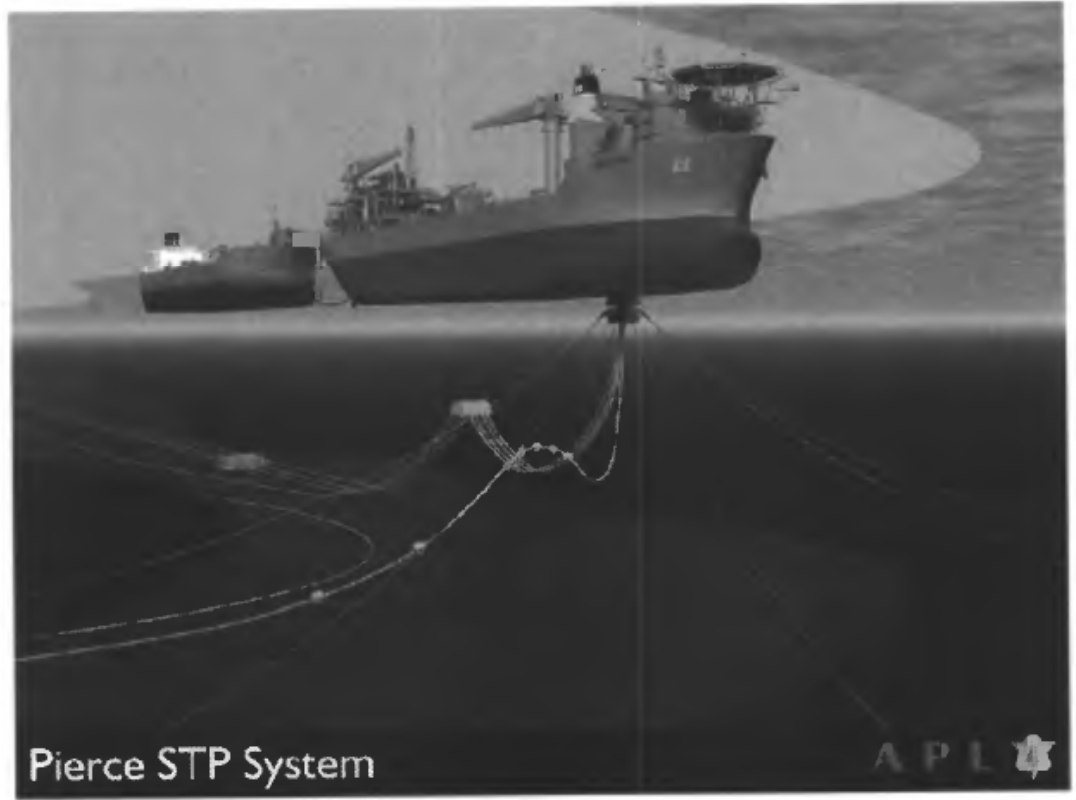

Figure 1. Dynamic positioned ship.

where

$$
\begin{array}{rlrl}
\mathbf{M} & =\mathbf{M}^{T}>0 & \dot{\mathbf{M}}=\mathbf{0} \\
\mathbf{D}=\mathbf{D}^{T}>0 & \dot{\mathbf{D}}=\mathbf{0}
\end{array}
$$

The control objectives are:

$$
\begin{aligned}
& \eta(t) \rightarrow \eta_{d}(t) \\
& v(t) \rightarrow v_{d}(t)
\end{aligned}
$$

where $\boldsymbol{\eta}_{d}, \boldsymbol{v}_{d} \in \mathscr{R}^{3}$ are sufficient smooth reference trajectories. This is an output feedback tracking problem since only $\mathbf{y}$ is available for feedback.

This output feedback control problem has been solved by using observer backstepping (Fossen and Grøvlen (1998), and Aarset, Strand and Fossen (1998)). Extensions to locally optimal backstepping (LOB) control and globally inverse optimality are done in Strand, Ezal, Fossen and Kokotovic (1998, 1999), see also Strand (1999) and references therein.

Another approach is to use PD-control law with bias compensation through a "nonlinear separation principle" (Loria, Fossen and Panteley (2000)).

The nonlinear observer is designed by using SPR-Lyapunov analysis, see Fossen and Strand (1999) and Strand and Fossen (1999).

\subsubsection{DOF Way-Point Tracking Control (Maneuvering)}

Way-point tracking control can be done by using a trajectory generator for generation of a smooth trajectory $\boldsymbol{\eta}_{d} \in \mathscr{R}^{3}$ based on a set of pre-defined way-points. In this case the effect of forward speed $U>0$ must be included in the model matrices. The ship dynamics is written: 


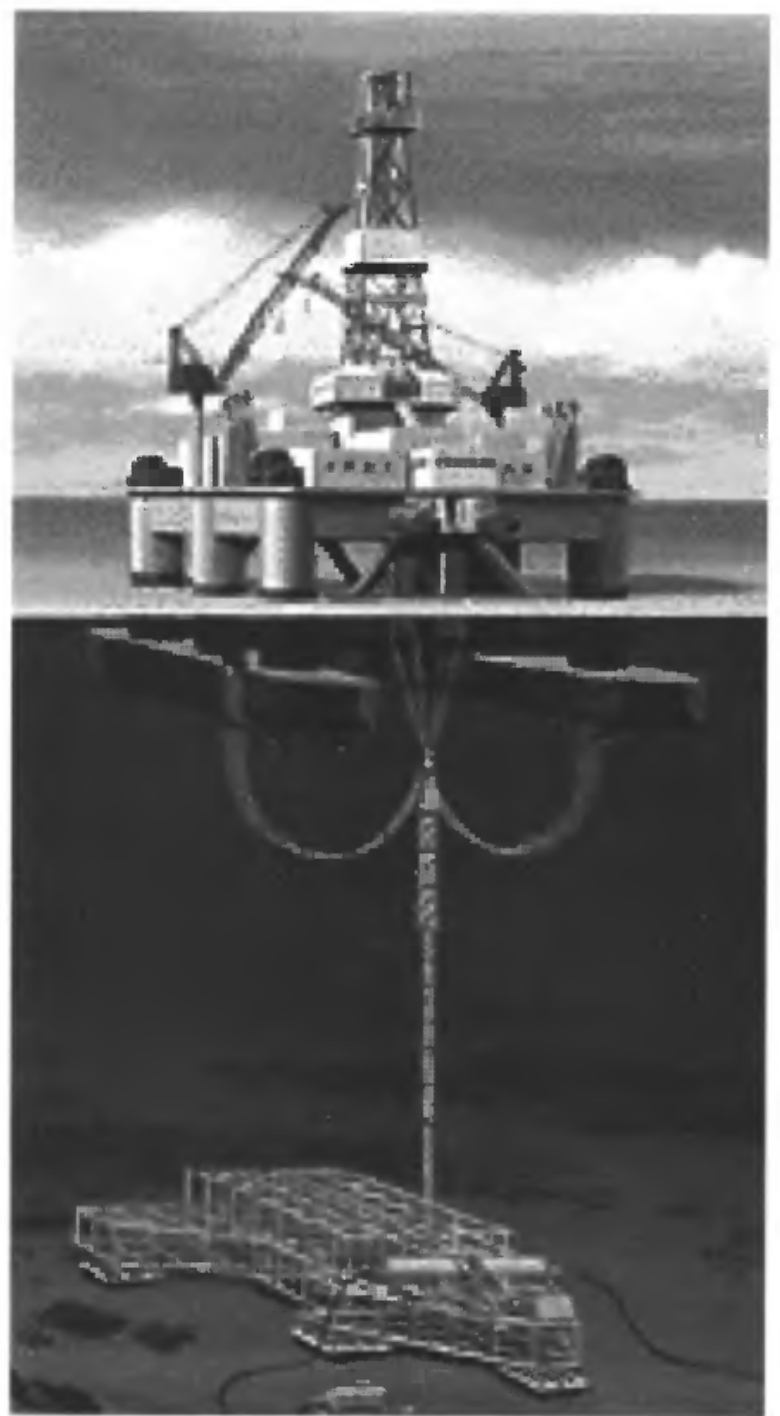

Figure 2. Dynamic positioned semi-submersible.

$$
\mathbf{M}(U) \dot{v}+\mathbf{n}(v)=\tau+\mathbf{J}^{T}(\psi) \mathbf{b}
$$

where $\mathbf{n}(\cdot)$ should include the effect of Coriolis, centripetal and nonlinear damping forces e.g.:

$$
\mathrm{n}(v)=\mathbf{C}(v) v+\mathbf{D}(v) v
$$

The rest in (1) is unchanged while:

$$
\begin{array}{ccc}
\mathbf{M}(U) \neq \mathbf{M}^{T}(U)>0 & \dot{\mathbf{M}}=\mathbf{0} \text { only if } \dot{U}=0 \\
\mathbf{D}(v) \neq \mathbf{D}^{T}(v)>0 & \mathbf{C}(v)=-\mathbf{C}^{T}(v)
\end{array}
$$




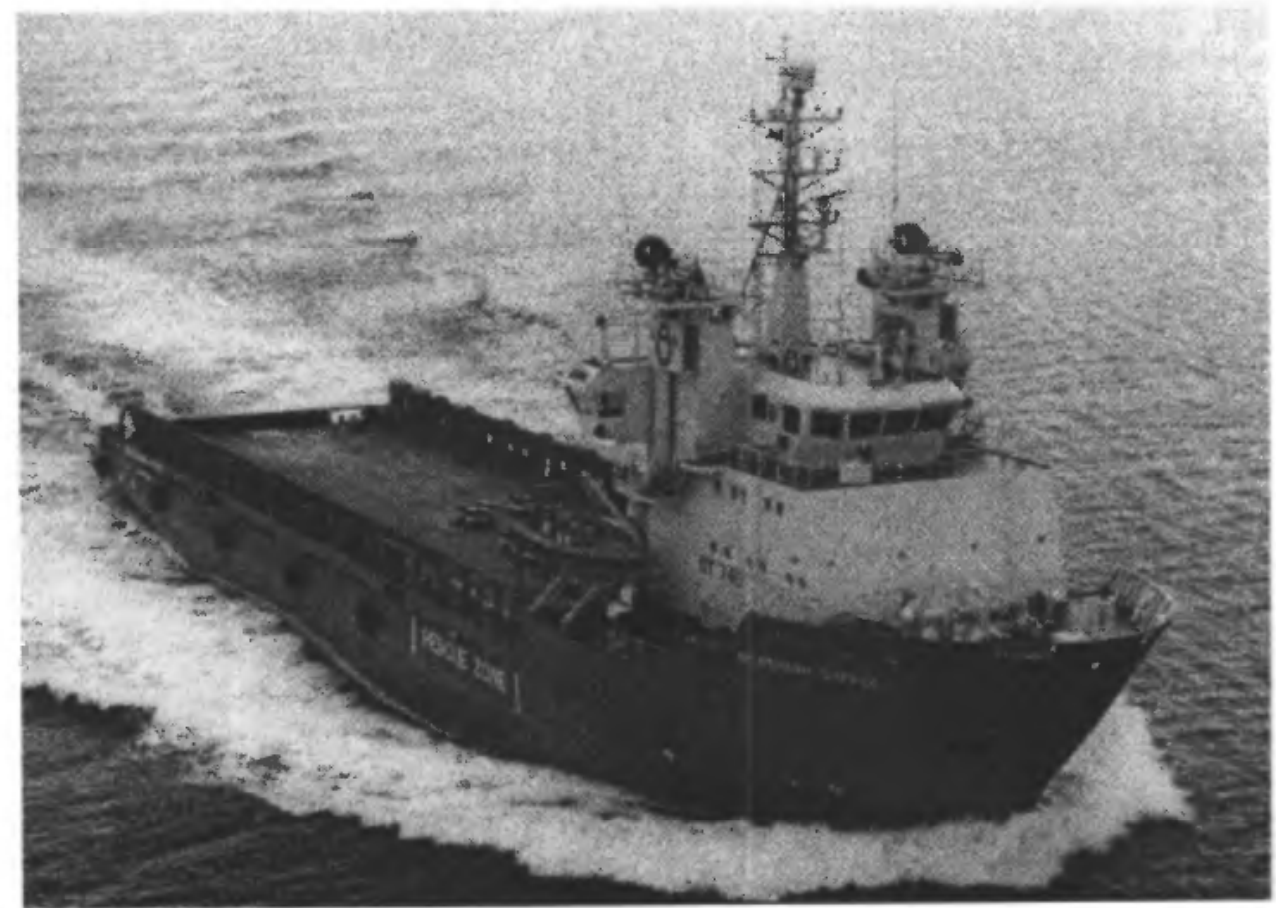

Figure 3. Supply vessel.

The control objective is

$$
\begin{gathered}
\eta(t) \rightarrow \eta_{d}(t) \\
v(t) \rightarrow v_{d}(t)
\end{gathered}
$$

where $\boldsymbol{\eta}_{d}, \boldsymbol{v}_{d} \in \mathscr{R}^{3}$ are sufficient smooth reference trajectories. This is an output feedback tracking problem where a Lyapunov function candidate motivated by kinetic energy of the ambient water-ship system (see Section 2):

$$
\begin{aligned}
V & =\frac{1}{2} v^{T} \mathbf{M}(U) v, \quad \mathbf{M}(U)=\mathbf{M}_{R B}+\mathbf{M}_{A}(U) \\
& =\frac{1}{2} v^{T}\left(\mathbf{M}_{R B}+\mathbf{M}_{A}(U)\right) v \\
& =\frac{1}{2} v^{T}\left(\mathbf{M}_{R B}+\frac{1}{2}\left(\mathbf{M}_{A}(U)+\mathbf{M}_{A}^{T}(U)\right)\right) v \\
\dot{V} & =v^{T}\left(\mathbf{M}_{R B}+\frac{1}{2}\left(\mathbf{M}_{A}(U)+\mathbf{M}_{A}^{T}(U)\right)\right) \dot{v}+\frac{1}{2} v^{T} \dot{M}_{A}(U) v \\
& =v^{T} \mathbf{M}(U) \dot{v}+\frac{1}{2} v^{T}\left(\dot{M}_{A}(U)-\mathbf{M}_{A}(U)+\mathbf{M}_{A}^{T}(U)\right) \dot{v}
\end{aligned}
$$

fails due to the non-symmetry of $\mathbf{M}_{A}(U)$. Hence, it is not clear what we should do with the second term in the expression for $\dot{V}$. However, the Lyapunov function candidate $V=(1 / 2) v^{T} v$ and $\dot{V}=v^{T} \dot{v}$ can be used instead since $\mathbf{M}(U)$ is invertible for all $U$. Moreover: 


$$
\dot{v}=\mathbf{M}^{-1}(U)\left[\tau+\mathbf{J}^{T}(\psi) \mathbf{b}-\mathbf{n}(v, U)\right]
$$

This also means that feedback linearization works, see Berge (1999) for examples.

\section{Nonlinear Modeling of Ships}

In this section we will give a brief introduction to ship modeling.

\subsection{Ship Equations of Motion}

For a conventional ship it is common to consider the motion in surge (forward), sway (sideways) and yaw (heading), see Figure 4. Denote the control forces and moments in surge, sway and yaw by $\tau=\left[\tau_{1}, \tau_{2}, \tau_{3}\right]^{T}$, the velocity vector decomposed in a body-fixed frame as $v=[u, v, r]^{T}$ and the Earth-fixed positions/Euler-angles as $\boldsymbol{\eta}=[x, y, \psi]^{T}$.

Then the nonlinear ship model in 3 DOF (surge, sway and yaw) can be written, see Fossen (1994):

$$
\begin{gathered}
\mathbf{M}(U) \dot{\boldsymbol{v}}+\mathbf{n}(\boldsymbol{v})=\boldsymbol{\tau}+\mathbf{J}^{T}(\psi) \mathbf{b} \\
\dot{\boldsymbol{\eta}}=\mathbf{J}(\psi) \boldsymbol{v}
\end{gathered}
$$

where $U=\sqrt{u^{2}+v^{2}}$ is the forward speed, $\mathbf{b} \in \mathscr{R}^{3}$ is slowly-varying bias term due to wind, currents and wave drift forces, $M \in \mathscr{R}^{3 \times 3}$ is the speed dependent inertia matrix, $\mathbf{n}(\cdot) \in \mathscr{R}^{3}$ is a nonlinear function of Coriolis, centripetal and damping forces and $\tau \in \mathscr{R}^{3}$ is a vector of control forces and moment.

$\mathbf{J}(a): \mathbb{R} \rightarrow S O(3)$ is the rotation matrix in yaw given by:

$$
\mathbf{J}(a)=\left[\begin{array}{ccc}
\cos a & -\sin a & 0 \\
\sin a & \cos a & 0 \\
0 & 0 & 1
\end{array}\right]
$$

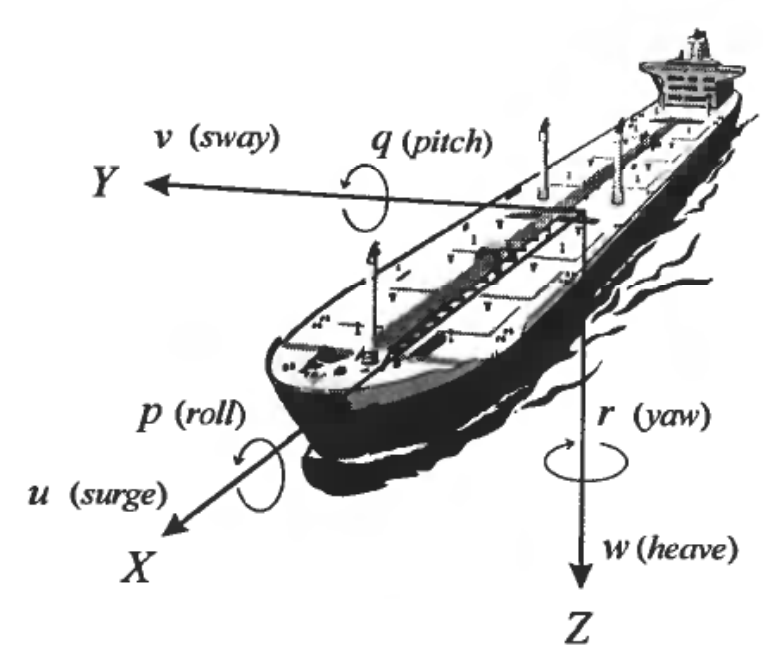

Figure 4. Definition of state variables in surge, sway, heave, roll, pitch and yaw for a marine vessel. 
Notice that $\mathbf{J}^{-1}(a)=\mathbf{J}^{T}(a)$. Let $\mathbf{C}$ be a matrix of Coriolis and centripetal terms and $\mathbf{D}$ be a matrix of damping terms. Hence, for a ship in $3 \mathrm{DOF}$ the nonlinear terms in the ship model can be collected into a vector given by:

$$
\mathrm{n}(v)=\mathbf{C}(v) v+\mathbf{D}(v) v
$$

The different matrices can be represented as:

$$
\begin{aligned}
\mathbf{M}(U) & =\mathbf{M}_{R B}+\mathbf{M}_{A}(U) \\
\mathbf{C}(v) & =\mathbf{C}_{R B}(v)+\mathbf{C}_{A}(v) \\
\mathbf{D}(v) & =\mathbf{D}_{L}+\mathbf{D}_{N}(v)
\end{aligned}
$$

Here the subscripts denote $R B$ (rigid-body) and $A$ (hydrodynamic added mass). The damping matrix $\mathbf{D}(v)$ is portioned into a linear part $\mathbf{D}_{L}$ and a nonlinear part $\mathbf{D}_{N}(v)$. Several hydrodynamic effects contributes to the damping matrix. These include potential damping, damping due to Morrison's equation (vortex shedding), wave drift damping, skin friction etc., see Fossen (1994) for more details.

For 3 DOF the inertia matrix takes the form:

$$
\mathbf{M}_{R B}=\mathbf{M}_{R B}^{T}=\left[\begin{array}{ccc}
m & 0 & 0 \\
0 & m & m x_{G} \\
0 & m x_{G} & I_{z}
\end{array}\right]>0
$$

where $m$ is the mass, $x_{G}$ is the x-coordinate to the center of gravity and $I_{z}=I_{C G}+m x_{G}^{2}$ is the moment of inertia. The hydrodynamic added inertia matrix $\mathbf{M}_{A}$ is in general speed dependent and non-symmetrical, that is:

$$
\mathbf{M}_{A}(U)=\left[\begin{array}{ccc}
-X_{\dot{u}} & 0 & 0 \\
0 & -Y_{\dot{v}} & -Y_{\dot{r}} \\
0 & -N_{\dot{v}} & -N_{\dot{r}}
\end{array}\right]>0
$$

where the hydrodynamic coefficients $X_{\dot{u}}, Y_{\dot{v}}, Y_{\dot{r}}, N_{\dot{v}}$ and $N_{\dot{r}}$ depend on the forward speed $U$. For low-speed applications $(U \approx 0)$ under the assumption of no incoming waves $\mathbf{M}_{A}=\mathbf{M}_{A}^{T}$, that is $Y_{\dot{r}}=N_{\dot{v}}$. Similarly, linear damping is written as:

$$
\mathbf{D}_{L}=\left[\begin{array}{ccc}
-X_{u} & 0 & 0 \\
0 & -Y_{v} & -Y_{r} \\
0 & -N_{v} & -N_{r}
\end{array}\right]
$$

At low speed under the assumption of no incoming waves it can be shown that $\mathbf{D}_{L}=\mathbf{D}_{L}^{T}$. The Coriolis and centripetal matrix:

$$
\mathrm{C}(v)=\mathrm{C}_{R B}(v)+\mathrm{C}_{A}(v)
$$

is given by (Fossen (1994)): 


$$
\begin{gathered}
\mathbf{C}_{R B}(v)=-\mathbf{C}_{R B}^{T}(v)=\left[\begin{array}{ccc}
0 & 0 & -m\left(x_{G} r+v\right) \\
0 & 0 & m u \\
m\left(x_{G} r+v\right) & -m u & 0
\end{array}\right] \\
\mathbf{C}_{A}(v)=-\mathbf{C}_{A}^{T}(v)=\left[\begin{array}{ccc}
0 & 0 & Y_{\dot{v}} v+Y_{\dot{r}} r \\
0 & 0 & -X_{\dot{u}} u \\
-Y_{\dot{v}} v-Y_{\dot{r}} r & X_{\dot{u}} u & 0
\end{array}\right]
\end{gathered}
$$

\subsection{Nonlinear Low-Speed Model}

In dynamic positioning (DP) systems a low-speed model is used for station-keeping. Moreover:

$$
U \approx 0
$$

implies that (11), (12) and (14) can be approximated by:

$$
\begin{gathered}
\mathbf{M} \dot{v}+\mathbf{D} \boldsymbol{v}=\boldsymbol{\tau}+\mathbf{J}^{T}(\psi) \mathbf{b} \\
\dot{\eta}=\mathbf{J}(\psi) v
\end{gathered}
$$

where

$$
\begin{aligned}
\mathbf{M} & =\mathbf{M}^{T}=\left[\begin{array}{ccc}
m-X_{\dot{u}} & 0 & 0 \\
0 & m-Y_{\dot{v}} & m x_{G}-Y_{\dot{r}} \\
0 & m x_{G}-Y_{\dot{r}} & I_{z}-N_{\dot{r}}
\end{array}\right]>0 \\
\mathbf{D} & =\mathbf{D}^{T}=\left[\begin{array}{ccc}
-X_{u} & 0 & 0 \\
0 & -Y_{v} & -Y_{r} \\
0 & -Y_{r} & -N_{r}
\end{array}\right]>0
\end{aligned}
$$

\subsection{Nonlinear Model Properties}

Based on the nonlinear model above several definitions and theorems will now be stated in order to exploit the properties of the proposed model structure.

\subsubsection{Properties of the Inertia Matrix}

The inertia matrix $\mathbf{M}$ is written as:

$$
\mathbf{M}=\mathbf{M}_{R B}+\mathbf{M}_{\boldsymbol{A}}
$$

where $\mathbf{M}_{R B}$ is the rigid-body inertia matrix and $\mathbf{M}_{A}$ is the hydrodynamic added inertia matrix. For these matrices the following properties hold:

P1 $\mathbf{M}_{R B}=\mathbf{M}_{R B}^{T}$ is a positive definite matrix.

$\mathrm{P} 2 \mathrm{M}_{A}$ is a strictly positive matrix for $U>0$ (positive definite at low speed $U \approx 0$ ). 
Theorem 2.1 (Intertia Matrix) The inertia matrix $\mathrm{M}$ is strictly positive, i.e. $v^{T} \mathbf{M} v>0 \forall v \neq \mathbf{0}$.

Proof. This follows directly from Properties P1 and P2:

$$
v^{T} \mathbf{M} v=v^{T} \mathbf{M}_{R B} v+\frac{1}{2} v^{T}\left(M_{A}+\mathbf{M}_{A}^{T}\right) v>0, \forall v \neq 0
$$

\subsubsection{Properties of the Coriolis and Centripetal Matrix}

Consider the Coriolis and centripetal matrix:

$$
C(v)=C_{R B}(v)+C_{A}(v)
$$

Theorem 2.2 (Coriolis and Centripetal Matrix) The matrix $\mathbf{C}(v)$ is skew-symmetrical, that is

$$
\mathbf{C}(v)=-\mathbf{C}^{T}(v)
$$

Proof. See Fossen and Fjellstad (1995).

\subsubsection{Properties of the Damping Matrix}

Consider the damping matrix:

$$
\mathbf{D}(v)=\mathbf{D}_{L}+\mathbf{D}_{N}(v)
$$

where:

1. $\mathbf{D}_{L}>0$ for a straight-line stable ship.

2. $\mathbf{D}_{L} \leqslant 0$ for a marginally or unstable ship.

Theorem 2.3 (Nonlinear Damping Matrix) The damping matrix $\mathbf{D}_{N}(v)$ is strictly positive, i.e. $v^{T} \mathbf{D}_{N}(v) v \geqslant 0 \forall v \neq \mathbf{0}$.

Proof. This follows directly from the dissipative nature of the damping forces.

\subsection{Dissipativity and Passivity Properties}

The dissipativity and passivity properties of the ship are of interest when designing ship control systems using the energy conservation. A discussion on passive and dissipative control design is found in Hill and Moylan (1977). The main idea is that every nonlinear passive system which is controlled by a passive controller is closed loop-stable. Hence, the passivity properties of the ship model should be investigated before designing a passive controller. The general framework and definitions below are adopted from Willems (1972) and Sepulchre et al. (1997).

Consider the ship as two separate systems which are interconnected by each other 


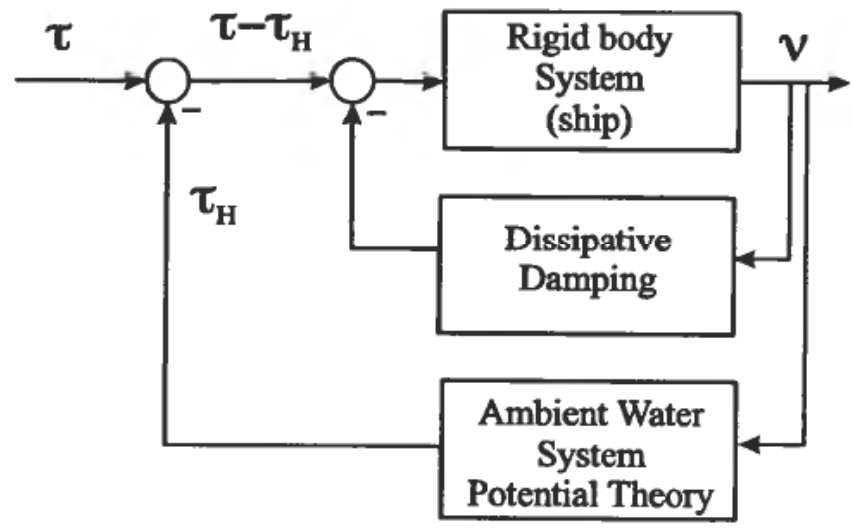

Figure 5. The upper system describes the motion of the rigid body (ship) including dissipative damping. The lower system represents the forces due to potential theory generated by the ambient water particles.

as shown in Figure 5. The first system describes the dissipative motion of the rigid body and it is passive in the sense that all dissipative forces are passive. The second system describes the forces generated from the ambient water particles due to potential theory (forced body oscillations) for a ship moving at constant speed.

Under the assumption of low speed the nonlinear models (11)-(12) can be written as:

$$
\begin{gathered}
\mathbf{M}_{R B} \dot{v}+\mathbf{C}_{R B}(v) v+\mathrm{D}_{N}(v) v=\tau-\tau_{H} \\
\mathbf{M}_{A} \dot{v}+\mathrm{C}_{A}(v) v+\mathrm{D}_{L} v=\tau_{H}
\end{gathered}
$$

where $\tau$ is the external forces due to thrusters, wind, currents and waves and $\tau_{H}$ is the hydrodynamic forces and moments due to forced oscillations including linear potential and viscous damping.

Theorem 2.4 (Passive Rigid-Body Dynamics) The rigid-body dynamics: $\mathbf{M}_{R B} \dot{v}_{+} \mathbf{C}_{R B}(v) v$ including nonlinear dissipative damping $\mathbf{D}_{N}(v) v$ is passive, i.e. the mapping $\left(\tau-\tau_{H}\right)$ to $v$ is passive.

Proof. Consider the storage function (kinetic energy):

$$
V_{1}=\frac{1}{2} v^{T} \mathbf{M}_{R B} v
$$

which is a positive definite function in $v$, then the integral:

$$
\begin{aligned}
\int_{0}^{T} v^{T}\left(\tau-\tau_{H}\right) d t & =\int_{0}^{T} v^{T}\left(\mathbf{M}_{R B} \dot{v}+\mathbf{C}_{R B}(v) v+\mathbf{D}_{N}(v) v\right) d t \\
& =\int_{0}^{T} v^{T} \mathbf{M}_{R B} \dot{v} d t+\int_{0}^{T} v^{T} \mathbf{D}_{N}(v) v d t \\
& =V_{1}(T)-V_{1}(0)+\int_{0}^{T} v^{T} \mathbf{D}_{N}(v) v d t
\end{aligned}
$$


We have here used the properties $\boldsymbol{v}^{T} \mathbf{C}_{R B}(v) v=0, \mathbf{M}_{R B}=\mathbf{M}_{R B}^{T}>0$ and $\dot{\mathbf{M}}_{R B}=\mathbf{0}$. Furthermore, since viscous damping forces are dissipative i.e.: $\int_{0}^{T} v^{T} \mathbf{D}_{N}(v) v d t>0$, then:

$$
V_{1}(T)-V_{1}(0)<\int_{0}^{T} v^{T}\left(\tau-\tau_{H}\right) d t
$$

which completes the proof.

Theorem 2.5 (Passive Dynamics of the Ambient Water Particles) The dynamics of the ambient water particles is passive from $v$ to $\tau_{H}$.

Proof. Consider the storage function (kinetic energy due to hydrodynamic added inertia):

$$
V_{2}=\frac{1}{2} v^{T} \mathbf{M}_{A} v
$$

which is a positive definite function in $v$, then the integral:

$$
\begin{aligned}
\int_{0}^{T} v^{T} \tau_{H} d t & =\int_{0}^{T} v^{T}\left(\mathbf{M}_{A} \dot{v}+\mathbf{C}_{A}(v) v+\mathbf{D}_{L} v\right) d t \\
& =\int_{0}^{T} v^{T} \mathbf{M}_{A} \dot{v} d t+\int_{0}^{T} v^{T} \mathbf{D}_{L} v d t \\
& =V_{2}(T)-V_{2}(0)+\int_{0}^{T} v^{T} \mathbf{D}_{L} v d t
\end{aligned}
$$

We have here used the property that: $v^{T} \mathbf{C}_{A}(v) v=0$ and that the integral of $v^{T} \mathbf{M}_{A} \dot{v}$ where $\mathbf{M}_{A} \neq \mathbf{M}_{A}^{T}$ can be written:

$$
\begin{aligned}
\int_{0}^{T} v^{T} \mathbf{M}_{A} \dot{v} d t & =\int_{0}^{T} v^{T} \mathbf{M}_{A} d v \\
& =\left.\frac{1}{2} v^{T} \mathbf{M}_{A} v\right|_{0} ^{T}-\frac{1}{2} \int_{0}^{T} v^{T} \dot{M}_{A} v d t
\end{aligned}
$$

For low-speed applications $\dot{\mathbf{M}}_{A}=\mathbf{0}$. Hence:

$$
\int_{0}^{T} v^{T} \mathbf{M}_{A} \dot{v} d t=\left.\frac{1}{2} v^{T} \mathbf{M}_{A} v\right|_{0} ^{T}=V_{2}(T)-V_{2}(0)
$$

Hence, the following considerations can be made:

1. For a stable ship, the mapping from $v$ to $\tau_{H}$ is passive.

Since: $\int_{0}^{T} \boldsymbol{v}^{T} \mathbf{D}_{L} v d t \geqslant 0$, we have:

$$
V_{2}(T)-V_{2}(0) \leqslant \int_{0}^{T} v^{T} \tau_{H} d t
$$


2. For an unstable ship, the mapping from $v$ to $\tau_{H}$ is input feedforward passive. Let $\min \left(v^{T} \mathbf{D}_{L} v\right)=\lambda_{\min }\left(\mathbf{D}_{L}\right) v^{T} v$, where $\lambda_{\min }\left(\mathbf{D}_{L}\right) \leqslant 0$ is the smallest eigenvalue of the matrix $\mathbf{D}_{L}$. Then

$$
\int_{0}^{T} v^{T} \mathbf{D}_{L} v d t \geqslant \lambda_{\min }\left(\mathbf{D}_{L}\right) \int_{0}^{T} v^{T} v d t
$$

and hence:

$$
V_{2}(T)-V_{2}(0) \leqslant \int_{0}^{T}\left(v^{T} \tau_{H}-\lambda_{\min }\left(\mathrm{D}_{L}\right) v^{T} v\right) d t
$$

This means that the system is input feedforward passive which is seen by choosing the storage function as $w\left(v, \tau_{H}\right)=v^{T} \tau_{H}-\lambda_{\min }\left(\mathbf{D}_{L}\right) v^{T} v$. Since $\lambda_{\text {min }}\left(\mathbf{D}_{L}\right) \leqslant 0$, there is a shortage of passivity according to the definition made by Sepulchre et al. (1997), i.e. the system must be stabilized with positive feedback to achieve passivity. This is typically for unstable tankers.

The result above states that for any stable ship, the mapping from $\tau$ to $v$ is passive. This is due to the properties of two passive interconnected systems. In the case of an unstable ship, the mapping from $\tau$ to $v$ is not passive and the ship must be stabilized by positive feedback to obtain a stable closed-loop system.

\section{Nonlinear Passive Observer Design}

Filtering and state estimation are important features of a DP system. In most cases, measurements of the vessel velocities are not available. Hence, estimates of the velocities must be computed from noisy position and heading measurements through a state observer. Unfortunately, the position and heading measurements are corrupted with colored noise due to wind, waves and ocean currents as well as sensor noise. However, only the slowly-varying disturbances should be counteracted by the propulsion system, whereas the oscillatory motion due to the waves (1st-order wave disturbances) should not enter the feedback loop. This is done by using so-called wave filtering techniques, which separates the position and heading measurements into a low-frequency (LF) and wave frequency (WF) position and heading estimate (Fossen (1994)).

In existing DP systems the wave filtering and state estimation problem are solved by using linear Kalman filters. The major drawback of this approach is that the kinematic equations of motions must be linearized about a set of predefined constant yaw angles, typically 36 operating points in steps of 10 degrees, to cover the whole heading envelope. For each of these linearized models, optimal Kalman filter and feedback control gains are computed such that the gains can be modified on-line by using gain-scheduling techniques. In the seek for new control strategies the linear Kalman filter approach is a major obstacle since it is difficult and time-consuming to tune the state estimator (stochastic system with 15 states and 120 covariance equations). The main reason for this, is that the numerous covariance tuning parameters may be difficult to relate to physical quantities resulting in a somewhat ad hoc tuning procedure.

In this section the nonlinear passive observer of Fossen and Strand (1999) is presented, see Nijmeijer and Fossen (1999) for a more general discussion on nonlinear observer design. The passive observer includes wave filtering properties, bias state 
estimation, reconstruction of the LF motion components and noise-free estimates of the non-measured vessel velocities. The proposed observer is proven to be passive and GES. Hence, only one set of observer gains are needed to cover the whole state space. In addition, the number of observer tuning parameters are significantly reduced and the wave filter parameters are directly coupled to the dominating wave frequency. Passivity theory showed to be a new tool with respect to accurate tuning of the observer.

\subsection{DP Ship Model}

The low-frequency motion of a large class of surface ships can be described by the following model:

$$
\begin{aligned}
\mathbf{M} \dot{v}+\mathbf{D} v & =\tau+\mathbf{J}^{T}(\psi) \mathbf{b} \\
\dot{\mathbf{b}} & =-\mathbf{T}^{-\mathbf{1}} \mathbf{b}+\boldsymbol{\Psi} \mathbf{n} \\
\dot{\boldsymbol{\eta}} & =\mathbf{J}(\psi) \boldsymbol{v}
\end{aligned}
$$

where external forces and moment due to slowly-varying wind, currents and waves are lumped together into an Earth-fixed bias term $\mathbf{b} \in \mathscr{R}^{3}$.

\subsection{1st-Order Wave-Induced Model}

A linear 2nd-order wave frequency (WF) model is considered to be sufficient for representing the WF-induced motions and can be formulated as

$$
\begin{aligned}
\dot{\xi} & =\mathbf{A}_{w} \xi+\mathbf{E}_{w} \mathbf{w} \\
\boldsymbol{\eta}_{w} & =\mathbf{C}_{w} \xi
\end{aligned}
$$

where $\boldsymbol{\eta}_{w}=\left[x_{w}, y_{w}, \psi_{w}\right]^{T}, \xi \in \mathbb{R}^{6}$ and $\mathbf{w}_{w}=\left[w_{1}, w_{2}, w_{3}\right]^{T} \in \mathbb{R}^{3}$ is a zero-mean Gaussian white noise vector and:

$$
\mathbf{A}_{w}=\left[\begin{array}{cc}
\mathbf{0} & \mathbf{I} \\
-\mathbf{\Omega}^{2} & -2 \Lambda \Omega
\end{array}\right], \quad \mathbf{C}_{\boldsymbol{w}}=\left[\begin{array}{ll}
\mathbf{0} & \mathbf{I}
\end{array}\right], \quad \mathbf{E}_{w}=\left[\begin{array}{l}
\mathbf{0} \\
\mathbf{K}_{w}
\end{array}\right]
$$

where $\Omega=\operatorname{diag}\left\{\omega_{1}, \omega_{2}, \omega_{3}\right\}, \Lambda=\operatorname{diag}\left\{\zeta_{1}, \zeta_{2}, \zeta_{3}\right\}$ and $\mathbf{K}_{w}=\operatorname{diag}\left\{K_{w 1}, K_{w 2}, K_{w 3}\right\}$. This model corresponds to three decoupled WF models:

$$
\frac{\eta_{w_{i}}}{w_{i}}(s)=\frac{K_{w_{i}} s}{s^{2}+2 \zeta_{i} \omega_{i} s+\omega_{i}^{2}}, \quad(i=1,2,3)
$$

where $\omega_{i}(i=1 \ldots 3)$ are the dominating wave frequencies, $\zeta_{i}(i=1 \ldots 3)$ are the relative damping ratios and $K_{w_{i}}(i=1 \ldots 3)$ are parameters related to the wave intensity representing the 1st-order wave-induced disturbances on the vessel. From a practical point of view, the WF model parameters are slowly-varying quantities, depending on the prevailing sea state. Typically, the periods $T_{i}$ of the dominating waves are in the range of 5 to 20 seconds in the North Sea corresponding to a wave frequency $\omega_{i}=2 \pi / T_{i}$. The relative damping ratios $\zeta_{i}$ will typically be in the range $0.05-0.10$.

\subsubsection{Wave Filtering}

The WF response of the ship is generated by using the principle of linear superposition, that is the 1 st-order wave-induced motion $\eta_{w}$ is added to the LF motion 


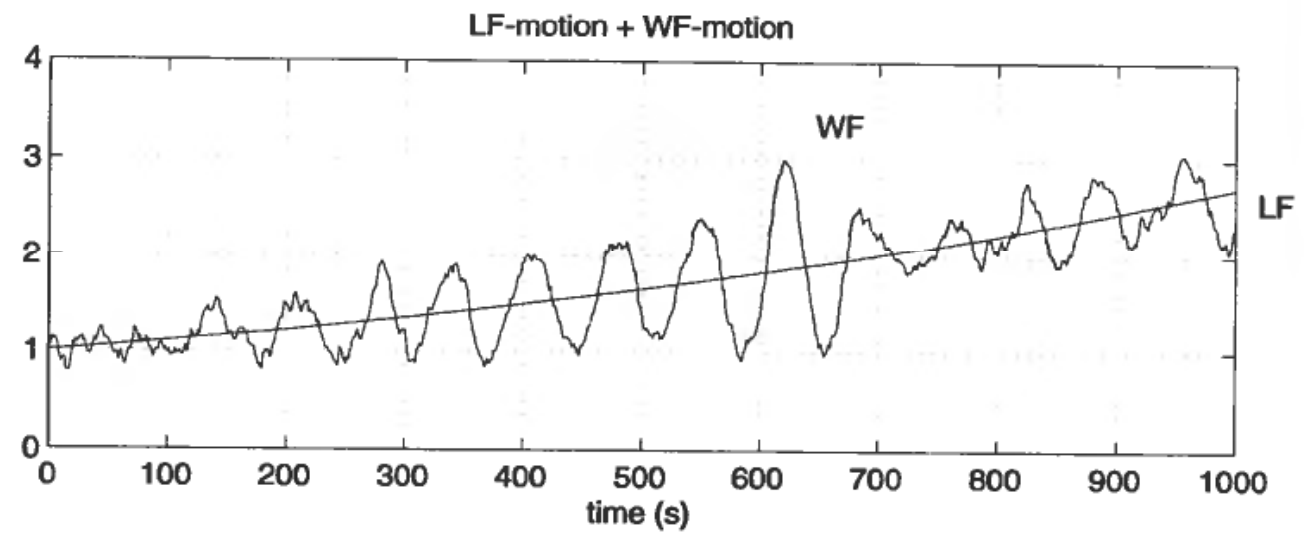

Figure 6. Figure showing the total ship motion as the sum of the LF-motion and the WFmotion.

components of the ship given by $\eta$. Hence, the total ship motion is the sum of the LFmotion components and the WF-motion components as shown in Figure 6, that is:

$$
\mathbf{y}=\boldsymbol{\eta}+\boldsymbol{\eta}_{w}+\mathbf{v}
$$

where $\mathbf{v} \in \mathscr{R}^{3}$ is white Gaussian measurement noise. Notice the oscillatory behavior of the wave-induced motion component. It is also assumed that the WF model excitation $\mathbf{w} \in \mathscr{R}^{3}$ in (43) is zero-mean Gaussian white noise.

The main objective of the observer is to perform wave filtering:

Definition 3.1 (Wave Filtering) Wave Filtering can be defined as the reconstruction of the LF motion components $\boldsymbol{\eta}$ from the noisy measurement $\mathbf{y}=\boldsymbol{\eta}+\boldsymbol{\eta}_{\boldsymbol{w}}+\mathbf{v}$ by means of an observer (state estimator). In addition to this, a noise-free estimate of the LF velocity $v$ should be produced from $\mathbf{y}$. This is crucial in ship motion control systems since the oscillatory motion $\eta_{w}$ due to 1st-order wave-induced disturbances will, if it enters the feedback loop, cause wear and tear of the actuators and increase the fuel consumption.

Remark 3.1 In general it is impossible to counteract the 1st-order wave-induced motion of a ship when applying a reasonable propulsion and thruster system. Hence, no improvement in position performance should be expected by feeding back the signal $\boldsymbol{\eta}_{w}$ to the controller.

\subsection{Nonlinear Observer Design}

When designing the observer we will assume that $\mathbf{J}(\psi) \approx \mathbf{J}\left(\psi+\psi_{w}\right)$. This is a good assumption since the magnitude of the wave-induced yaw disturbance $\psi_{w}$ will be less than 5 degrees in extreme weather situations (sea state codes 5-10), and less than 1 degree during normal operation of the ship (sea state codes 1-5). We will also neglect the zero-mean white noise terms $\mathbf{n}, \mathbf{w}$ and $\mathbf{v}$ in (42), (43) and (46) in the Lyapunov analysis (deterministic approach). The Lyapunov stability analysis is only used to derive the nonlinear observer structure. When implementing the observer, this assumption can be relaxed. This implies that the observer error states will converge to balls 
proportional with the covariances of the white noise signals $\mathbf{n}, \mathbf{w}$ and $\mathbf{v}$ instead of the origin itself.

Based on this the observer model takes the form:

$$
\begin{aligned}
\dot{\boldsymbol{\xi}} & =\mathbf{A}_{w} \boldsymbol{\xi} \\
\dot{\boldsymbol{\eta}} & =\mathbf{J}\left(\psi_{y}\right) \boldsymbol{v} \\
\dot{\mathbf{b}} & =-\mathbf{T}^{-1} \mathbf{b} \\
\mathbf{M} \dot{\boldsymbol{v}} & =-\mathbf{D} \boldsymbol{v}+\mathbf{J}^{T}\left(\psi_{y}\right) \mathbf{b}+\tau \\
\mathbf{y} & =\boldsymbol{\eta}+\boldsymbol{\eta}_{\boldsymbol{w}}=\boldsymbol{\eta}+\mathbf{C}_{\boldsymbol{w}} \xi
\end{aligned}
$$

where

$$
\psi_{y}=\psi+\psi_{w}
$$

is the compass measurement. For notational simplicity the states $\xi, \eta$ and the measurement $\mathbf{y}$ are written in state-space form:

$$
\begin{aligned}
\dot{\boldsymbol{\eta}}_{0} & =\mathbf{A}_{0} \boldsymbol{\eta}_{0}+\mathbf{B}_{0} \mathbf{J}\left(\psi_{y}\right) \boldsymbol{v} \\
\mathbf{y} & =\mathbf{C}_{0} \boldsymbol{\eta}_{0}
\end{aligned}
$$

where $\eta_{0}=\left[\xi^{T}, \eta^{T}\right]^{T}$ and:

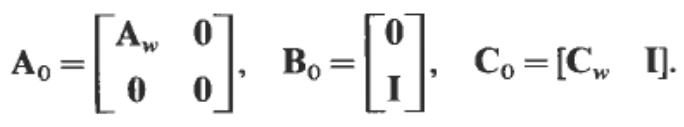

A nonlinear observer copying the dynamics (47) is:

$$
\begin{aligned}
\dot{\hat{\xi}} & =\mathbf{A}_{w} \hat{\xi}+\mathbf{K}_{1} \tilde{\mathbf{y}} \\
\dot{\hat{\boldsymbol{\eta}}} & =\mathbf{J}\left(\psi_{y}\right) \hat{\mathbf{v}}+\mathbf{K}_{2} \tilde{\mathbf{y}} \\
\dot{\hat{\mathbf{b}}} & =-\mathbf{T}^{-1} \hat{\mathbf{b}}+\frac{1}{\gamma} \mathbf{K}_{3} \tilde{\mathbf{y}} \\
\mathbf{M} \dot{\hat{\mathbf{v}}} & =-\mathbf{D} \hat{\boldsymbol{v}}+\mathbf{J}^{T}\left(\psi_{y}\right) \hat{\mathbf{b}}+\tau+\frac{1}{\gamma} \mathbf{J}^{T}\left(\psi_{y}\right) \mathbf{K}_{4} \tilde{\mathbf{y}} \\
\hat{\mathbf{y}} & =\hat{\boldsymbol{\eta}}+\mathbf{C}_{\boldsymbol{w}} \hat{\xi}
\end{aligned}
$$

where $\tilde{\mathbf{y}}=\mathbf{y}-\hat{\mathbf{y}}$ is the estimation error and $\mathbf{K}_{1} \in \mathscr{R}^{6 \times 3}, \mathbf{K}_{2}, \mathbf{K}_{3}, \mathbf{K}_{4} \in \mathscr{R}^{3 \times 3}$ are observer gain matrices to be interpreted later. $\gamma>0$ is an additional scalar tuning parameter motivated by the Lyapunov analysis. Similarly as (49), the system (51) is written in state-space form:

$$
\begin{aligned}
\dot{\hat{\boldsymbol{\eta}}}_{0} & =\mathbf{A}_{0} \hat{\boldsymbol{\eta}}_{0}+\mathbf{B}_{0} \mathbf{J}\left(\psi_{y}\right) \hat{\boldsymbol{v}}+\mathbf{K} \tilde{\mathbf{y}} \\
\hat{\mathbf{y}} & =\mathbf{C}_{\mathbf{0}} \hat{\boldsymbol{\eta}}_{0}
\end{aligned}
$$




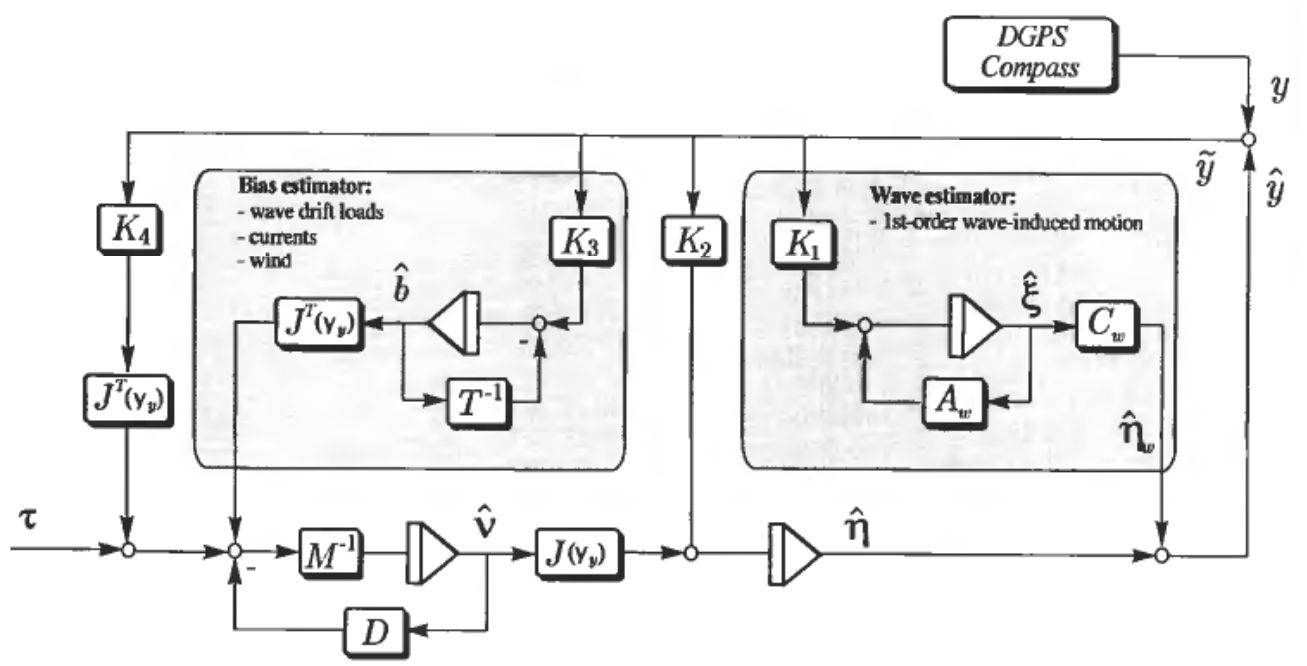

Figure 7. Block diagram showing the nonlinear observer.

where $\hat{\eta}_{0}=\left[\hat{\xi}^{T}, \hat{\eta}^{T}\right]^{T}$ and:

$$
K=\left[\begin{array}{l}
K_{1} \\
K_{2}
\end{array}\right]
$$

The observer structure is shown in Figure 7.

\subsection{Determination of the Observer Gain Matrices}

The estimation errors are defined as $\tilde{\boldsymbol{v}}=\boldsymbol{v}-\hat{\boldsymbol{v}}, \tilde{\mathbf{b}}=\mathbf{b}-\hat{\mathbf{b}}$ and $\tilde{\boldsymbol{\eta}}_{0}=\boldsymbol{\eta}_{0}-\hat{\boldsymbol{\eta}}_{0}$. Hence, the error dynamics can be written:

$$
\begin{aligned}
\dot{\tilde{\eta}}_{0} & =\left(\mathbf{A}_{0}-\mathbf{K} \mathbf{C}_{0}\right) \tilde{\boldsymbol{\eta}}_{0}+\mathbf{B}_{0} \mathbf{J}\left(\psi_{y}\right) \tilde{\mathbf{v}} \\
\dot{\tilde{\mathbf{b}}} & =-\mathbf{T}^{-1} \tilde{\mathbf{b}}-\frac{1}{\gamma} \mathbf{K}_{3} \tilde{\mathbf{y}} \\
\mathbf{M} \dot{\tilde{\mathbf{v}}} & =-\mathbf{D} \tilde{\boldsymbol{v}}+\mathbf{J}^{T}\left(\psi_{y}\right) \tilde{\mathbf{b}}-\frac{1}{\gamma} \mathbf{J}^{T}\left(\psi_{y}\right) \mathbf{K}_{4} \tilde{\mathbf{y}}
\end{aligned}
$$

The dynamics of the velocity estimation error (57) can be rewritten as:

$$
\mathbf{M} \dot{\tilde{v}}=-\mathbf{D} \tilde{\boldsymbol{v}}-\frac{1}{\gamma} \mathbf{J}^{T}\left(\psi_{y}\right) \tilde{\mathbf{z}}
$$

where

$$
\tilde{\mathbf{z}} \triangleq \mathbf{K}_{4} \tilde{\mathbf{y}}-\gamma \tilde{\mathbf{b}}
$$

By defining

$$
\tilde{\mathbf{x}} \triangleq\left[\begin{array}{c}
\tilde{\boldsymbol{\eta}}_{0} \\
\tilde{\mathbf{b}}
\end{array}\right]
$$




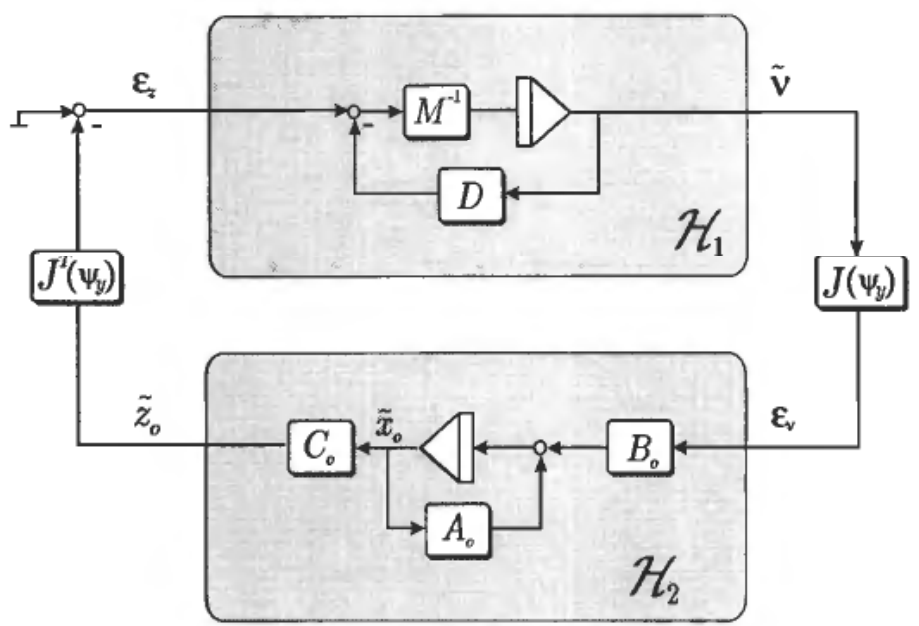

Figure 8. Block diagram showing the dynamics of the position/bias and velocity estimation errors.

(55), (56) and (58) can be written in compact form as:

$$
\begin{aligned}
\dot{\tilde{\mathbf{x}}} & =\mathbf{A} \tilde{\mathbf{x}}+\mathbf{B J}\left(\psi_{y}\right) \tilde{\boldsymbol{v}} \\
\tilde{\mathbf{z}} & =\mathbf{C} \tilde{\mathbf{x}}
\end{aligned}
$$

where

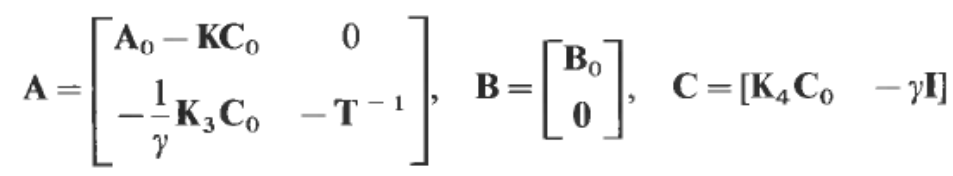

The error dynamics is shown in Figure 8 where two new error terms $\varepsilon_{z}=-\mathbf{J}^{T}(\mathbf{y}) \tilde{\mathbf{z}}$ and $\boldsymbol{\varepsilon}_{v}=\mathbf{J}(\mathbf{y}) \tilde{v}$ are defined.

\subsubsection{SPR-Lyapunov Analysis}

The observer gains are determined by using an SPR-Lyapunov design.

Lemma 3.1 (Kalman-Yakubovich-Popov (KYP) Lemma) Let $Z(s)=\mathscr{C}(s I-\mathscr{A})^{-1} \mathscr{B}$ be a $n \times n$ transfer function matrix, where $\mathscr{A}$ is Hurwitz, $(\mathscr{A}, \mathscr{B})$ is controllable, and $(\mathscr{A}, \mathscr{C})$ is observable. Then, $Z(s)$ is strictly positive real (SPR) if and only if there exist positive definite matrices $\mathscr{P}=\mathscr{P}^{T}$ and $\mathscr{Q}=\mathscr{Q}^{T}$ such that (Khalil (1996)):

$$
\begin{aligned}
\mathscr{P} \mathscr{A}+\mathscr{A}^{T} \mathscr{P} & =-\mathscr{Q} \\
\mathscr{B}^{T} \mathscr{P} & =\mathscr{C}
\end{aligned}
$$

Proposition 3.1 (SPR block $\mathcal{H}_{2}$ ) If $\gamma>0$ and the observer gain matrices are given the following structure: 


$$
\begin{aligned}
\mathbf{K}_{1} & =\left[\begin{array}{ccc}
k_{11} & 0 & 0 \\
0 & k_{12} & 0 \\
0 & 0 & k_{13} \\
k_{14} & 0 & 0 \\
0 & k_{15} & 0 \\
0 & 0 & k_{16}
\end{array}\right], \mathbf{K}_{3}=\left[\begin{array}{ccc}
k_{31} & 0 & 0 \\
0 & k_{32} & 0 \\
0 & 0 & k_{33}
\end{array}\right] \\
\mathbf{K}_{2} & =\left[\begin{array}{ccc}
k_{21} & 0 & 0 \\
0 & k_{22} & 0 \\
0 & 0 & k_{23}
\end{array}\right], \mathbf{K}_{4}=\left[\begin{array}{ccc}
k_{41} & 0 & 0 \\
0 & k_{42} & 0 \\
0 & 0 & k_{43}
\end{array}\right]
\end{aligned}
$$

then the elements $k_{i j}>0$ can be chosen such that the triple (A, B, C) given by (61), that is the mapping $\varepsilon_{\mathbf{v}} \mapsto \tilde{\mathbf{z}}$ (Block $\mathcal{H}_{1}$ in Figure 8 ) satisfies the KYP lemma.

Proof. Since $\mathbf{K}_{3}$ and $\mathbf{K}_{4}$ are chosen to be diagonal, the mapping $\boldsymbol{\varepsilon}_{\mathbf{v}} \mapsto \tilde{\mathbf{z}}$ (see the lower block in Figure 8) can be described by three decoupled transfer functions:

and:

$$
\tilde{\mathbf{z}}(s)=\mathbf{H}(s) \varepsilon_{v}(s) \text { where } \mathbf{H}(s)=\mathbf{H}_{0}(s) \mathbf{H}_{B}(s)
$$

$$
\begin{aligned}
& \mathbf{H}_{0}(s)=\mathbf{C}_{0}\left(s \mathbf{I}+\mathbf{A}_{0}-\mathbf{K C}_{0}\right)^{-1} \mathbf{B}_{0} \\
& \mathbf{H}_{B}(s)=\mathbf{K}_{4}+\left(s \mathbf{I}+\mathbf{T}^{-1}\right)^{-1} \mathbf{K}_{\mathbf{3}}
\end{aligned}
$$

The transfer functions $h_{0}^{i}(s)(i=1 \ldots 3)$ of $\mathbf{H}_{0}(s)$ and $h_{B}^{i}(s)(i=1 \ldots 3)$ of $\mathbf{H}_{B}(s)$ becomes:

$$
\begin{gathered}
h_{0}^{i}(s)=\frac{s^{2}+2 \zeta_{i} \omega_{o i} s+\omega_{o i}^{2}}{s^{3}+\left(k_{1}(i+3)+k_{2 i}+2 \zeta_{i} \omega_{o i}\right) s^{2}+\left(\omega_{o i}^{2}+2 \zeta_{i} \omega_{o i} k_{2 i}-k_{1 i} \omega_{o i}^{2}\right) s+\omega_{o i}^{2} k_{2 i}} \\
s+\left(\frac{1}{T_{i}}+\frac{k_{31}}{k_{4 i}}\right) \\
h_{B}^{i}(s)=k_{4 i} \frac{1}{s+\frac{1}{T_{i}}} T_{i} \gg 1 k_{4 i} \frac{s+\frac{k_{31}}{k_{4 i}}}{s+\frac{1}{T_{i}}}
\end{gathered}
$$

In order to obtain the desired notch effect (wave filtering) of the observer, see Definition 1, the desired shape of $h_{0}^{i}(s)$ is specified as:

$$
h_{0 d}^{i}(s)=\frac{s^{2}+2 \zeta_{i} \omega_{o i} s+\omega_{o i}^{2}}{\left(s^{2}+2 \zeta_{n i} \omega_{o i} s+\omega_{o i}^{2}\right)\left(s+\omega_{c i}\right)}
$$

where $\zeta_{n i}>\zeta_{i}$ determines the notch and $\omega_{c i}>\omega_{o i}$ is the filter cut-off frequency. Equating (66) and (68) yields the following formulas for the filter gains in $\mathbf{K}_{1}$ and $\mathbf{K}_{2}$ :

$$
\begin{aligned}
& k_{1 i}=-2 \omega_{c i}\left(\zeta_{n i}-\zeta_{i}\right) \frac{1}{\omega_{o i}} \\
& k_{2 i}=2 \omega_{o i}\left(\zeta_{n i}-\zeta_{i}\right) \\
& k_{3 i}=\omega_{c i}
\end{aligned}
$$

Notice that the filter gains can be gain-scheduled with respect to the dominating wave frequencies $\omega_{o i}$ if desired. In Figure 9 the total transfer function $h^{i}(s)=h_{\mathrm{B}}^{i}(s) \cdot h_{\mathrm{O}}^{i}(s)$ is 

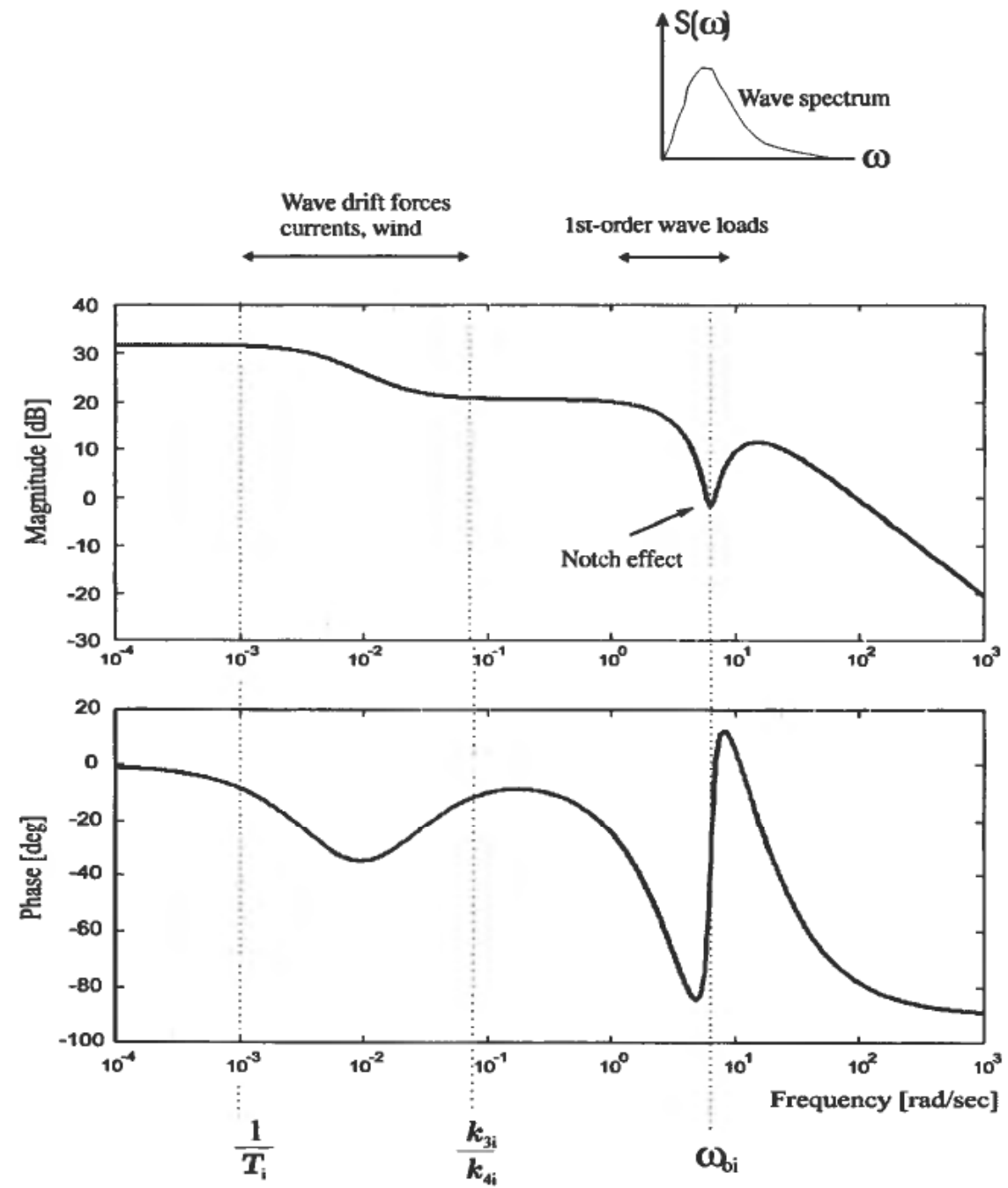

Figure 9. Bode plot showing the transfer function $h^{i}(s)$ when $1 / T_{i} \ll \lambda_{i} / \kappa_{i}<\omega_{o i}<\omega_{c i}(i=1 \ldots 3)$.

illustrated when all filter gains are properly selected. It is important that the 3 decoupled transfer functions $h^{i}(s)$ all have phase greater than $-90^{\circ}$ in order to meet the $S P R$ requirement. It turns out that the KYP lemma and therefore the $S P R$ requirement can easily be satisfied if the following tuning rules for $T_{i}, \lambda_{i}$ and $\kappa_{i}$ are applied:

$$
1 / T_{i} \ll k_{3 i} / k_{4 i}<\omega_{o i}<\omega_{c i}, \quad(i=1 \ldots 3) .
$$

Here $\omega_{o i}(i=1 \ldots 3)$ are the dominating wave frequencies and $T_{i} \gg 1(i=1 \ldots 3)$ are the bias time constants used to specify the limited integral effect in the bias estimator.

Remark 3.2 The observer can be gain-scheduled with respect to the dominating wave frequency vector $\omega_{o}=\left[\omega_{o 1}, \omega_{o 2}, \omega_{o 3}\right]^{T}$ by noticing that $\mathbf{K}_{1}=\mathbf{K}_{1}\left(\omega_{o}\right)$, see Eqs. (69)-(70). The gain matrices $\mathbf{K}_{2}, \mathbf{K}_{3}$ and $\mathbf{K}_{4}$ are independent of $\omega_{o}$. An estimate of $\omega_{o}$ can be found by using an on-line frequency tracker (Fossen (1994)) or parameter adaptation, see Section 3.5. 
Theorem 3.1 (Main Result: GES Nonlinear Observer Error Dynamics) Under Assumptions $A 1-A 4$ the equilibrium point $\left(\tilde{\boldsymbol{\eta}}_{0}, \tilde{\mathbf{b}}, \tilde{\mathbf{v}}\right)=(\mathbf{0}, \mathbf{0}, \mathbf{0})$ of the error dynamics $(55)-(57)$ is GES.

Proof. Consider the following Lyapunov function candidate:

$$
V=\gamma \tilde{\boldsymbol{v}}^{T} \mathbf{M} \tilde{\mathbf{v}}+\tilde{\mathbf{x}}^{T} \mathbf{P} \tilde{\mathbf{x}}
$$

Differentiation of $V$ along the trajectories of $\tilde{\mathbf{v}}$ and $\tilde{\mathbf{x}}$ and application of Assumptions A1-A4, yields:

$$
\dot{V}=-\gamma \tilde{v}^{T}\left(\mathbf{D}+\mathbf{D}^{T}\right) \tilde{v}+\tilde{\mathbf{x}}^{T}\left(\mathbf{P A}+\mathbf{A}^{T} \mathbf{P}\right) \tilde{\mathbf{x}}+2 \tilde{v}^{T} \mathbf{J}^{T}\left(\psi_{y}\right) \mathbf{B}^{T} \mathbf{P} \tilde{\mathbf{x}}-2 \tilde{v}^{T} \mathbf{J}^{T}\left(\psi_{y}\right) \tilde{\mathbf{z}}
$$

Application of Proposition 3.1 to (74), yields:

$$
\dot{V}=-\gamma \tilde{\boldsymbol{v}}^{T}\left(\mathbf{D}+\mathbf{D}^{T}\right) \tilde{\boldsymbol{v}}-\tilde{\mathbf{x}}^{T} \mathbf{Q} \tilde{\mathbf{x}}
$$

Hence, $\tilde{\boldsymbol{v}}$ and $\tilde{\mathbf{x}}=\left[\tilde{\xi}^{T}, \tilde{\boldsymbol{\eta}}^{T}, \tilde{\mathbf{b}}^{T}\right]^{T}$ converge exponentially to zero, q.e.d.

\subsubsection{Passivity Interpretation of the Nonlinear Observer}

The error dynamics in Figure 8 can be described by two blocks $\mathscr{H}_{1}$ and $\mathscr{H}_{2}$ corresponding to the mappings $\varepsilon_{z} \mapsto \tilde{\boldsymbol{v}}$ and $\varepsilon_{\mathbf{v}} \mapsto \tilde{\mathbf{z}}$. Notice that the coordinate transformation is performed through a non-singular and bounded matrix $\mathbf{J}\left(\psi_{y}\right)$.

Proposition 3.2 (Strictly Passive Block $\mathscr{H}_{1}$ ) The mapping $\mathcal{H}_{1}$ is state strictly passive.

Proof. Let

$$
U=\frac{1}{2} \gamma \tilde{v}^{T} \mathbf{M} \tilde{v}
$$

be a positive definite storage function. Time differentiation of $U$ along the trajectories of $\tilde{v}$ yields:

$$
\ddot{U}=-\frac{1}{2} \gamma \tilde{v}^{T}\left(\mathbf{D}+\mathbf{D}^{T}\right) \tilde{v}-\tilde{\mathbf{z}}^{T} \mathbf{J}\left(\psi_{y}\right) \tilde{v}
$$

Using the fact that $\varepsilon_{z}=-\mathbf{J}^{T}\left(\psi_{y}\right) \tilde{\mathbf{z}}$, yields:

$$
\boldsymbol{\varepsilon}_{z}^{T} \tilde{v}=\dot{U}+\frac{1}{2} \gamma \tilde{v}^{T}\left(\mathbf{D}+\mathbf{D}^{T}\right) \hat{v}
$$

Hence:

$$
\int_{t_{0}}^{t} \boldsymbol{\varepsilon}_{z}^{T}(\tau) \tilde{\boldsymbol{v}}(\tau) d \tau \geqslant \alpha \tilde{\boldsymbol{v}}^{T} \tilde{\boldsymbol{v}}+\beta
$$

where $\alpha=(1 / 2) \gamma \lambda_{\min }(\mathbf{M})$ is a positive constant and $\beta=(1 / 2) \gamma \int_{t_{0}}^{t} \tilde{v}^{T}\left(\mathbf{D}+\mathbf{D}^{T}\right) \tilde{v} d \tau \geqslant 0$ is the dissipated energy due to hydrodynamic damping.

Theorem 3.2 (Passive Observer Error Dynamics) The nonlinear observer error dynamics (55)-(57) is passive. 


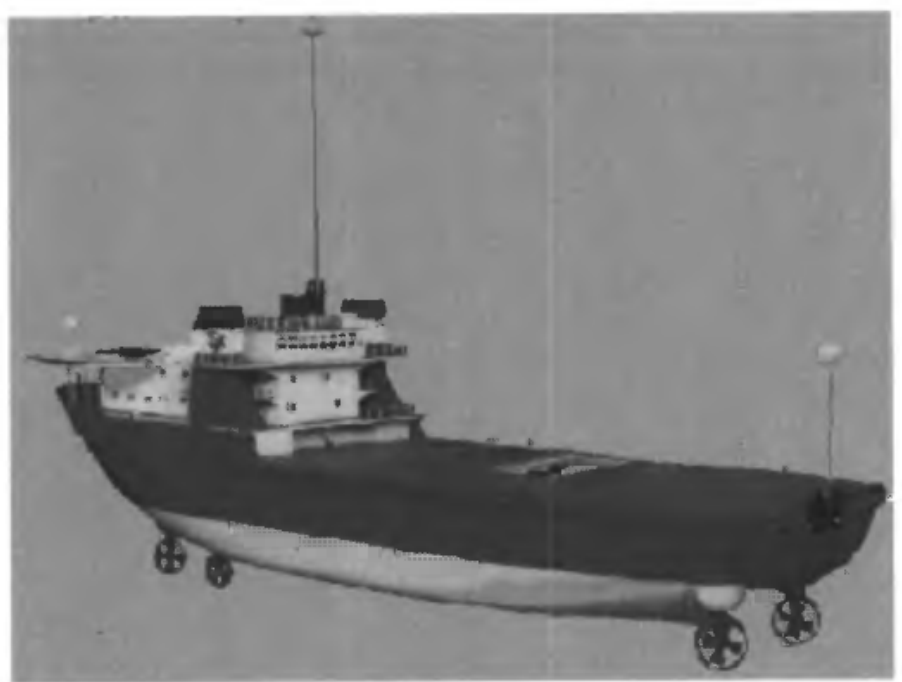

Figure 10. CyberShip I: Model ship scale $1: 70$.

Proof. Since it is established that $\mathscr{H}_{1}$ is state strictly passive and $\mathscr{H}_{2}$ is $S P R$ (Propositions 3.1 and 3.2), the nonlinear observer error dynamics (55) (57) must be passive. In addition to this, GES has been shown (Theorem 3.1).

\subsection{Adaptive Passive Observer Design}

An extension to the case where the wave frequencies $\omega_{i}$ and damping rations $\zeta_{i}$ $(i=1 \ldots 3)$ which are parameters in $\mathbf{A}_{w}$ vary with time-varying sea states are done in Strand and Fossen (1999). This is done by including an adaptive updating mechanism for the wave frequencies. Experimental results with a model ship are also reported. The performance of the adaptive observer is better than the non-adaptive observer mainly due to the improvements of the adaptive wave filter.

Gain-scheduling techniques, using off-line batch processing frequency trackers and external sensors such as wind velocity, wave radars and roll, pitch angle measurements can also be used to adjust the WF model parameters to varying sea states (Fossen (1994)). Additional sensors units can, however, be avoided by using an adaptive observer design. Since the wave models are assumed to be decoupled in surge, sway and yaw, $\Lambda$ and $\Omega$ in $\mathbf{A}_{w}$ are diagonal matrices given by:

$$
\mathbf{A}_{w}\left(\boldsymbol{\theta}_{w}\right)=\left[\begin{array}{cc}
\mathbf{0} & \mathbf{I} \\
-\mathbf{\Omega}^{2} & -2 \Lambda \Omega
\end{array}\right] \triangleq\left[\begin{array}{cc}
\mathbf{0} & \mathbf{I} \\
-\operatorname{diag}\left(\boldsymbol{\theta}_{w 1}\right) & -\operatorname{diag}\left(\boldsymbol{\theta}_{w 2}\right)
\end{array}\right]
$$

where $\theta_{w}=\left[\theta_{w 1}^{T}, \theta_{w 2}^{T}\right]^{T}$, and $\theta_{w 1}, \theta_{w 2} \in \mathbb{R}^{3}$ contain the unknown wave model parameters to be estimated on-line. The adaptive observer equations and the stability analysis are found in Strand and Fossen (1999).

\subsection{Experimental Results}

The nonlinear and adaptive observers have been implemented and tested at the Guidance, Navigation and Control (GNC) Laboratory at the Department of Engineering Cybernetics, NTNU. A detailed description of the laboratory is found in Strand 


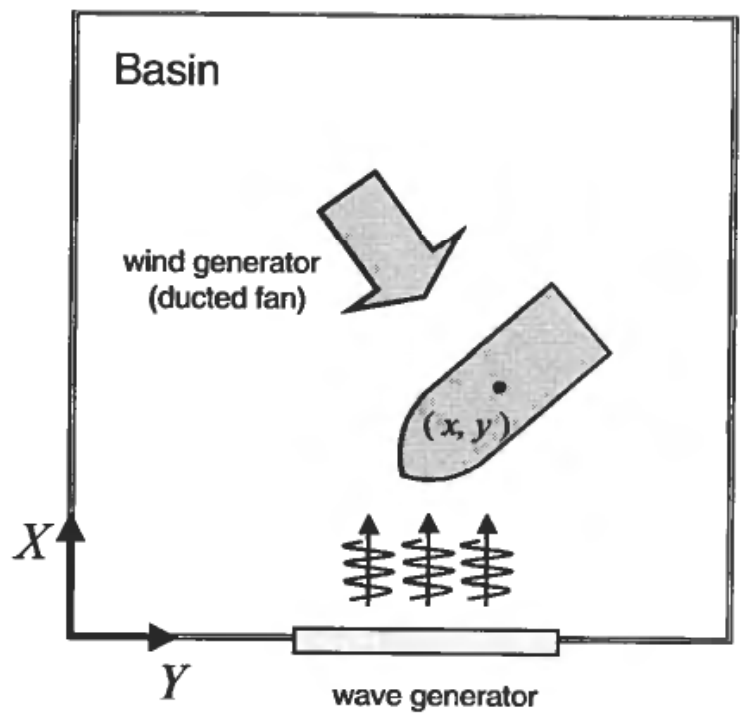

Figure 11. Left: Experimental setup. Right: Picture of Cybership I.

(1999) and URL: http://www.itk.ntnu.nolansatte/Fossen_Thor/GNC/. In the experiments we used Cybership I.

A nonlinear PID controller is used for maintaining the ship at the desired position $\left(x_{d}, y_{d}\right)$ and heading $\psi_{d}$. The PID control law is written:

$$
\tau_{c}=-\mathbf{M J}^{T}\left(\psi_{y}-\psi_{d}\right)\left(\mathbf{K}_{p} \mathbf{e}+\mathbf{K}_{d} \dot{\mathbf{e}}+\mathbf{K}_{i} \int_{0}^{t} \mathbf{e} d \tau\right)
$$

where $\mathbf{e}$ is the estimated LF position deviation, defined as:

$$
\mathbf{e} \triangleq \mathbf{J}^{T}\left(\psi_{d}\right)\left(\hat{\boldsymbol{\eta}}-\boldsymbol{\eta}_{d}\right)
$$

An illustration of the experimental setup is shown in Figure 11. The experimental results are transformed to full scale by requiring that the Froude number:

$$
F_{n}=\frac{U}{\sqrt{L g}}=\text { constant }
$$

Here $U$ is the vessel speed, $L$ is the length of the ship and $g$ is the acceleration of gravity. The scaling factors are given in Table 1 where $m$ is the mass and the subscripts $m$ and $s$ denote the model and the full-scale ship, respectively. The length of the model ship is $L_{m}=1.19$ meters and the mass is $m_{m}=17.6 \mathrm{~kg}$. A full scale ship similar to Cybership I has typically a length of 70-90 meters and mass of 4000-5000 tonnes.

The experiment can be divided into three phases:

- Phase I (No waves). Initially the ship is maintaining the desired position and heading with no environmental loads acting on the ship (calm water). The reference heading is -140 degrees. When the data acquisition starts, a wind fan is switched on. There is no adaptive wave filtering. The effect of the wind loads are reflected in the bias estimates in Figure 13.

- Phase II (Waves, adaptive wave filter is off). After 1700 seconds the wave generator is started. In this phase we can see the performance of the observer without 
Table 1. Scaling factors used in the experiments (Bis scaling).

\begin{tabular}{ll}
\hline position: & $L_{s} / L_{m}$ \\
linear velocity: & $\sqrt{L_{s} / L_{m}}$ \\
angular velocity: & $\sqrt{L_{m} / L_{s}}$ \\
linear acceleration: & 1 \\
angular acceleration: & $L_{m} / L_{s}$ \\
force: & $m_{s} / m_{m}$ \\
moment: & $\frac{m_{s} L_{s}}{m_{m} L_{m}}$ \\
time: & $\sqrt{L_{s} / L_{m}}$ \\
\hline
\end{tabular}
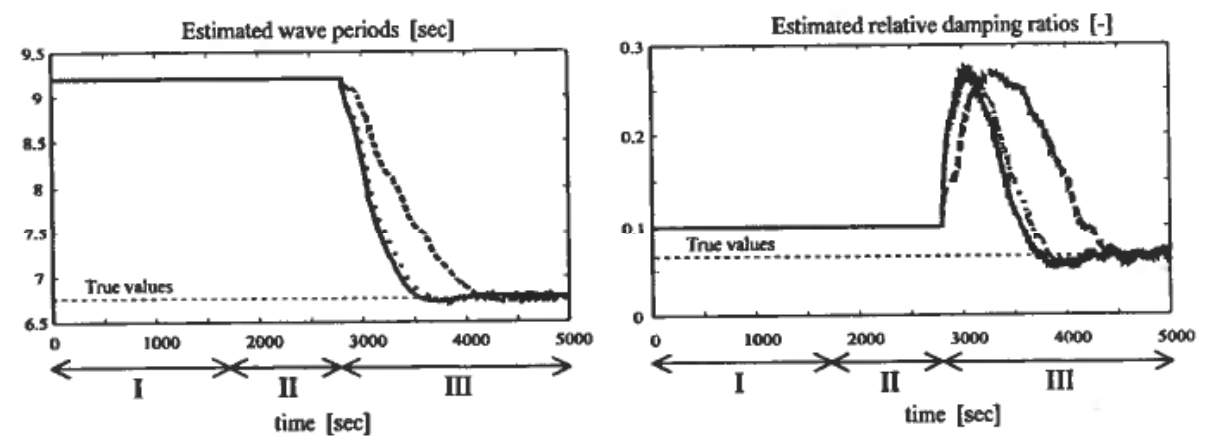

Figure 12. Estimated wave periods (left) and estimated relative damping ratios (right) for surge (solid), sway (dashed) and yaw (dotted). The adaptive wave-filter is activated after 2800 seconds.

adaptive wave filter. In the wave model we are assuming that the dominating wave period is 9.2 seconds and the relative damping ratio is 0.1 , see Figure 12 .

- Phase III (Waves, adaptive wave filter is on). After 2800 seconds the adaptive wave filter is activated. The estimates of dominating wave period and relative damping are plotted in Figure 12 for surge, sway and yaw.

A spectrum analysis of the position and heading measurements shows that the estimated wave periods converge to their true values, that is wave periods of approximately 7.8 seconds and relative damping ratios of 0.07, see Figure 12. In Figure 13 the measured position deviation and heading are plotted together with the corresponding LF estimates. The effect of the adaptive wave filtering is clearly seen in Figure 15, where the innovation signals are significantly reduced during Phase III, when the adaptation is active and the wave model parameters start converging to their true values. The effect of bad wave filtering is reflected by noisy control action by the thrusters during phase II, see Figure 15. A zoom-in of the heading measurement together with the LF estimate is given in Figure 14 both for phase II and III. Here we see that the LF estimates have a significant WF contribution when the adaptive wave filter is off. This is the reason for the noisy control action in phase II. The other zoom- 

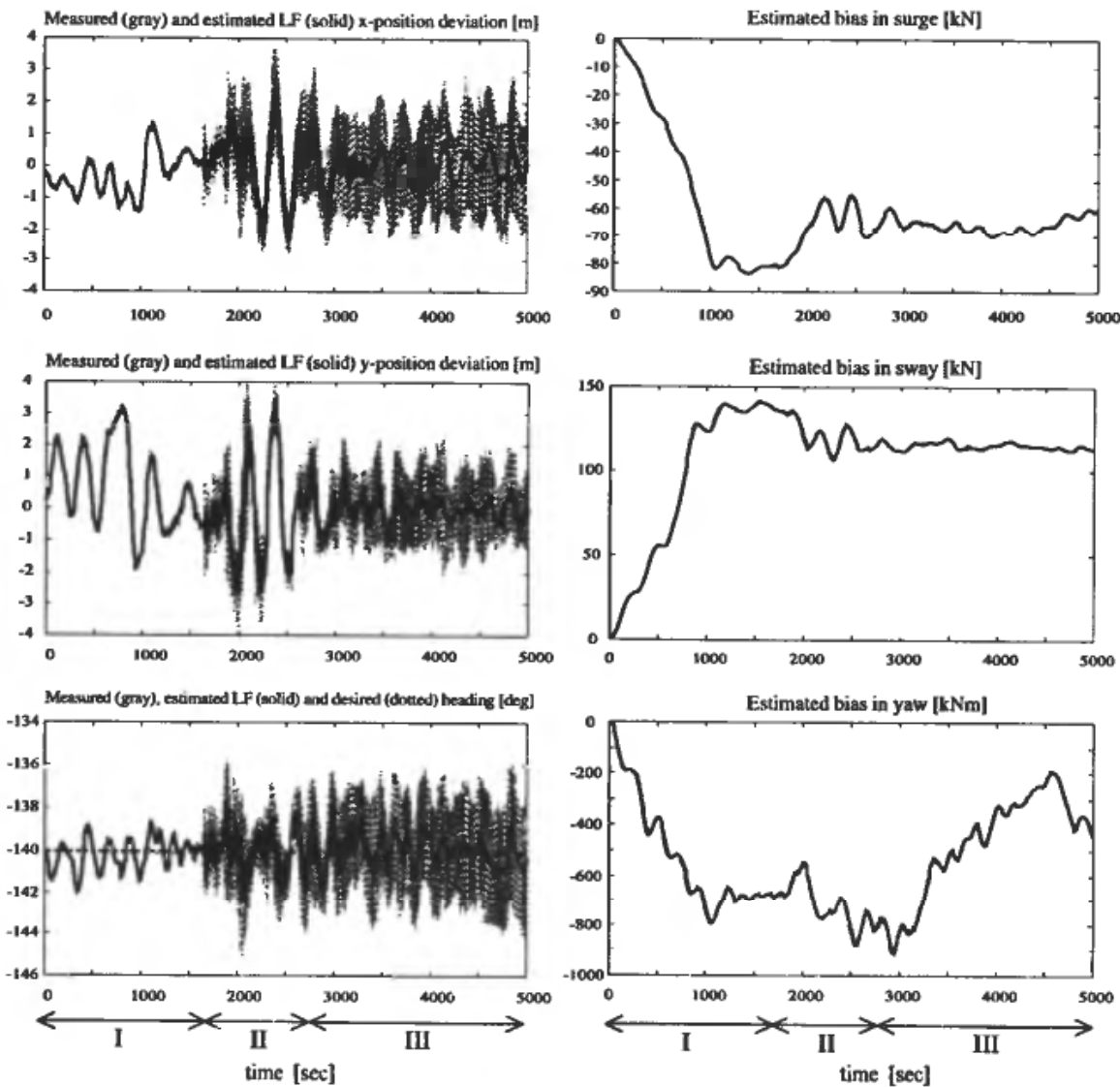

Figure 13. Left column: Measured position and heading (gray) together with corresponding LF estimates (solid). Right column: Estimated bias in surge, sway and yaw.

Zoom-In: Measured (dotted) and estimated LF (solid) heading (dashed) [deg]
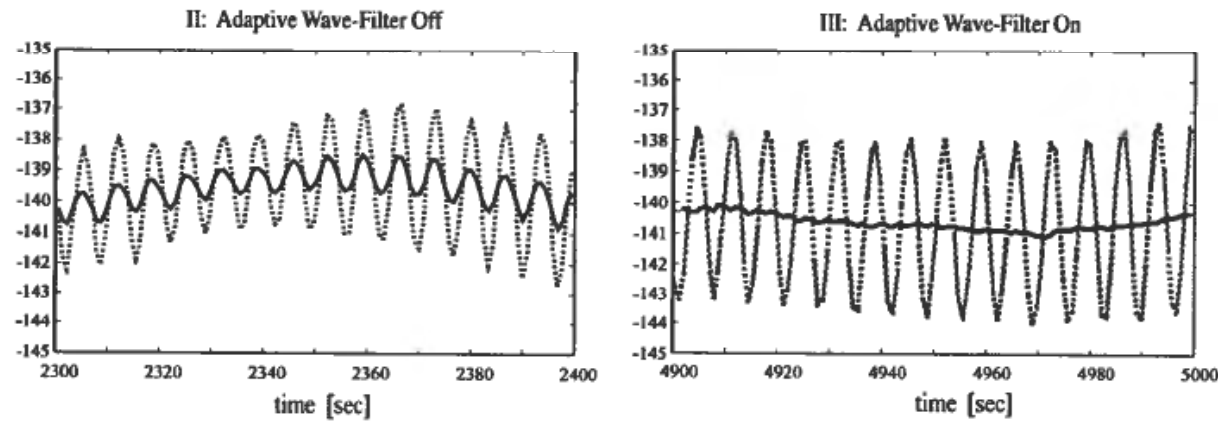

Figure 14. Zoom-in of measured and estimated LF heading. Left: Observer without adaptive wave-filtering. Right: Observer with adaptive wave-filtering. 

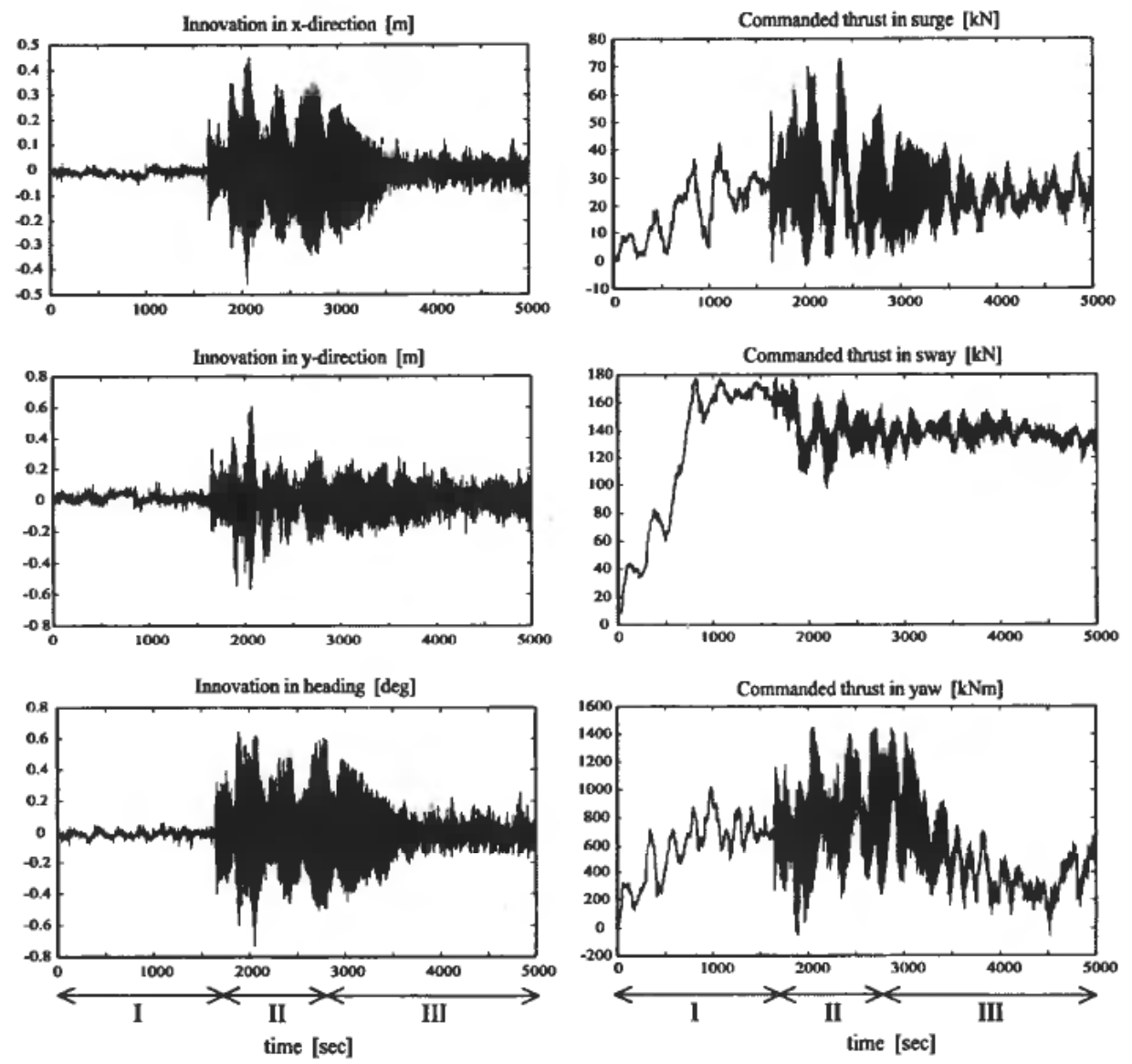

Figure 15. Left column: Innovation in position and heading. Right column: Commanded thrust in surge, sway and yaw.

in shows excellent LF estimation when the adaptive wave filter is active and the wave model parameters have converged to their true values. Hence, it can be concluded that adaptive wave filtering yields a significant improvement in performance compared to filters with fixed WF model parameters operating in varying sea states.

\section{Nonlinear Passive DP}

Dynamic positioning (DP) systems have been commercially available for marine vessels since the 1960s. The first DP systems were designed using conventional PID controllers in cascade with low pass and/or notch filters to suppress the wave-induced motion components. From the middle of the 1970s more advanced control techniques based on optimal control and Kalman-filter theory were proposed (Fossen (1994)).

In the forthcoming sections, we will exploit the nonlinear passive observer of Section 3 and design an output feedback controller for DP. Two design techniques will be discussed:

- Observer backstepping

- Cascaded observer-controller design through a nonlinear separation principle 


\subsection{Observer Backstepping}

Traditional DP and thruster assisted PM systems are usually designed by using model based linear feedback control in combination with linear optimal estimation methods. This traditional design is based on the assumption of linear vessel kinematics and error dynamics. As a result of these assumptions the vessel kinematics must be linearized about several yaw angles, giving different linear models in corresponding heading sectors. To avoid this, attempts towards nonlinear DP and PM control designs have been taken.

In Grøvlen and Fossen (1996) an observer backstepping approach on component form was introduced as an attempt towards a nonlinear DP control law. The design was adapted to a vectorial setting in Fossen and Grøvlen (1998). This design provides noise filtering of measured position and heading together with velocity estimates, under the assumption of white noise only. However, in reality the measurements are corrupted through noise colored by wave induced motions. To avoid wear and tear of the propulsion system the estimates entering the feedback loop should be filtered by using wave filtering techniques. Further, bias state estimation should be present representing unmodeled slowly varying external forces.

This section is based on Aarset, Strand and Fossen (1998) which is an extension of the results of Fossen and Grøvlen (1998) constituting a complete nonlinear control design for DP and PM purposes. Filtering of noise and WF motion, bias state estimates and velocity estimates are done by using the passive observer in Section 3. Integral action is included in the control law by feedback cancellation of bias estimates (separation principle). Additional integral action is included in the controller for performance improvements and compensation of modeling errors. The control design will be based on the model of a moored ship, see Figure 16, and will then apply for both moored and free-floating ships.

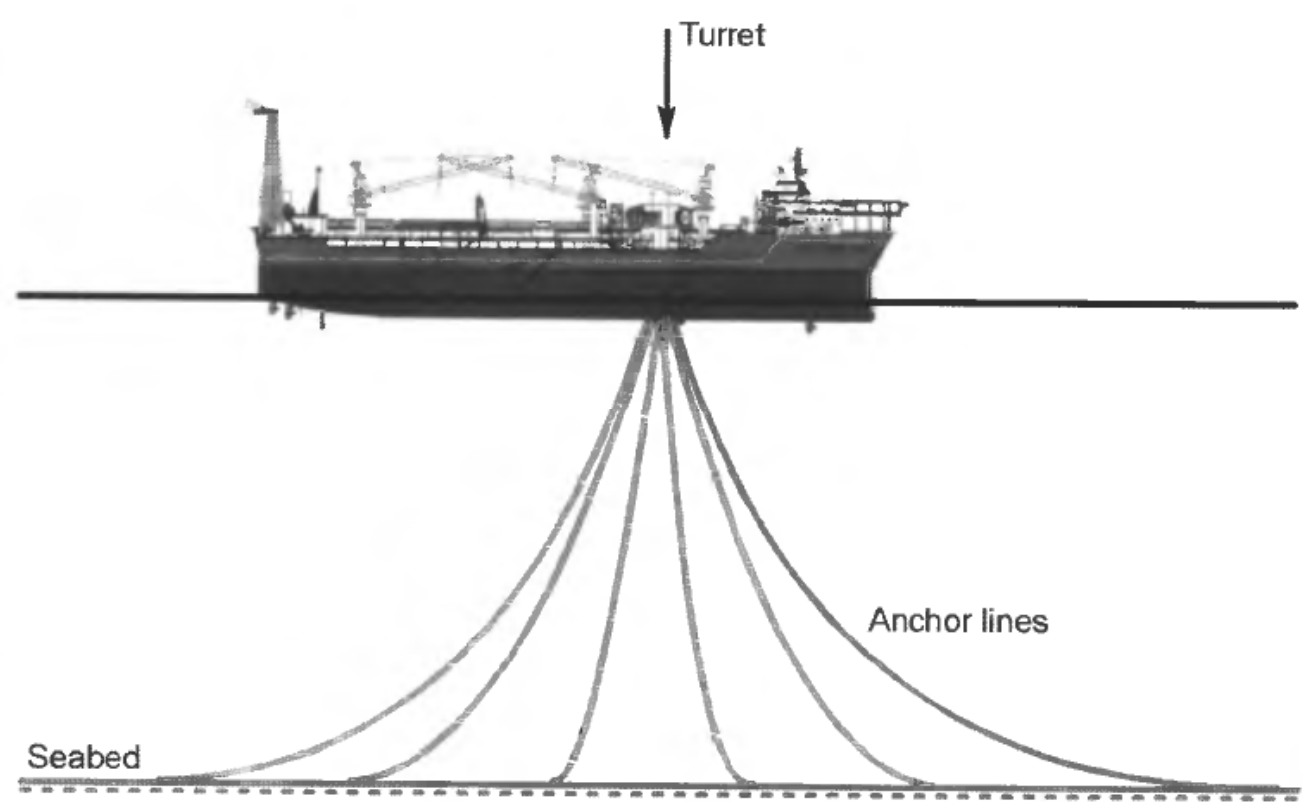

Figure 16. Dynamic positioned ship and mooring system. 


\subsubsection{Main Result}

Consider the nonlinear ship model of Section 2:

$$
\begin{aligned}
\mathbf{M} \dot{v}+\mathbf{D} v+\mathbf{J}^{T}(\psi) \mathbf{G} \boldsymbol{\eta} & =\tau+\mathbf{J}^{T}(\psi) \mathbf{b} \\
\dot{\boldsymbol{\eta}} & =\mathbf{J}(\psi) \boldsymbol{v}
\end{aligned}
$$

where $\mathbf{J}^{T}(\psi) \mathbf{G} \boldsymbol{\eta}$ represents the mooring forces. The passive observer of Section 3 is modified to include the mooring forces according to:

$$
\begin{aligned}
\dot{\hat{\boldsymbol{\xi}}} & =\mathbf{A}_{w} \hat{\boldsymbol{\xi}}+\mathbf{K}_{1} \tilde{\mathbf{y}} \\
\dot{\hat{\boldsymbol{\eta}}} & =\mathbf{J}(\psi) \hat{\boldsymbol{v}}+\mathbf{K}_{2} \tilde{\mathbf{y}} \\
\dot{\hat{\mathbf{b}}} & =-\mathbf{T}-\mathbf{1} \hat{\mathbf{b}}+\mathbf{K}_{\mathbf{3}} \tilde{\mathbf{y}} \\
\mathbf{M} \dot{\hat{\boldsymbol{v}}} & =-\mathbf{D} \hat{\boldsymbol{v}}-\mathbf{J}^{T}(\psi) \mathbf{G} \hat{\boldsymbol{\eta}}+\mathbf{J}^{T}(\psi) \hat{\mathbf{b}}+\tau+\mathbf{J}^{T}(\psi) \mathbf{K}_{4} \tilde{\mathbf{y}} \\
\hat{\mathbf{y}} & =\hat{\boldsymbol{\eta}}+\mathbf{C}_{w} \hat{\boldsymbol{\xi}}
\end{aligned}
$$

This results in the error dynamics:

$$
\begin{aligned}
\dot{\tilde{\xi}} & =\mathbf{A}_{w} \tilde{\xi}-\mathbf{K}_{1} \tilde{\mathbf{y}} \\
\dot{\tilde{\boldsymbol{\eta}}} & =\mathbf{J}(\psi) \tilde{\boldsymbol{v}}-\mathbf{K}_{2} \tilde{\mathbf{y}} \\
\dot{\tilde{\mathbf{b}}} & =-\mathbf{T}^{-1} \tilde{\mathbf{b}}-\mathbf{K}_{3} \tilde{\mathbf{y}} \\
\mathbf{M} \dot{\tilde{v}} & =-\mathbf{D} \tilde{\boldsymbol{v}}-\mathbf{J}^{T}(\psi) \mathbf{G} \tilde{\boldsymbol{\eta}}+\mathbf{J}^{T}(\psi) \tilde{\mathbf{b}}-\mathbf{J}^{T}(\psi) \mathbf{K}_{4} \tilde{\mathbf{y}}
\end{aligned}
$$

which can be compactly written as:

$$
\begin{aligned}
\mathbf{M} \dot{\tilde{\mathbf{v}}} & =-\mathbf{D} \tilde{\mathbf{v}}-\mathbf{J}^{T}(\mathbf{y}) \tilde{\mathbf{z}}_{o} \\
\dot{\tilde{\mathbf{x}}}_{o} & =\mathbf{A}_{o} \tilde{\mathbf{x}}_{o}+\mathbf{B}_{o} \mathbf{J}(\mathbf{y}) \tilde{\boldsymbol{v}} \\
\tilde{\mathbf{z}}_{o} & =\mathbf{C}_{o} \tilde{\mathbf{x}}_{o}
\end{aligned}
$$

where $\tilde{\mathbf{x}}_{o}=\operatorname{col}[\tilde{\xi}, \tilde{\boldsymbol{\eta}}, \tilde{\mathbf{b}}]$ and

$$
\begin{aligned}
& \mathbf{A}_{o}=\left[\begin{array}{ccc}
\mathbf{A}_{w}-\mathbf{K}_{1} \mathbf{C}_{w} & -\mathbf{K}_{1} & \mathbf{0} \\
-\mathbf{K}_{2} \Gamma & -\mathbf{K}_{2} & \mathbf{0} \\
-\mathbf{K}_{3} \Gamma & -\mathbf{K}_{3} & -\mathbf{T}^{-1}
\end{array}\right], \\
& \mathbf{B}_{o}=\left[\begin{array}{l}
\mathbf{0} \\
\mathbf{I} \\
\mathbf{0}
\end{array}\right], \\
& \mathbf{C}_{o}=\left[\begin{array}{lll}
\mathbf{K}_{4} \mathbf{C}_{w} & \mathbf{K}_{4}+\mathbf{G} & -\mathbf{I}
\end{array}\right]
\end{aligned}
$$

Now, observer backstepping can be applied to the observer LF estimates in two vectorial steps. The tracking objective is specified in the Earth-fixed reference frame by defining a smooth trajectory $\eta_{d} \in C^{r}$ which describes the desired position and heading. The backstepping variables are defined as:

$$
\mathbf{z}_{1}=\hat{\boldsymbol{\eta}}-\boldsymbol{\eta}_{d}+\mathbf{K}_{I} \int_{0}^{t}\left(\hat{\boldsymbol{\eta}}-\boldsymbol{\eta}_{d}\right) d \tau \triangleq \dot{\mathbf{e}}_{I}+\mathbf{K}_{I} \mathbf{e}_{I}
$$




$$
\begin{aligned}
\mathbf{z}_{2} & =\mathbf{J}(\psi) \hat{\boldsymbol{v}}-\boldsymbol{\alpha} \\
\dot{\mathbf{e}}_{\boldsymbol{I}} & =\hat{\boldsymbol{\eta}}-\boldsymbol{\eta}_{d} \\
\boldsymbol{\alpha} & =-\mathbf{C}_{1} \mathbf{z}_{1}-\mathbf{D}_{1} \mathbf{z}_{1}+\dot{\boldsymbol{\eta}}_{d}-\mathbf{K}_{\boldsymbol{I}}\left(\hat{\boldsymbol{\eta}}-\boldsymbol{\eta}_{d}\right)
\end{aligned}
$$

where $\mathbf{K}_{I}$ is a positive gain matrix used to obtain integral action. Hence, the control law:

$$
\tau=\mathbf{M} \mathbf{J}^{T}(\psi)\left[-\mathbf{C}_{2} \mathbf{z}_{2}-\mathbf{D}_{2} \mathbf{z}_{2}-\Phi(\cdot)+\mathbf{z}_{1}\right]
$$

where:

$$
\begin{aligned}
\boldsymbol{\Phi}(\cdot)= & \mathbf{J}(\psi)\left[-\mathbf{M}^{-1} \mathbf{J}^{T}(\psi) \mathbf{G} \hat{\eta}-\mathbf{M}^{-1} \mathbf{D} \hat{\boldsymbol{v}}+\mathbf{M}^{-1} \mathbf{J}^{\mathbf{T}}(\psi) \hat{\mathbf{b}}+\mathbf{S}(\hat{\boldsymbol{\omega}}) \hat{v}\right] \\
& +\mathbf{K}_{I} \mathbf{J}(\psi) \hat{\boldsymbol{v}}-\left(\mathbf{C}_{1}+\mathbf{D}_{1}\right)^{2} \mathbf{z}+\left(\mathbf{C}_{1}+\mathbf{D}_{1}\right) \mathbf{z}_{1}-\ddot{\boldsymbol{\eta}}_{d}-\mathbf{K}_{I} \dot{\boldsymbol{\eta}}_{d} \\
\Omega_{1}= & \mathbf{K}_{4}+\mathbf{C}_{1} \mathbf{K}_{2}+\mathbf{K}_{I} \mathbf{K}_{2} \\
= & {\left[\begin{array}{lll}
\omega_{1} & \omega_{2} & \omega_{3}
\end{array}\right]^{T} } \\
\Omega_{2}= & -\mathbf{J}(\psi) \mathbf{S}(\hat{v}) \mathbf{L} \\
= & {\left[\begin{array}{lll}
\omega_{4} & \omega_{5} & \omega_{6}
\end{array}\right]^{T} } \\
\Omega_{2}= & -\mathbf{J}(\psi) \mathbf{S}(\hat{v}) \mathbf{N} \\
= & {\left[\begin{array}{lll}
\omega_{7} & \omega_{8} & \omega_{9}
\end{array}\right]^{T} } \\
\mathbf{L}= & \operatorname{diag}\{0,0,1\} \\
\mathbf{D}_{1}= & \operatorname{diag}\left\{d_{1} \mathbf{k}_{1}^{T} \mathbf{k}_{1}, d_{2} \mathbf{k}_{2}^{T} \mathbf{k}_{2}, d_{3} \mathbf{k}_{3}^{T} \mathbf{k}_{3}\right\}
\end{aligned}
$$

where $\mathbf{k}_{i}(i=1,2,3)$ are the column vectors of the observer gain matrix $\mathbf{K}_{2}$. The diagonal matrix $\mathbf{D}_{2}$ is defined in terms of the elements of $\Omega_{1}, \Omega_{2}, \Omega_{3}$ as:

$$
\begin{aligned}
\mathbf{D}_{2}= & \operatorname{diag}\left\{d_{4}\left(\omega_{1}^{T} \omega_{1}+\omega_{4}^{T} \omega_{4}+\omega_{7}^{T} \omega_{7}\right), d_{5}\left(\omega_{2}^{T} \omega_{2}+\omega_{5}^{T} \omega_{5}+\omega_{8}^{T} \omega_{8}\right),\right. \\
& \left.d_{6}\left(\omega_{3}^{T} \omega_{3}+\omega_{6}^{T} \omega_{6}+\omega_{9}^{T} \omega_{9}\right)\right\}
\end{aligned}
$$

$\mathrm{C}_{2}$ is a positive definite diagonal design matrix. Similarly, $d_{i}(i=4, \ldots, 6)$ are positive design constants. Furthermore, $\mathbf{S}$ is a skew-symmetric matrix defined as:

$$
\mathbf{S}(\mathbf{h})=\left[\begin{array}{ccc}
0 & -h_{3} & h_{2} \\
h_{3} & 0 & -h_{1} \\
-h_{2} & h_{1} & 0
\end{array}\right]
$$

where $\mathbf{h}=\operatorname{col}\left[h_{1}, h_{2}, h_{3}\right]$

\subsubsection{Stability Analysis}

Stability of the controller-observer error dynamics:

$$
\begin{gathered}
\dot{\mathbf{z}}=-\mathbf{C}_{z} \mathbf{z}-\mathbf{D}_{z} \mathbf{z}+\mathbf{E z}+\mathbf{W}_{1} \tilde{\mathbf{y}}+\mathbf{W}_{2} \tilde{\mathbf{v}}+\mathbf{W}_{3} \tilde{\xi} \\
\dot{\mathbf{e}}_{I}=\hat{\boldsymbol{\eta}}-\boldsymbol{\eta}_{d} \\
\mathbf{M} \dot{\tilde{\mathbf{v}}}=-\mathbf{D} \tilde{\boldsymbol{v}}-\mathbf{J}^{T}(\mathbf{y}) \tilde{\mathbf{z}}_{o}
\end{gathered}
$$




$$
\dot{\tilde{\mathbf{x}}}_{o}=\mathbf{A}_{o} \tilde{\mathbf{x}}_{o}+\mathbf{B}_{o} \mathbf{J}(\mathbf{y}) \tilde{\mathbf{v}}
$$

where $\mathbf{z}=\operatorname{col}\left[\mathbf{z}_{1}, \mathbf{z}_{2}\right]$ and:

$$
\begin{gathered}
\mathbf{C}_{z}=\left[\begin{array}{cc}
\mathbf{C}_{1} & \mathbf{0} \\
\mathbf{0} & \mathbf{C}_{2}
\end{array}\right], \quad \mathbf{D}_{z}=\left[\begin{array}{cc}
\mathbf{D}_{1} & \mathbf{0} \\
\mathbf{0} & \mathbf{D}_{2}
\end{array}\right], \quad \mathbf{E}=\left[\begin{array}{cc}
\mathbf{0} & \mathbf{I} \\
-\mathbf{I} & \mathbf{0}
\end{array}\right] \\
\mathbf{W}_{1}=\left[\begin{array}{l}
\mathbf{K}_{2} \\
\Omega_{1}
\end{array}\right], \quad \mathbf{W}_{2}=\left[\begin{array}{c}
\mathbf{0} \\
\Omega_{2}
\end{array}\right], \quad \mathbf{W}_{3}=\left[\begin{array}{c}
\mathbf{0} \\
\Omega_{3}
\end{array}\right]
\end{gathered}
$$

is proven by using Lyapunov theory:

$$
\begin{gathered}
V=V_{\text {con }}+V_{\text {obs }} \\
V_{\text {con }}=\frac{1}{2} \mathbf{z}^{T} \mathbf{z}+\mathbf{e}_{I}^{T} \mathbf{C}_{1} \mathbf{K}_{I} \mathbf{e}_{I} \\
V_{\text {obs }}=\tilde{\boldsymbol{v}}^{T} \mathbf{M} \tilde{v}+\mathbf{x}_{0}^{T} \mathbf{P} \mathbf{x}_{0}
\end{gathered}
$$

Hence, by appropriate choices of the controller-observer gains it can be shown that (Aarset et al. (1998)):

$$
\dot{V} \leqslant 0
$$

If the bias state estimates $\hat{\mathbf{b}}$ are used to cancel out the off-sets in the model (no integral action in the controller $\mathbf{K}_{I}=\mathbf{0}$ ) the output feedback controller will be GES since it can be shown that:

$$
\dot{V}<0
$$

This means that the observer is used to provide integral action through a separation principle. However, in practice it is necessary to include integral action in the controller as well, that is $\mathbf{K}_{I}>0$, in order to obtain good performance. For this case only uniform GS can be guaranteed since $\dot{V} \leqslant 0$. Asymptotic stability can, however, be proven by applying adaptive backstepping where the bias is treated as an unnown constant to be estimated on-line. UGAS is guaranteed through a theorem for non-autonomous systems where $\dot{V}$ only needs to be negative semi-definite, see Loria, Fossen and Teel (1998) or Fossen, Loria and Teel (2000).

Extensions to locally optimal backstepping control and globally inverse optimality are done in Strand, Ezal, Fossen and Kokotovic (1998, 1999).

In the next section, we will show how a more general result for cascaded systems can be used to obtain a nonlinear separation principle for the passive observer and a PD-controller with bias compensation.

\subsection{Cascaded Observer-Controller Design via a Nonlinear Separation Principle}

In this section we will apply a nonlinear separation principle to design an UGAS cascaded observer-controller. Consider a cascaded system in the form (Panteley and Loria (1998)):

$$
\begin{aligned}
& \Sigma_{1}: \dot{\mathbf{x}}_{1}=\mathbf{f}_{1}(t, \mathbf{x})+\mathbf{G}(t, \mathbf{x}) \mathbf{x}_{2} \\
& \Sigma_{2}: \dot{\mathbf{x}}_{2}=\mathbf{f}_{2}(t, \mathbf{x})
\end{aligned}
$$




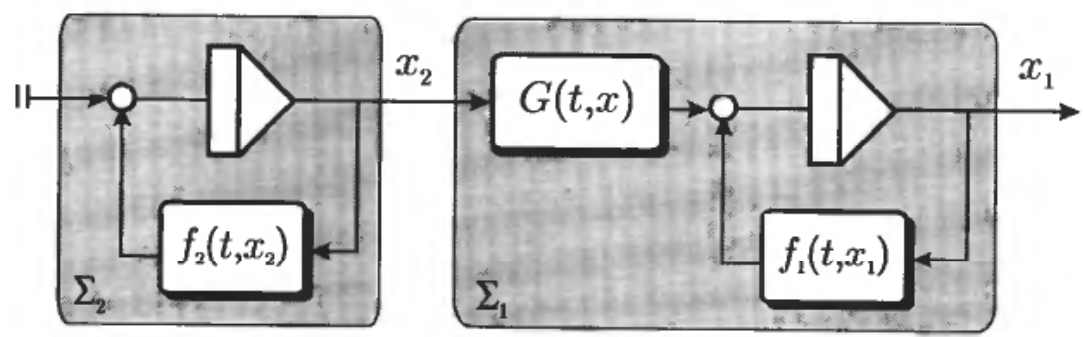

Figure 17. Cascaded systems $\Sigma_{1}$ and $\Sigma_{2}$.

where $\mathbf{x}_{1} \in \mathscr{R}^{n}, \mathbf{x}_{2} \in \mathscr{R}^{n}$ and $\mathbf{x}=\operatorname{col}\left[\mathbf{x}_{1}, \mathbf{x}_{2}\right]$. The function $\mathbf{f}_{1}(t, \mathbf{x})$ is assumed to be continuously differentiable in $\left(t, \mathbf{x}_{1}\right)$ and $\mathbf{f}_{2}(t, \mathbf{x}), \mathbf{G}(t, \mathbf{x})$ are continuous in their arguments, and locally Lipschitz. The cascaded system is shown in Figure 17.

Theorem 4.1 (UGAS Cascaded System) The cascaded systems $\Sigma_{1}$ and $\Sigma_{2}$ given by (123) (124) are UGAS if:

(AI) The system $\Sigma_{2}$ is $U G A S$ and for all $t_{0} \geqslant 0$,

$$
\int_{0}^{\infty}\left\|\mathbf{x}_{2}\left(t, t_{0}, \mathbf{x}_{2}\left(t_{0}\right)\right)\right\| d t \leqslant \phi\left(\left\|\mathbf{x}_{2}\left(t_{0}\right)\right\|\right)
$$

where $\phi(\cdot)$ is a class $\mathscr{K}$ function.

(A2) The system $\dot{\mathbf{x}}_{1}=\mathbf{f}_{1}(t, \mathbf{x})$ is UGAS with a Lyapunov function $V\left(t, \mathbf{x}_{1}\right)$, $V: \mathscr{R} \mathscr{R}_{+} \times \mathscr{R}^{n} \rightarrow \mathscr{R}_{+}$, positive definite (that is $\mathrm{V}(\mathrm{t}, \mathbf{0})=0$ and $\mathrm{V}\left(\mathrm{t} . \mathrm{x}_{1}\right)>0$ for all $\mathbf{x}_{1} \neq \mathbf{0}$ ) and radially unbounded. In addition $V\left(t, x_{1}\right)$ must satisfy:

$$
\left\|\frac{\partial V}{\partial \mathbf{x}_{1}}\right\|\left\|\mathbf{x}_{1}\right\| \leqslant c_{1} V\left(t, \mathbf{x}_{1}\right), \quad \forall\left\|\mathbf{x}_{1}\right\| \geqslant \eta
$$

where $c_{1}, \eta>0$. It is also assumed that $\partial V / \partial \mathbf{x}_{1}\left(t, \mathbf{x}_{1}\right)$ is bounded uniformly in $t$ for all $\left\|\mathbf{x}_{1}\right\| \leqslant \eta$, that is, there exists a constant $c_{2}>0$ such that for all $t \geqslant t_{0} \geqslant 0$ :

$$
\left\|\frac{\partial V}{\partial \mathbf{x}_{1}}\right\| \leqslant c_{2}, \quad \forall\left\|\mathbf{x}_{1}\right\| \leqslant \eta
$$

(A3) The function $\mathbf{g}(t, \mathbf{x})$ satisfies

$$
\|\mathbf{G}(t, \mathbf{x})\| \leqslant \theta_{1}\left(\left\|\mathbf{x}_{2}\right\|\right)+\theta_{2}\left(\left\|\mathbf{x}_{2}\right\|\right)\left\|\mathbf{x}_{1}\right\|
$$

where $\theta_{1}, \theta_{2}: \mathscr{R}_{+} \rightarrow \mathscr{R}_{+}$are continuous.

Proof. See Theorem 2 in Panteley and Loria (1998).

Thanks to this theorem an observer-controller for station-keeping of ships and rigs can be designed in three steps by considering the error dynamics as a cascaded system (Loria, Fossen and Panteley (2000)):

1. Design of a UGAS observer represented by the error dynamics: $\dot{\mathbf{x}}_{2}=. \mathbf{f}_{2}(t, \mathbf{x})$ and check Assumption A1. 
2. Design a UGAS full state feedback controller represented by the error dynamics: $\dot{\mathbf{x}}_{1}=\mathbf{f}_{1}(t, \mathbf{x})$ and check Assumption A2.

3. Replace the states $x$ in the controllers with the estimated states $\hat{x}$ and write the error dynamics of the closed loop system in the form $\dot{\mathbf{x}}_{1}=\mathbf{f}_{1}(t, \mathbf{x})+\mathbf{G}(t, \mathbf{x}) \mathbf{x}_{2}$ where $\mathbf{G}(t, \mathbf{x}) \mathbf{x}_{2}$ represents error terms due to the estimation errors. Check the growth rate condition given by Assumption A3.

\subsubsection{Main Result}

The output feedback controller (PD-type + bias compensation):

$$
\begin{aligned}
& \hat{\mathbf{e}}=\hat{\boldsymbol{\eta}}-\boldsymbol{\eta}_{d} \\
& \tau=-\mathbf{J}^{T}(\psi(t)) \mathbf{K}_{P} \hat{\mathbf{e}}-\mathbf{K}_{d} \hat{\boldsymbol{v}}-\mathbf{J}^{T}(\psi(t)) \hat{\mathbf{b}}
\end{aligned}
$$

where $\boldsymbol{\eta}_{d}=$ constant is UGAS when used in conjecture with the passive nonlinear observer in Section 3 (Loria, Fossen and Panteley (2000)).

\subsubsection{Stability Analysis}

We will demonstrate the 3 steps design procedure by reviewing the results of Loria, Fossen and Panteley (2000). In this work the passive observer of Section 3 represents the $\mathbf{x}_{2}$-dynamics. The observer error dynamics is conveniently written as:

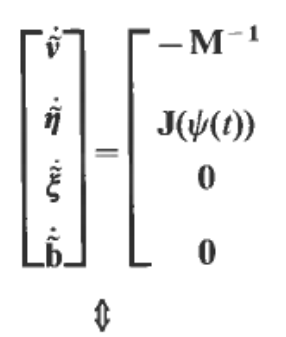

$$
\left.\begin{array}{ccc}
-\frac{1}{\gamma} \mathbf{M}^{-1} \mathbf{J}(\psi(t)) \mathbf{K}_{4} & -\frac{1}{\gamma} \mathbf{J}^{T}(\psi(t)) \mathbf{K}_{3} \mathbf{J}(\psi(t)) & \mathbf{M}^{-1} \mathbf{J}^{T}(\psi(t)) \\
-\mathbf{K}_{2} & -\mathbf{K}_{2} \mathbf{C}_{w} & \mathbf{0} \\
-\mathbf{K}_{1} & \mathbf{A}_{w}-\mathbf{K}_{1} \mathbf{C}_{w} & \mathbf{0} \\
-\frac{1}{\gamma} \mathbf{K}_{4} & 0 & -\mathbf{T}^{-1}
\end{array}\right]\left[\begin{array}{c}
\tilde{\mathbf{v}} \\
\tilde{\boldsymbol{\eta}} \\
\tilde{\xi} \\
\tilde{\mathbf{b}}
\end{array}\right]
$$$$
\dot{\mathbf{x}}_{2}=\mathbf{A}_{o}(\psi(t)) \mathbf{x}_{2}
$$

where $|\psi(t)| \leqslant \pm \pi$ is bounded. The observer error dynamics is UGES and therefore UGAS if the observer gains $\mathbf{K}_{i}(i=1,2,3,4)$ are chosen such that:

$$
\mathbf{Q}_{o}(\psi(t))=-\left(\mathbf{A}_{o}(\psi(t))+\mathbf{A}_{o}^{T}(\psi(t))>0, \quad \forall t\right.
$$

Moreover $V_{o}=\mathbf{x}_{2}^{T} \mathbf{x}_{2}$ yields $\dot{V}_{o}=-\mathbf{x}_{2}^{T} \mathbf{Q}_{o}(\psi(t)) \mathbf{x}_{2}<0, \forall \mathbf{x}_{2} \neq \mathbf{0}$. UGES can also be obtained by using the passivation design in Section 3. Hence, it is straightforward to verify that Assumption $\mathrm{A} 1$ is satisfied since $\left\|\mathbf{x}_{2}(t)\right\| \leqslant \lambda_{1}\left\|\mathbf{x}_{2}\left(t_{0}\right)\right\| e^{-\lambda_{2}\left(c^{t-t_{0}}\right)}$ where $\lambda_{1}$ and $\lambda_{2}$ are two nonnegative constants. Therefore we can choose $\phi\left(\left\|\mathbf{x}_{2}\left(t_{0}\right)\right\|\right)=$ $\lambda_{1} / \lambda_{2}\left\|\mathbf{x}_{2}\left(t_{0}\right)\right\|$.

Next, consider the full-state feedback controller:

$$
\begin{aligned}
\mathbf{e} & =\boldsymbol{\eta}-\boldsymbol{\eta}_{d} \\
\tau^{*} & =-\mathbf{J}^{T}(\psi(t)) \mathbf{K}_{p} \mathbf{e}-\mathbf{K}_{d} \boldsymbol{v}-\mathbf{J}^{T}(\psi(t)) \mathbf{b}
\end{aligned}
$$

where $\dot{\boldsymbol{\eta}}_{d}=$ constant. Hence: 


$$
\begin{aligned}
V_{c} & =\frac{1}{2}\left(v^{T} \mathbf{M} v+\mathbf{b}^{T} \mathbf{b}+\xi^{T} \xi+\mathbf{e}^{T} \mathbf{K}_{p} \mathbf{e}\right) \\
\dot{V}_{c} & =v^{T} \mathbf{M} \dot{v}+\mathbf{b}^{T} \dot{\mathbf{b}}+\xi^{T} \dot{\xi}+\mathbf{e}^{T} \mathbf{K}_{p} \dot{\mathbf{e}} \\
& =-v^{T}\left(\mathbf{D}+\mathbf{K}_{d}\right) v-\frac{1}{2} \mathbf{b}^{T}\left(\mathbf{T}^{-1}+\mathbf{T}^{-T}\right) \mathbf{b}+\frac{1}{2} \xi^{T}\left(\mathbf{A}_{w}+\mathbf{A}_{w}^{T}\right) \xi \\
& \leqslant 0
\end{aligned}
$$

GAS of the origin $\mathbf{e}=\mathbf{0}$ then follows by invoking Krasovskii-LaSalle's invariance principle. Notice that $\dot{V}_{c} \equiv 0$ is equivalent to $v=\xi=\mathbf{b}=\mathbf{0}$. The closed-loop error dynamics is:

$$
\begin{aligned}
& {\left[\begin{array}{c}
\dot{\boldsymbol{v}} \\
\dot{\mathbf{e}} \\
\dot{\boldsymbol{\xi}} \\
\dot{\mathbf{b}}
\end{array}\right]=\left[\begin{array}{cccc}
-\mathbf{M}^{-1}\left(\mathbf{D}+\mathbf{K}_{d}\right) & -\mathbf{M}^{-1} \mathbf{J}^{T}(\psi(t)) \mathbf{K}_{p} & 0 & \mathbf{0} \\
\mathbf{J}(\psi(t)) & \mathbf{0} & \mathbf{0} & \mathbf{0} \\
\mathbf{0} & \mathbf{0} & \mathbf{A}_{w}+\mathbf{A}_{w}^{T} & \mathbf{0} \\
\mathbf{0} & 0 & \mathbf{0} & -\left(\mathbf{T}^{-1}+\mathbf{T}^{-T}\right)
\end{array}\right]\left[\begin{array}{c}
v \\
\mathbf{e} \\
\xi \\
\mathbf{b}
\end{array}\right]} \\
& \hat{\imath} \\
& \dot{\mathbf{x}}_{1}=\mathbf{A}_{c}\left(\mathbf{x}_{1}\right) \mathbf{x}_{1}
\end{aligned}
$$

The conditions of Assumption A2 are easily verified by noticing that $V_{c}=\mathbf{x}_{1}^{T} \mathbf{P}_{c} \mathbf{x}_{1}$ with $\mathbf{P}_{c}=$ block-diag $\left\{\mathbf{M}, \mathbf{K}_{p}, \mathbf{I}, \mathbf{I}\right\}$, yields $\dot{V}_{c}=-\mathbf{x}_{1}^{T} \mathbf{Q}_{c} \mathbf{x}_{1}$ with $\mathbf{Q}_{c}=$ block-diag $\left\{\mathbf{D}+\mathbf{K}_{d}, \mathbf{0},-\left(\mathbf{A}_{w}+\mathbf{A}_{w}^{T}\right), \mathbf{T}^{-1}+\mathbf{T}^{-T}\right\}$. Hence,

$$
c_{1} \geqslant \frac{\max \left\{m_{M}, k_{p M}, 1\right\}}{\min \left\{m_{m}, k_{p m}, 1\right\}}
$$

where $m_{M}$ and $k_{p M}$ are the maximum eigenvalues of $\mathbf{M}$ and $\mathbf{K}_{p}$, and $m_{m}$ and $k_{p m}$ are the minimum eigenvalues of $\mathbf{M}$ and $\mathbf{K}_{p}$. We also see that

$$
c_{2}=\max \left\{m_{M}, k_{p M}, 1\right\}
$$

Finally, we replace the states variables in the controller $\tau^{*}$ with the estimated states. Moreover:

$$
\begin{aligned}
& \hat{\mathbf{e}}=\hat{\boldsymbol{\eta}}-\boldsymbol{\eta}_{d} \\
& \boldsymbol{\tau}=-\mathbf{J}^{T}(\psi(t)) \mathbf{K}_{P} \hat{\mathbf{e}}-\mathbf{K}_{d} \hat{\mathbf{v}}-\mathbf{J}^{T}(\psi(t)) \hat{\mathbf{b}}
\end{aligned}
$$

implying that:

$$
\tau=\tau^{*}+\mathbf{G}\left(\mathbf{x}_{1}\right) \mathbf{x}_{2}
$$

This gives us the expression for:

$$
\mathbf{G}(\mathbf{x})=\left[\mathbf{K}_{d}, \mathbf{J}^{T}(\psi(t)) \mathbf{K}_{p}, \mathbf{0}, \mathbf{J}^{T}(\psi(t))\right]
$$

The growth rate condition of Assumption $\mathrm{A} 3$ is satisfied with $\theta_{1}=$ constant and $\theta_{2}=0$ since the rotation matrix $\mathbf{J}(\psi(t))$ is bounded for all $|\psi(t)| \leqslant \pi$.

\subsubsection{Bias Compensation versus Integral Action}

Notice that the control law (142) compensates the unknown bias $\mathbf{b}=$ constant by using the observer estimate $\hat{\mathbf{b}}$ (integral of the estimation error) and a nonlinear 
separation principle. An alternative approach is to use a cascaded design and constant parameter adaptation to estimate b. Consider the integral controller motivated by (142):

$$
\begin{aligned}
\tau & =-\mathbf{J}^{T}(\psi(t)) \mathbf{K}_{P} \hat{\mathbf{e}}-\mathbf{K}_{d} \hat{\boldsymbol{v}}-\mathbf{J}^{T}(\psi(t)) \hat{\mathbf{b}}^{*} \\
\dot{\mathbf{b}}^{*} & =\Gamma \mathbf{J}(\psi(t)) v, \quad \Gamma>0
\end{aligned}
$$

where integral action is obtained by using the estimate $\hat{\mathbf{b}}^{*}$ (integral of the state variables/ tracking errors). In this case the closed-loop system $\dot{\mathbf{x}}_{1}=\mathbf{f}_{\mathbf{1}}(t, \mathbf{x})$ takes the form:

$$
\begin{aligned}
\mathbf{M} \dot{v}+\left(\mathbf{D}+\mathbf{K}_{d}\right) \boldsymbol{v}+\mathbf{J}^{T}(\psi(t)) \mathbf{K}_{p} \mathbf{e} & =-\mathbf{J}^{T}(\psi(t)) \tilde{\mathbf{b}}^{*} \\
\dot{\tilde{\mathbf{b}}}^{*} & =\Gamma \mathbf{J}(\psi(t)) \boldsymbol{v}
\end{aligned}
$$

where $\tilde{\mathbf{b}}^{*}=\hat{\mathbf{b}}^{*}-\mathbf{b}^{*}$. A Lyapunov function $V_{c}=W_{c}+1 / 2\left(\tilde{\mathbf{b}}^{*}\right)^{T} \Gamma^{-1} \tilde{\mathbf{b}}^{*}$ where $W_{c}=\mathbf{x}_{1}^{T} \mathbf{P}_{c} \mathbf{x}_{1}$ and $\mathbf{P}_{c}=$ block-diag $\left\{\mathbf{M}, \mathbf{K}_{p}, \mathbf{I}, \mathbf{I}\right\}$, yields $\dot{V}_{c}=-\mathbf{x}_{1}^{T} \mathbf{Q}_{c} \mathbf{x}_{1} \leqslant 0$ with $\mathbf{Q}_{c}=$ block-diag $\left\{\mathbf{D}+\mathbf{K}_{d}, \mathbf{0},-\left(\mathbf{A}_{\mathbf{w}}+\mathbf{A}_{w}^{T}\right), \mathbf{T}^{-1}+\mathbf{T}^{-\mathbf{T}}\right\}$. Again UGAS can be proven and convergence of by $\hat{\mathbf{b}}^{*}$ to $\mathbf{b}$ by using the result of Loria, Fossen and Teel (1999) or Fossen, Loria and Teel (2000) for nonlinear nonautonomous systems where $V>0$ and $\dot{V} \leqslant 0$ is only negative semi-definite.

In a practical design it is advantageous to use $\hat{\mathbf{b}}^{*}$ instead of $\hat{\mathbf{b}}$ since the integral of the observer estimation error is more high frequent and therefore oscillate more in steady-state than the signal $\hat{\mathbf{b}}^{*}$. This is observed in regulation of full scale ships (DP) where the desired position and heading reference are kept constant. The same type of oscillation is also documented in experiments with a model ship, see Loria, Fossen and Panteley (1999) for more details. The main reason for this is the stochastic behavior of the environmental disturbances (wind, waves and currents). These phenomena are hardly observed in a deterministic computer simulation.

\subsubsection{Experimental Results}

Experimental results with a model ship are reported in Loria, Fossen and Panteley (1999). The experiments document that the nonlinear separation principle holds since the bias estimator manage to estimate a large off-set in the ship model. The physical bias is generated by using a ducted fan producing wind loads.

\section{Weather Optimal Passive DP}

In this section a new principle for weather optimal positioning control is presented. This is based on Fossen and Strand (1999).

\subsection{Introduction}

Conventional DP systems for ships and free-floating rigs are usually designed for station-keeping by specifying a desired constant position $\left(x_{d}, y_{d}\right)$ and a desired constant heading angle $\psi_{d}$. In order to minimize the fuel consumption of the ship the desired heading $\psi_{d}$ should in many operations be chosen such that the yaw moment is zero. For vessels with port/starboard symmetry this means that the mean environmental force due to wind, waves and currents will attack through the center line of the vessel in order to produce a zero yaw moment. Unfortunately, it is impossible to measure or compute the direction of the mean environmental force with sufficient accuracy. Hence, 
the desired heading $\psi_{d}$ is usually taken to be the measurement of the mean wind direction which can be easily measured. In the North Sea, however, this can result in off-sets up to 30 degrees from the true mean direction of the total environmental force. The main reason for this is the unmeasured current force component. Hence, the DP system can be operated under highly non-optimal conditions if fuel saving is the issue. A small off-set in the optimal heading angle will result in a large use of thrust.

Another approach for computing the weather optimal heading $\psi_{d}$ is to monitor the resulting thruster forces in the $x$ - and $y$-directions. Hence, the bow of the ship can be turned in one direction until the thruster force in the $y$-direction approaches zero. This method is appealing but the main catch in doing this is that the total resulting thruster forces in the $x$ - and $y$-directions have to be computed since there are no sensors doing this job directly. The sensors only measure the angular speed and pitch angle of the propellers. Hence, the thrust for each propeller must be computed by using a model of the thruster characteristic resulting in a pretty rough estimate of the total thruster force in each direction. Another principle, proposed by Pinkster and Nienhuis (1996), is to control the $x$ - and $y$-positions using a PID feedback controller, in addition to feedback from the yaw velocity, such that the vessel tends towards the optimal heading. This principle, however, requires that the rotation point of the vessel is located in a certain distance fore of the centre of gravity, or even fore of the bow, and it also puts restrictions on the thruster configuration and the number of thrusters installed.

This section describes the design of a new concept for weather optimal positioning control (WOPC) of ships and free-floating rigs, see Fossen and Strand (1999) and Strand (1999). The control objective is that the vessel heading should adjust automatically to the mean environmental disturbances (wind, waves and currents) such that a minimum amount of energy is used in order to save fuel and reduce $\mathrm{NO}_{x} / \mathrm{CO}_{x}$-emissions without using any environmental sensors. This is particularly useful for FPSOs which can be located at the same position for years. Also DP operated supply vessels which must keep their position for days in loading/offloading operations have a great WOPC fuel saving potential. An extension of WOPC to tracking control of ships in transit is found in Berge (1999). Other useful references on nonlinear tracking control are Berge and Fossen (1998), and Godhavn, Fossen and Berge (1998).

Nonlinear and adaptive backstepping designs are used to derive the WOPC system. The concept of WOPC can also be implemented by using other control methods, e.g. feedback linearization.

The key feature of the WOPC is that no information about the environmental disturbances is required. This is important since the mean environmental disturbances acting on a floating vessel cannot be accurately measured or computed. We show that the ship can be exponentially stabilized on a circle arc with constant radius by letting the bow of the ship point towards the origin of the circle. In order to maintain a fixed position at the same time, a translatory circle center control law is designed. Moreover, the circle center is translated on-line such that the Cartesian position is constant while the bow of the ship is automatically turned up against the mean environmental force (weather-vaning). This approach is motivated by a pendulum in the gravity field where gravity is the unmeasured quantity. The circular motion of the controlled ship where the mean environmental force can be interpreted as an unknown force field can be programmed to behave as a pendulum in the gravity field, see Figure 18. 

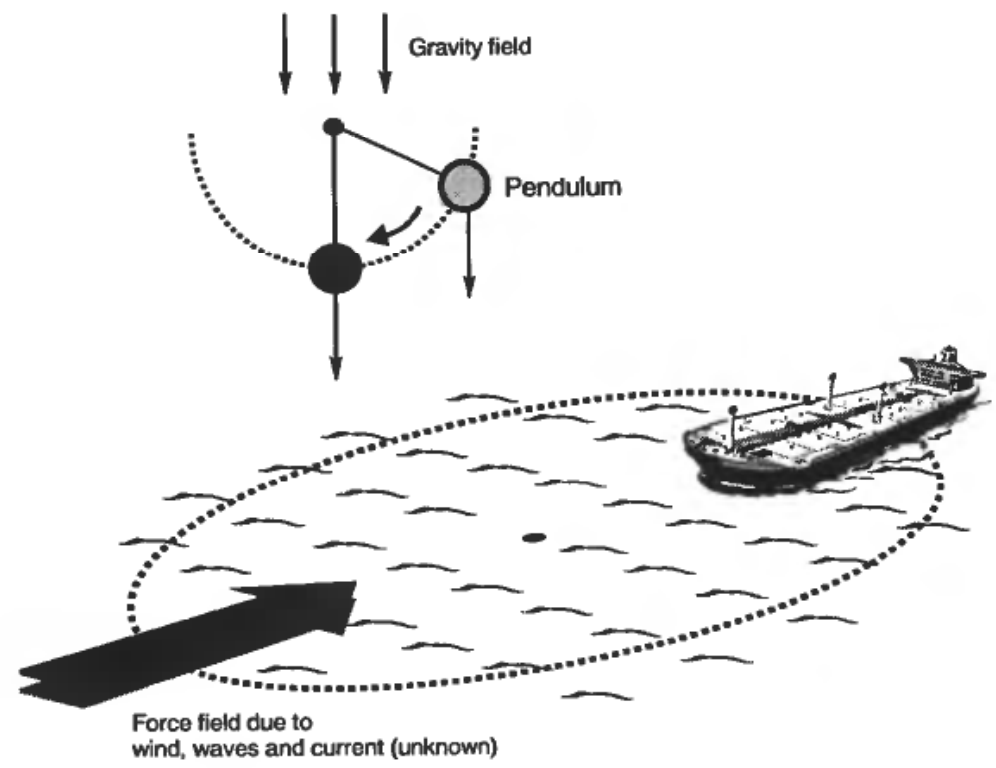

Figure 18. Weather optimal positioning principle: Equivalent to a pendulum in the gravity field, where gravity is the unmeasured quantity.

\subsection{Mathematical Modeling}

\subsubsection{Ship Model}

In this section we consider a low-speed ship model in $3 \mathrm{DOF}$ :

$$
\begin{aligned}
\mathbf{M} \dot{v}+\mathbf{C}(\boldsymbol{v}) \boldsymbol{v}+\mathbf{D}(\boldsymbol{v}) \boldsymbol{v} & =\boldsymbol{\tau}+\mathbf{w} \\
\dot{\boldsymbol{j}} & =\mathbf{J}(\psi) \boldsymbol{v}
\end{aligned}
$$

where the Earth-fixed position $(x, y)$ and heading $\psi$ is represented by $\boldsymbol{\eta}=[x, y, \psi]^{T}$ and the vessel-fixed vessel velocities are represented by $v=[u, v, r]^{T}$. The origin of the vessel-fixed frame $X Y Z$ is located at the vessel center line in a distance $x_{G}$ from the center of gravity. From the low-speed assumption, which is applied in the Lyapunov stability analysis, $\mathbf{M}=\mathbf{M}^{T}>0, \dot{\mathbf{M}}=\mathbf{0}$ and $\mathbf{D}(v)>0, \forall v \in \mathbb{R}^{3} . \tau \in \mathbb{R}^{3}$ is the control vector of forces in surge and sway and moment in yaw provided by the propulsion system. Unmodeled external forces and moment due to wind, currents and waves are lumped together into a vessel-fixed disturbance vector $w \in \mathbb{R}^{3}$.

\subsubsection{Polar Coordinates}

The Cartesian coordinates $(x, y)$ is related to the polar coordinates by:

$$
\begin{aligned}
& x=x_{0}+\rho \cos \gamma \\
& y=y_{0}+\rho \sin \gamma
\end{aligned}
$$

where $\left(x_{0}, y_{0}\right)$ is the origin of a circle with radius $\rho$ and polar angle $\gamma$ :

$$
\rho=\sqrt{\left(x-x_{0}\right)^{2}+\left(y-y_{0}\right)}
$$




$$
\gamma=\operatorname{atan} 2\left(\left(y-y_{0}\right),\left(x-x_{0}\right)\right)
$$

Time differentiation of (150) and (151), yields:

$$
\begin{aligned}
& \dot{x}=\dot{x}_{0}+\dot{\rho} \cos \gamma-\rho \sin \gamma \dot{\gamma} \\
& \dot{y}=\dot{y}_{0}+\dot{\rho} \sin \gamma+\rho \cos \gamma \dot{\gamma}
\end{aligned}
$$

Define the state vectors

$$
\begin{gathered}
\mathbf{p}_{0} \triangleq\left[x_{0}, y_{0}\right]^{T} \\
\mathbf{x} \triangleq[\rho, \gamma, \psi]^{T}
\end{gathered}
$$

From (154) and (155) a new kinematic relationship can be written in terms of the vectors $\mathbf{p}_{0}$ and $\mathbf{x}$ as:

$$
\dot{\boldsymbol{\eta}}=\mathbf{J}(\gamma) \mathbf{H}(\rho) \dot{\mathbf{x}}+\mathbf{L} \dot{\mathbf{p}}_{0}
$$

where

$$
\mathbf{H}(\rho)=\left[\begin{array}{lll}
1 & 0 & 0 \\
0 & \rho & 0 \\
0 & 0 & 1
\end{array}\right], \quad \mathbf{L}=\left[\begin{array}{ll}
1 & 0 \\
0 & 1 \\
0 & 0
\end{array}\right]
$$

From (158) the Cartesian kinematics in (149) can be replaced by a differential equation for the polar coordinates:

$$
\dot{\mathbf{x}}=\mathbf{T}(\mathbf{x}) \boldsymbol{v}-\mathbf{T}(\mathbf{x}) \mathbf{R}^{T}(\psi) \mathbf{L} \dot{\mathbf{p}}_{0}
$$

where

$$
\begin{aligned}
\mathbf{T}(\mathbf{x}) & \triangleq \mathbf{H}^{-1}(\rho) \mathbf{J}^{T}(\gamma) \mathbf{J}(\psi) \\
& =\mathbf{H}^{-1}(\rho) \mathbf{J}^{T}(\gamma-\psi)
\end{aligned}
$$

Note that the conversion between Cartesian and polar coordinates is only a local diffeomorphism, since the radius must be kept larger than a minimum value, i.e. $\rho>\rho_{\min }>0$ in order to avoid the singular point $\rho=0$.

\subsubsection{Ship Model Transformation}

The ship model (149) can be represented by polar coordinates by using (160) and substituting

$$
\begin{aligned}
& v=\mathbf{T}^{-1}(\mathbf{x}) \dot{\mathbf{x}}+\mathbf{J}^{T} \mathbf{L} \dot{\mathbf{p}}_{0} \\
& \dot{\boldsymbol{v}}=\mathbf{T}^{-1}(\mathbf{x}) \ddot{\mathbf{x}}+\dot{\mathbf{T}}^{-1}(\mathbf{x}) \dot{\mathbf{x}}+\mathbf{J}^{T} \mathbf{L} \ddot{\mathbf{p}}_{0}+\dot{\mathbf{J}}^{T} \mathbf{L} \dot{\mathbf{p}}_{0},
\end{aligned}
$$

such that:

$$
\begin{gathered}
\mathbf{M} \dot{v}+\mathbf{C}(v) v+\mathbf{D}(v) v=\tau+\mathbf{w} \\
\hat{v} \rho>0
\end{gathered}
$$

$$
\mathbf{M}_{x}(\mathbf{x}) \ddot{\mathbf{x}}+\mathbf{C}_{x}(v, \mathbf{x}) \dot{\mathbf{x}}+\mathbf{D}_{x}(v, \mathbf{x}) \dot{\mathbf{x}}=\mathbf{T}^{-T} \mathbf{q}\left(v, \mathbf{x}, \dot{\mathbf{p}}_{0}, \ddot{\mathbf{p}}_{0}\right)+\mathbf{T}^{-T} \tau+\mathbf{T}^{-T} \mathbf{w}
$$


where

$$
\begin{aligned}
\mathbf{M}_{x}(\mathbf{x}) & =\mathbf{T}^{-T}(\mathbf{x}) \mathbf{M T} \mathbf{T}^{-1}(\mathbf{x}) \\
\mathbf{C}_{x}(v, \mathbf{x}) & =\mathbf{T}^{-T}(\mathbf{x})\left(\mathbf{C}(v)-\mathbf{M} \mathbf{T}^{-1}(\mathbf{x}) \dot{\mathbf{T}}(\mathbf{x})\right) \mathbf{T}^{-1}(\mathbf{x}) \\
\mathbf{D}_{x}(v, \mathbf{x}) & =\mathbf{T}^{-T}(\mathbf{x}) \mathbf{D}(v) \mathbf{T}^{-1}(\mathbf{x}) \\
\mathbf{q}\left(v, \mathbf{x}, \dot{\mathbf{p}}_{0}, \ddot{\mathbf{p}}_{0}\right) & =\mathbf{M} \mathbf{J}^{T}(\psi) \mathbf{L} \ddot{\mathbf{p}}_{0}+\mathbf{M} \dot{\mathbf{J}}^{T}(\psi) \mathbf{L} \dot{\mathbf{p}}_{0}+[\mathbf{C}(v)+\mathbf{D}(v)] \mathbf{J}^{T}(\psi) \mathbf{L} \dot{\mathbf{p}}_{0}
\end{aligned}
$$

where $\mathbf{M}_{x}(\mathbf{x}), \mathbf{C}_{x}(\boldsymbol{v}, \mathbf{x})$ and $\mathbf{D}_{x}(\boldsymbol{v}, \mathbf{x})$ can be shown to satisfy:

$$
\begin{aligned}
\mathbf{M}_{x}(\mathbf{x}) & =\mathbf{M}_{x}^{T}(\mathbf{x})>0, & & \forall \mathbf{x} \\
\mathbf{D}_{x}(v, \mathbf{x}) & >0, & & \forall \mathbf{x}
\end{aligned}
$$

The ship dynamics does also satisfy the skew-symmetric property:

$$
\mathbf{z}^{T}\left(\dot{\mathbf{M}}_{x}-2 \mathbf{C}_{x}\right) \mathbf{z}=0, \quad \forall \mathbf{z}, \mathbf{x}
$$

The expression for $\mathbf{T}$ can be written as:

$$
\begin{aligned}
\dot{\mathbf{T}}(\mathbf{x}) & =\dot{\mathbf{H}}^{-1}(\rho) \mathbf{J}^{T}(\gamma-\psi)-\mathbf{H}^{-1}(\rho) \dot{\mathbf{J}}^{T}(\gamma-\psi) \\
& =-\dot{\rho} \Pi(\rho)-(\dot{\gamma}-\dot{\psi}) \mathbf{H}^{-1}(\rho) \mathbf{S}_{o} \mathbf{J}(\gamma-\psi)
\end{aligned}
$$

where:

$$
\Pi(\rho)=\left[\begin{array}{ccc}
0 & 0 & 0 \\
0 & \frac{1}{\rho^{2}} & 0 \\
0 & 0 & 0
\end{array}\right]
$$

and $\mathbf{J}(\alpha)$ satisfies:

$$
\dot{J}(\alpha)=\dot{\alpha} \mathbf{J}(\alpha) \mathbf{S}_{o}
$$

\subsubsection{Disturbance Modeling}

The steady-state low-frequency motion of the ship and also the ship's equilibrium position depend on the unknown environmental loads acting on the vessel. Let the environmental loads due to wind, waves and currents be represented by:

- a slowly-varying mean force $F_{e}$ which attacks the ship in a point $\left(l_{x}, l_{y}\right)$ in bodyfixed coordinates.

- a slowly-varying mean direction $\beta_{e}$, relative to the Earth-fixed frame, see Figure 19.

The term slowly-varying simply states that the mean environmental forces are slow compared to the vessel kinematics and dynamics. The slowly-varying terms include 2nd-order wave-induced disturbances (wave drift), currents and mean wind forces. The WF motion is assumed to be filtered out of the measurements by using a wave filter.

Since there are no sensors which can be used to measure $\left(F_{e}, \beta_{e}\right)$ and $\left(l_{x}, l_{y}\right)$ with sufficient accuracy it is impossible to use feedforward from the environmental disturbances. This motivates the following assumptions:

A1: The unknown mean environmental force $F_{e}$ and its direction $\beta_{e}$ are assumed to be constant or at least slowly-varying.

A2: The unknown attack point $\left(l_{x}, l_{y}\right)$ is constant for each constant $F_{e}$. 


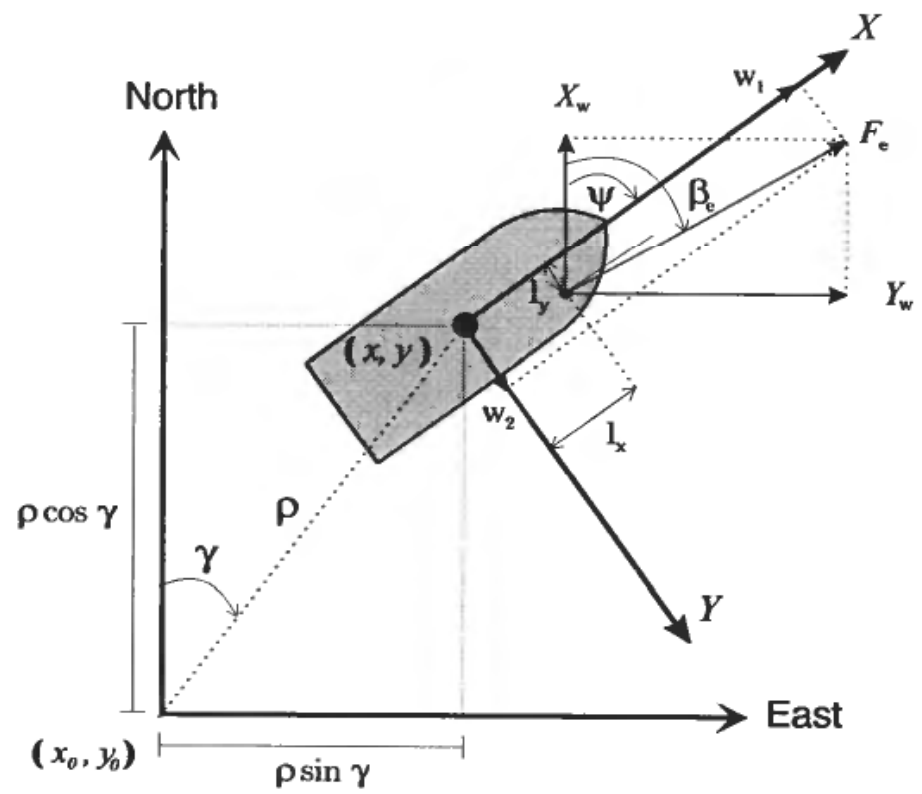

Figure 19. Environmental force $F_{e}$ decomposed into the components $w_{1}$ and $w_{2}$.

Discussion: These are good assumptions since the ship control system is only supposed to counteract the slowly-varying motion components of the environmental disturbances. From Figure 19 the body-fixed environmental load vector $\mathbf{w} \in \mathscr{R}^{3}$ can be expressed as:

$$
\mathbf{w}=\left[\begin{array}{c}
w_{1}(\psi) \\
w_{2}(\psi) \\
w_{3}(\psi)
\end{array}\right]=\left[\begin{array}{c}
F_{e} \cos \left(\beta_{e}-\psi\right) \\
F_{e} \sin \left(\beta_{e}-\psi\right) \\
l_{x} F_{e} \sin \left(\beta_{e}-\psi\right)-l_{y} F_{e} \cos \left(\beta_{e}-\psi\right)
\end{array}\right]
$$

Notice that the environmental loads vary with the heading angle $\psi$ of the ship. Moreover:

$$
\begin{aligned}
& F_{e}=\sqrt{w_{1}^{2}+w_{2}^{2}} \\
& \beta_{e}=\psi+\tan ^{-1}\left(w_{2} / w_{1}\right)
\end{aligned}
$$

The environmental forces $X_{w}$ and $Y_{w}$ with attack point $\left(l_{x}, l_{y}\right)$ are shown in Figure 19. It should be noted that the attach point $l_{x}=l_{x}(\psi)$ and $l_{y}=l_{y}(\psi)$ will also change with the yaw angle $\psi$. This relationship will be complicated function of the hull and superstructure geometries.

\subsection{Weather Optimal Control Objectives}

The weather optimal control objectives make use of the following definitions (Fossen and Strand (1999)):

Definition 5.1 (Weather Optimal Heading) The weather optimal heading angle $\psi_{\mathrm{opt}}$ is given by the equilibrium state where the yaw moment $w_{3}\left(\psi_{\mathrm{opt}}\right)=0$ at the same time as the bow of the ship is turned up against weather (mean environmental disturbances), that is $w_{2}\left(\psi_{\mathrm{opt}}\right)=0$. This implies that the moment arms $l_{x}\left(\psi_{\mathrm{opt}}\right)=$ constant and $l_{y}\left(\psi_{\mathrm{opt}}\right)=0$, and: 


$$
\mathbf{w}\left(\psi_{\mathrm{opt}}\right)=\left[\begin{array}{l}
w_{1}\left(\psi_{\mathrm{opt}}\right) \\
w_{2}\left(\psi_{\mathrm{opt}}\right) \\
w_{3}\left(\psi_{\mathrm{opt}}\right)
\end{array}\right]=\left[\begin{array}{c}
-F_{e} \\
0 \\
0
\end{array}\right]
$$

Hence, the mean environmental force attacks the ship in the bow (minimum drag coefficient for water and wind loads).

Definition 5.2 (Weather Optimal Positioning) Weather optimal positioning (stationkeeping) is defined as the equilibrium state where

$$
w_{1}\left(\psi_{\text {opt }}\right)=-F_{e}, w_{2}\left(\psi_{\text {opt }}\right)=w_{3}\left(\psi_{\text {opt }}\right)=l_{y}\left(\psi_{\text {opt }}\right)=0
$$

(weather optimal heading) and the position $(x, y)=\left(x_{d}, y_{d}\right)$ is kept constant.

These definitions motivates the following two control objectives:

O1: Weather Optimal Heading Control (WOHC): This is obtained by restricting the ship to move on a circle with constant radius $\rho=\rho_{d}$ and at the same time force the ship's bow to point towards the center of the circle until the weather optimal heading angle $\psi=\psi_{\text {opt }}$ is reached, see Figure 20. An analogy to this is a pendulum in gravity field, see Figure 18 . The position $(x, y)=\left(x_{0}+\rho \cos \gamma, y_{0}+\rho \sin \gamma\right)$ will vary until the weather optimal heading angle is reached. This is obtained by specifying the control objective in polar coordinates according to:

$$
\begin{gathered}
\rho_{d}=\text { constant } \\
\dot{\gamma}_{d}=0
\end{gathered}
$$

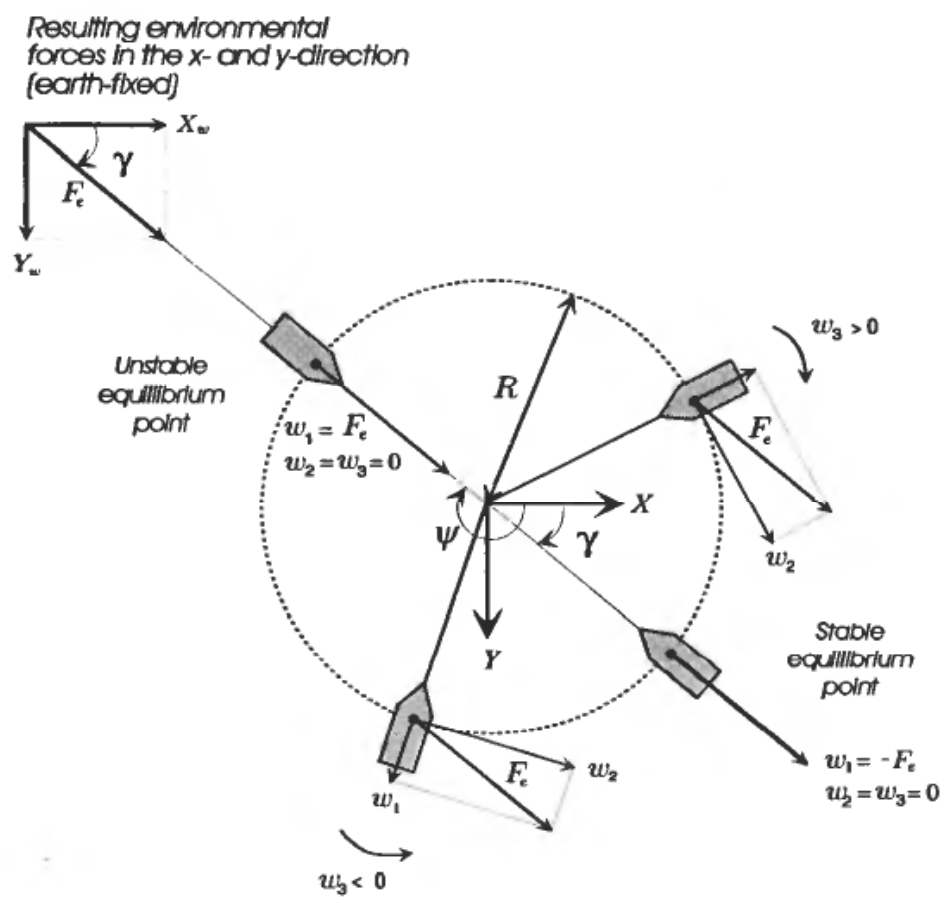

Figure 20. Principle for weather optimal heading control (WOHC). 


$$
\psi_{d}=\pi+\gamma .
$$

Discussion: The requirement $\rho_{d}=$ constant implies that the ship moves on a circle with constant radius. The second requirement $\dot{\gamma}_{d}=0$ implies that the tangential speed $\rho \dot{\gamma}$ is kept small while the last requirement $\psi_{d}=\pi+\gamma$ ensures that the ship's bow points towards the center of the circle.

O2: Weather Optimal Positioning Control (WOPC): In order to maintain a fixed Earth-fixed position $(x, y)=\left(x_{d}, y_{d}\right)$, the circle center $\mathbf{p}_{0}=\left[x_{0}, y_{0}\right]^{T}$ must be moved simultaneously as Control Objective $\mathrm{Ol}$ is satisfied. This is referred to as translatory circle center control.

We will derive a nonlinear and adaptive backstepping controller which satisfies $\mathrm{O} 1$ and $\mathrm{O} 2$.

\subsection{Nonlinear and Adaptive Control Design}

In this section a positioning controller is presented by using the polar coordinate representation of the ship dynamics. Backstepping is used to derive the feedback controller (Krstic et al. (1995)). The control law will be derived in 3 successive steps:

1. Nonlinear backstepping (PD-control): the ship is forced to move on a circle arc with desired radius $\rho_{d}$, with minimum tangential velocity $\rho \dot{\gamma}$ and desired heading $\psi_{d}$.

2. Adaptive backstepping (PID-control): this is necessary to compensate for the unknown environmental force $F_{e}$.

3. Translatory circle center control: the circle center $\left(x_{0}, y_{0}\right)$ is translated such that the ship maintains a constant position $\left(x_{d}, y_{d}\right)$ even though it is moving on a virtual circle arc. Hence, the Captain of the ship will only notice that the ship is rotating a yaw angle $\psi$ about a constant position $\left(x_{d}, y_{d}\right)$ until the weather optimal heading $\psi_{\mathrm{opt}}$ is reached.

\subsubsection{Nonlinear Backstepping (PD-Control)}

A general positioning controller is derived by using vectorial backstepping. The tracking objective is specified in polar coordinates by the smooth reference trajectory $\mathbf{x}_{d}=\left[\rho_{d}, \gamma_{d}, \psi_{d}\right]^{T} \in C^{3}$ where:

$$
\mathbf{x}_{d}, \dot{\mathbf{x}}_{d}, \ddot{\mathbf{x}}_{d} \in \mathscr{L}_{\infty}
$$

Since the transformed system (164) is of order 2, backstepping is performed in two vectorial steps resulting in a nonlinear PD-control law. First, we define a virtual reference trajectory as:

$$
\dot{\mathbf{x}}_{r} \triangleq \dot{\mathbf{x}}_{d}-\Lambda \mathbf{z}_{1},
$$

where $\mathbf{z}_{1}=\mathbf{x}-\mathbf{x}_{d}$ is the Earth-fixed tracking error and $\Lambda>0$ is a diagonal design matrix. Furthermore, let $\mathbf{z}_{2}$ denote a measure of tracking defined according to:

$$
\mathbf{z}_{2} \triangleq \dot{\mathbf{x}}-\dot{\mathbf{x}}_{r}=\dot{\mathbf{z}}_{1}+\Lambda \mathbf{z}_{1}
$$

From (181), the following expressions are obtained:

$$
\dot{\mathbf{x}}=\mathbf{z}_{2}+\dot{\mathbf{x}}_{r}
$$




$$
\ddot{\mathbf{x}}=\dot{\mathbf{z}}_{2}+\ddot{\mathbf{x}}_{r}
$$

This implies that the vessel model (164) can be expressed in terms of $\mathbf{z}_{2}, \dot{\mathbf{x}}_{r}$ and $\ddot{\mathbf{x}}_{r}$ as:

$$
\mathbf{M}_{x} \dot{\mathbf{z}}_{2}+\mathbf{C}_{x} \mathbf{z}_{2}+\mathbf{D}_{x} \mathbf{z}_{2}=\mathbf{T}^{-T} \tau+\mathbf{T}^{-T} \mathbf{q}(\cdot)-\mathbf{M}_{x} \ddot{\mathbf{x}}_{r}-\mathbf{C}_{x} \dot{\mathbf{x}}_{r}-\mathbf{D}_{w} \dot{\mathbf{x}}_{r}+\mathbf{T}^{-T} \mathbf{W}
$$

Step 1: Let $\mathbf{z}_{1}$ be the first error variable, which from (181) has the dynamics:

$$
\dot{\mathbf{z}}_{1}=-\Lambda \mathbf{z}_{1}+\mathbf{z}_{2}
$$

A Lyapunov function candidate (LFC) for the first step is:

$$
\begin{gathered}
V_{1}=\frac{1}{2} \mathbf{z}_{1}^{T} \mathbf{K}_{p} \mathbf{z}_{1} \\
\dot{\mathrm{V}}_{1}=-\mathbf{z}_{1}^{T} \mathbf{K}_{p} \Lambda \mathbf{z}_{1}+\mathbf{z}_{1}^{T} \mathbf{K}_{p} \mathbf{z}_{2}
\end{gathered}
$$

where $\mathbf{K}_{p}=\mathbf{K}_{p}^{\mathrm{T}}>0$ is a constant design matrix.

Step 2: In the second step we choose a LFC motivated by the "pseudo" kinetic energy, that is:

$$
V_{2}=V_{1}+\frac{1}{2} \mathbf{z}_{2}^{T} \mathbf{M}_{x} \mathbf{z}_{2}, \quad \mathbf{M}_{x}=\mathbf{M}_{x}^{T}>0
$$

Time differentiation of $V_{2}$ along the trajectories of $\mathbf{z}_{1}$ and $\mathbf{z}_{2}$ yields:

$$
\dot{V}_{2}=\dot{V}_{1}+\mathbf{z}_{2}^{T} \mathbf{M}_{x} \dot{\mathbf{z}}_{2}+\frac{1}{2} \mathbf{z}_{2}^{T} \dot{\mathbf{M}}_{x} \mathbf{z}_{2}
$$

which by substitution of (187) and (184) yields

$$
\begin{aligned}
\dot{V}_{2}= & -\mathbf{z}_{1}^{T} \mathbf{K}_{p} \Lambda \mathbf{z}_{1}+\frac{1}{2} \mathbf{z}_{2}^{T}\left(\dot{\mathbf{M}}_{x}-2 \mathbf{C}_{x}\right) \mathbf{z}_{2}-\mathbf{z}_{2}^{T} \mathbf{D}_{x} \mathbf{z}_{2}+\mathbf{z}_{2}^{T} \mathbf{T}^{-T} \mathbf{w} \\
& +\mathbf{z}_{2}^{T}\left(\mathbf{K}_{p} \mathbf{z}_{1}+\mathbf{T}^{-T} \tau+\mathbf{T}^{-T} \mathbf{q}(\cdot)-\mathbf{M}_{x} \ddot{\mathbf{x}}_{r}-\mathbf{C}_{x} \dot{\mathbf{x}}_{r}-\mathbf{D}_{x} \dot{\mathbf{x}}_{r}\right)
\end{aligned}
$$

By using the property (169) and choosing the nonlinear PD-control law as:

$$
\mathbf{T}^{-T} \boldsymbol{\tau}=\mathbf{M}_{x} \ddot{\mathbf{x}}_{r}+\mathbf{C}_{x} \dot{\mathbf{x}}_{r}+\mathbf{D}_{x} \dot{\mathbf{x}}_{r}-\mathbf{K}_{p} \mathbf{z}_{1}-\mathbf{K}_{d} \mathbf{z}_{2}-\mathbf{T}^{-T} \mathbf{q}(\cdot)
$$

where $\mathbf{K}_{d}>0$ is a strictly positive design matrix, we finally get:

$$
\dot{V}_{2}=-\mathbf{z}_{1}^{T} \mathbf{K}_{p} \Lambda \mathbf{z}_{1}-\mathbf{z}_{2}^{T}\left(\mathbf{K}_{d}+\mathbf{D}_{x}\right) \mathbf{z}_{2}+\mathbf{z}^{T} \mathbf{T}^{-T} \mathbf{w}
$$

Notice that the dissipative term $\mathbf{z}_{2}^{T} \mathbf{D}_{x} \mathbf{z}_{2}>0, \forall \mathbf{z}_{2} \neq \mathbf{0}$ is exploited in the design as it appears in the expression for $\dot{V}_{2}$. With the control law (191) the closed-loop dynamics becomes:

$$
\mathbf{M}_{x} \dot{\mathbf{z}}_{2}+\left(\mathbf{C}_{x}+\mathbf{D}_{x}+\mathbf{K}_{d}\right) \mathbf{z}_{2}+\mathbf{K}_{p} \mathbf{z}_{1}=\mathbf{T}^{-T} \mathbf{w}
$$

The error dynamics of the resulting system becomes non-autonomous since:

$$
\left[\begin{array}{cc}
\mathbf{K}_{p} & \mathbf{0}_{3 \times 3} \\
\mathbf{0}_{3 \times 3} & \mathbf{M}_{x}
\end{array}\right]\left[\begin{array}{c}
\dot{\mathbf{z}}_{1} \\
\dot{\mathbf{z}}_{2}
\end{array}\right]=-\left[\begin{array}{cc}
\mathbf{K}_{p} \Lambda & \mathbf{0}_{3 \times 3} \\
\mathbf{0}_{3 \times 3} & \mathbf{C}_{x}+\mathbf{D}_{x}+\mathbf{K}_{d}
\end{array}\right]\left[\begin{array}{l}
\mathbf{z}_{1} \\
\mathbf{z}_{2}
\end{array}\right]
$$




$$
\begin{gathered}
+\left[\begin{array}{cc}
\mathbf{0}_{3 \times 3} & \mathbf{K}_{p} \\
-\mathbf{K}_{p} & \mathbf{0}_{3 \times 3}
\end{array}\right]\left[\begin{array}{l}
\mathbf{z}_{1} \\
\mathbf{z}_{2}
\end{array}\right]+\left[\begin{array}{c}
\mathbf{0}_{3 \times 1} \\
\mathbf{T}^{-T}
\end{array}\right] \mathbf{w} \\
\hat{\Downarrow} \\
\mathscr{H}(\mathbf{x}) \dot{\mathbf{z}}=-\mathscr{K}(\mathbf{x}, v) \mathbf{z}+\mathscr{S} \mathbf{z}+\overline{\mathscr{B}}(\mathbf{x}) \mathbf{w}
\end{gathered}
$$

where the different matrices are defined as:

$$
\begin{aligned}
\mathscr{M}(\mathbf{x}) & =\mathscr{H}^{T}(\mathbf{x})=\left[\begin{array}{cc}
\mathbf{K}_{p} & \mathbf{0}_{3 \times 3} \\
\mathbf{0}_{3 \times 3} & \mathbf{M}_{\mathbf{x}}(\mathbf{x})
\end{array}\right] \\
\mathscr{K}(\mathbf{x}, v) & =\left[\begin{array}{cc}
\mathbf{K}_{p} \Lambda & \mathbf{0}_{3 \times 3} \\
\mathbf{0}_{3 \times 3} & \mathbf{C}_{x}(\mathbf{x}, v)+\mathbf{D}_{\mathbf{x}}(\mathbf{x}, v)+\mathbf{K}_{d}
\end{array}\right]>0 \\
\mathscr{S} & =-\mathscr{S}^{T}=\left[\begin{array}{cc}
\mathbf{0}_{3 \times 3} & \mathbf{K}_{p} \\
-\mathbf{K}_{p} & \mathbf{0}_{3 \times 3}
\end{array}\right] \\
\overline{\mathscr{B}}(\mathbf{x}) & =\left[\begin{array}{c}
\mathbf{0}_{3 \times 1} \\
\mathbf{T}^{-T}(\mathbf{x})
\end{array}\right]
\end{aligned}
$$

In the absence of disturbances, $\mathbf{w} \equiv \mathbf{0}$, the origin $\mathbf{z}=\mathbf{0}$ is uniformly locally exponentially stable (ULES) according to Lyapunov. Global results cannot be achieved due to the local diffeomorphism between the Cartesian and polar coordinates, hat is the transformation matrix $\mathbf{T}(\mathbf{x})$ is singular for $\rho=0$.

With disturbances $\mathbf{w} \neq \mathbf{0}$, the closed-loop system is ISS. In the next section, we will show how adaptive back-stepping (backstepping with integral action) can be used to obtain ULES for the case of a non-zero disturbance vector $\mathbf{w} \neq \mathbf{0}$.

\subsubsection{Adaptive Backstepping (PID-Control)}

If the disturbance vector $w$ have a non-zero mean, this will result in a steady-state offset when using the nonlinear PD-controller. Since the ship is restricted to move on a circle arc where $\mathbf{w}$ can be viewed as a force field there will be a stable and unstable equilibrium point on the circle arc (similar to a pendulum in the gravity field). The stable equilibrium point is, see Figure 20:

$$
\mathbf{w}=\phi F_{e}=\left[\begin{array}{c}
-1 \\
0 \\
0
\end{array}\right] F_{e}
$$

Since the disturbance $F_{e}$ is assumed to be slowly-varying, we can apply adaptive backstepping to obtain integral effect in the system. Thus. in the analysis it will be assumed that

$$
\dot{F}_{e}=0
$$

Let the estimate of $F_{e}$ be denoted as $\hat{F}_{e}$, and $\tilde{F}=\hat{F}_{e}-F_{e}$. An additional step in the derivation of the backstepping control must be performed in order to obtain an adaptive update law for $\hat{F}_{e}$. Moreover: 
Step 3: The adaptive update law is found by adding the squared parameter estimation error to $V_{2}$. Moreover:

$$
V_{3}=V_{2}+\frac{1}{2 \sigma} \tilde{F}_{e}^{2}, \sigma>0
$$

where

$$
\dot{V}_{3}=\dot{V}_{2}+\frac{1}{\sigma} \dot{\tilde{F}}_{e} \tilde{F}_{e}
$$

The nonlinear control law (191) is modified to:

$$
\tau=\mathbf{T}^{T}\left(\mathbf{M}_{x} \ddot{\mathbf{x}}_{r}+\mathbf{C}_{x} \dot{\mathbf{x}}_{r}+\mathbf{D}_{x} \dot{\mathbf{x}}_{r}-\mathbf{K}_{p} \mathbf{z}_{1}-\mathbf{K}_{d} \mathbf{z}_{2}\right)-\mathbf{q}(\cdot)-\phi \hat{F}_{e}
$$

where the last term $\phi \hat{F}_{e}$ provides integral action. Hence, the $\mathbf{z}_{2}$-dynamics becomes:

$$
\mathbf{M}_{x} \dot{\mathbf{z}}_{2}+\left(\mathbf{C}_{x}+\mathbf{D}_{x}+\mathbf{K}_{d}\right) \mathbf{z}_{2}+\mathbf{K}_{p} \mathbf{z}_{1}=-\mathbf{T}^{-T} \phi \tilde{F}_{e}
$$

This implies that:

$$
\begin{aligned}
\dot{V}_{3} & =-\mathbf{z}_{1}^{T} \mathbf{K}_{p} \Lambda \mathbf{z}_{1}-\mathbf{z}_{2}^{T}\left(\mathbf{K}_{d}+\mathbf{D}_{x}\right) \mathbf{z}_{2}-\mathbf{z}_{2}^{T} \mathbf{T}^{-T} \phi \tilde{F}_{e}+\frac{1}{\sigma} \dot{\tilde{F}}_{e} \tilde{F}_{e} \\
& =-\mathbf{z}_{1}^{T} \mathbf{K}_{p} \Lambda \mathbf{z}_{1}-\mathbf{z}_{2}^{T}\left(\mathbf{K}_{d}+\mathbf{D}_{x}\right) \mathbf{z}_{2}+\tilde{F}_{e}\left(-\boldsymbol{\phi}^{T} \mathbf{T}^{-1} \mathbf{z}_{2}+\frac{1}{\sigma} \dot{\tilde{F}}_{e}\right)
\end{aligned}
$$

The adaptive law $\dot{\tilde{F}}_{e}=\dot{\hat{F}}_{e}$ is chosen as:

$$
\dot{\hat{F}}_{e}=\sigma \phi^{T} \mathbf{T}^{-1} \mathbf{z}_{2}, \sigma>0
$$

such that

$$
\dot{V}_{3}=-\mathbf{z}_{1}^{T} \mathbf{K}_{p} \Lambda \mathbf{z}_{1}-\mathbf{z}_{2}^{T}\left(\mathbf{K}_{d}+\mathbf{D}_{x}\right) \mathbf{z}_{2} \leqslant 0
$$

The non-autonomous error dynamics for the adaptive backstepping controller can be written:

$$
\begin{aligned}
\mathscr{H}(\mathbf{x}) \dot{\mathbf{z}} & =[-\mathscr{K}(\mathbf{x}, v)+\mathscr{S}] \mathbf{z}+\mathscr{B}(\mathbf{x}) \tilde{F}_{e} \\
\dot{\tilde{F}}_{\boldsymbol{e}} & =-\sigma \mathscr{B}^{T}(\mathbf{x}) \mathbf{z},
\end{aligned}
$$

where

$$
\mathscr{B}(\mathbf{x})=\left[\begin{array}{c}
\mathbf{0}_{3 \times 1} \\
-\mathbf{T}^{-T}(\mathbf{x}) \phi
\end{array}\right]
$$

In order to satisfy Control Objective $\mathrm{O} 1$ we must choose the controller gains according to:

$$
\mathbf{K}_{p}=\left[\begin{array}{ccc}
k_{p 1} & 0 & 0 \\
0 & 0 & 0 \\
0 & 0 & k_{p 3}
\end{array}\right], \mathbf{K}_{d}=\left[\begin{array}{ccc}
k_{d 1} & 0 & 0 \\
0 & k_{d 2} & 0 \\
0 & 0 & k_{d 3}
\end{array}\right], \Lambda=\left[\begin{array}{ccc}
\lambda_{1} & 0 & 0 \\
0 & 0 & 0 \\
0 & 0 & \lambda_{3}
\end{array}\right]
$$


Notice that $k_{p 2}=\lambda_{2}=0$. This implies that the ship is free to move on the circle arc with tangential velocity $\rho \dot{\gamma}$. The gain $k_{d 2}>0$ is used to increase the tangential damping (D-control) while the radius $\rho$ and heading $\psi$ are stabilized by using PD-control.

\section{Semi-Definite Matrices}

Since the controller gains $k_{p 2}$ and $\lambda_{2}$ are chosen to be zero, the matrices:

$$
\mathbf{K}_{p} \geqslant 0, \quad \Lambda \geqslant 0
$$

are only positive semi-definite resulting in a positive semi-definite $V_{3}$. Uniform local asymptotic stability $(U L A S)$ of the equilibrium $\left(\mathbf{z}, \tilde{F}_{e}\right)=(\mathbf{0}, 0)$ can, however, be proven since the system is input-to-state (ISS) stable. We therefore consider the error dynamics of a system with outputs $\left(\mathbf{z}_{1 r}, \mathbf{z}_{2}\right)$ where:

$$
\mathbf{z}_{1 r}=\mathbf{E z}_{1}
$$

where

$$
\mathbf{E}=\left[\begin{array}{lll}
1 & 0 & 0 \\
0 & 0 & 1
\end{array}\right]
$$

This implies that:

$$
\begin{aligned}
\dot{\mathbf{z}}_{1 r} & =-\mathbf{E} \Lambda \mathbf{z}_{1}+\mathbf{E} \mathbf{z}_{2} \\
& =-\left(\mathbf{E} \Lambda \mathbf{E}^{T}\right) \mathbf{z}_{1 r}+\mathbf{E} \mathbf{z}_{2}
\end{aligned}
$$

Notice that the last step is possible since the diagonal matrices $\Lambda=\operatorname{diag}\left\{\lambda_{1}, 0, \lambda_{3}\right\}$ satisfies:

$$
\Lambda \mathbf{E}^{T} \mathbf{z}_{1 r}=\Lambda \mathbf{z}_{1}
$$

Hence, the error dynamics (209)-(210) can be transformed to:

$$
\begin{aligned}
\mathscr{M}_{r}(\mathbf{x}) \dot{\mathbf{z}}_{r} & =\left[-\mathscr{K}_{r}(\mathbf{x}, \boldsymbol{v})+\mathscr{S}_{r}\right] \mathbf{z}_{r}+\mathscr{B}_{r}(\mathbf{x}) \tilde{F}_{e} \\
\dot{\tilde{F}}_{e} & =-\sigma \mathscr{B}_{r}^{T}(\mathbf{x}) \mathbf{z}_{r}
\end{aligned}
$$

where $\mathbf{z}_{r}=\left[\mathbf{z}_{1 r}^{T}, \mathbf{z}_{2}^{T}\right]^{T}$ and:

$$
\begin{aligned}
\mathscr{M}_{r}(\mathbf{x}) & =\mathscr{M}_{r}^{T}(\mathbf{x})=\left[\begin{array}{cc}
\left(\mathbf{E K}_{p} \mathbf{E}^{T}\right) & \mathbf{0}_{2 \times 3} \\
\mathbf{0}_{3 \times 2} & \mathbf{M}_{x}(\mathbf{x})
\end{array}\right] \\
\mathscr{K}_{r}(\mathbf{x}, v) & =\left[\begin{array}{cc}
\left(\mathbf{E K}_{p} \mathbf{E}^{T}\right)\left(\mathbf{E} \wedge \mathbf{E}^{T}\right) & \mathbf{0}_{2 \times 3} \\
\mathbf{0}_{3 \times 2} & \mathbf{C}_{x}(\mathbf{x}, v)+\mathbf{D}_{x}(\mathbf{x}, v)+\mathbf{K}_{d}
\end{array}\right]>0 \\
\mathscr{S}_{r} & =-\mathscr{S}_{r}^{T}=\left[\begin{array}{cc}
\mathbf{0}_{2 \times 2} & \mathbf{K}_{p} \mathbf{E} \\
-\mathbf{K}_{p} \mathbf{E}^{T} & \mathbf{0}_{3 \times 3}
\end{array}\right] \\
\mathscr{B}_{r}(\mathbf{x}) & =\left[\begin{array}{c}
\mathbf{0}_{2 \times 1} \\
\mathbf{T}^{-T}(\mathbf{x}) \phi
\end{array}\right]
\end{aligned}
$$

We have here used the fact that $\mathbf{K}_{p} \mathbf{E}^{T} \mathbf{z}_{1 r}=\mathbf{K}_{p} \mathbf{z}_{1}$ for $\mathbf{K}_{p}=\operatorname{diag}\left\{k_{p 1}, 0, k_{p 3}\right\}$. 


\section{Non-Autonomous Lyapunov Analysis}

Recall that the Lyapunov function $V_{3}$ is only semi-definite since $K_{p}$ is positive semi-definite. Since the system is ISS asymptotic output tracking is guaranteed by:

$$
\begin{aligned}
& V_{3 r}=\frac{1}{2}\left[\mathbf{z}_{1 r}^{T}\left(\mathbf{E K}_{p} \mathbf{E}^{T}\right) \mathbf{z}_{1 r}+\mathbf{z}_{2}^{T} \mathbf{M}_{x} \mathbf{z}_{2}+\frac{1}{\sigma} \tilde{F}_{e}^{2}\right]>0 \\
& \dot{V}_{3 r}=-\mathbf{z}_{1 r}^{T}\left(\mathbf{E K}_{p} \mathbf{E}^{T}\right)\left(\mathbf{E} \Lambda \mathbf{E}^{T}\right) \mathbf{z}_{1 r}-\mathbf{z}_{2}^{T}\left(\mathbf{K}_{d}+\mathbf{D}_{x}\right) \mathbf{z}_{2} \leqslant 0
\end{aligned}
$$

where $\mathbf{E K}_{p} \mathbf{E}^{T}>0$ and $\mathbf{E} \Lambda \mathbf{E}^{T}>0$. Hence, $\mathbf{z}_{1 r}, \mathbf{z}_{2}, \tilde{F}_{e} \in \mathscr{L}_{\infty}$. ULES of the equilibrium point $\left(\mathbf{z}_{1 r}, \mathbf{z}_{2}, \tilde{F}_{e}\right)=(\mathbf{0}, \mathbf{0}, 0)$ follows by using the stability theorem of Loria, Fossen and Teel (1999) for nonlinear non-autonomous systems where $V_{3}>0$ (positive definite) and $\dot{V}_{3} \leqslant 0$ (negative semi-definite). The reason that $\dot{V}_{3}$ is only negative semi-definite is that a negative term proportional to $-\tilde{F}_{e}^{2}$ is missing in the expression for $\dot{V}_{3}$.

We are now ready to state the main theorem of this section.

Theorem 5.1 (Main Result: ULES Weather Optimal Position Control) The equilibrium point $\left(\mathbf{z}_{1}, \mathbf{z}_{2}, \tilde{F}_{e}\right)=(\mathbf{0}, \mathbf{0}, 0)$ of the nonlinear system (218) and (219) with control law (204) and parameter adaptation law (207) is ULES.

Proof. See Fossen and Strand (1999).

\subsubsection{Translatory Circle Center Controller}

The adaptive backstepping controller of the previous section satisfies control objective $\mathrm{O} 1$, that is weather optimal heading control. Weather optimal position control, control objective $\mathrm{O} 2$, can be satisfied by moving the circle center $\mathbf{p}_{0}=\left[x_{0}, y_{0}\right]^{T}$ such that the ship maintains a constant position $\mathbf{p}=[x, y]^{T}$.

In order to meet the fixed position control objective, an update law for the circle center $\mathbf{p}_{0}$ must be derived. Recall that the Cartesian Earth-fixed position of the ship is given by:

$$
\mathbf{p}=\mathbf{L}^{T} \boldsymbol{\eta}
$$

where $\mathbf{L}$ is defined in (159). Let $\tilde{\mathbf{p}} \triangleq \mathbf{p}-\mathbf{p}_{d}$ denote the corresponding deviation from the desired position vector $\mathbf{p}_{d} \triangleq\left[x_{d}, y_{d}\right]^{T}$. The desired position can either be constant (regulation) or a smooth time-varying reference trajectory. The control law for translation of the circle center is derived by considering the following LFC:

$$
V_{p}=\frac{1}{2} \tilde{\mathbf{p}}^{T} \tilde{\mathbf{p}}
$$

where

$$
\dot{V}_{p}=\tilde{\mathbf{p}}^{T}\left(\dot{\mathbf{p}}-\dot{\mathbf{p}}_{d}\right)=\tilde{\mathbf{p}}^{T}\left(\mathbf{L}^{T} \dot{\boldsymbol{\eta}}-\dot{\mathbf{p}}_{d}\right)
$$

By using (158), $\mathbf{L}^{T} \mathbf{L}=\mathbf{I}_{2 \times 2}$ and $\dot{\mathbf{x}}=\mathbf{z}_{2}+\dot{\mathbf{x}}_{r}$ we get:

$$
\begin{aligned}
\dot{V}_{p} & =\tilde{\mathbf{p}}^{T}\left[\mathbf{L}^{T}\left(\mathbf{J}(\gamma) \mathbf{H}(\rho) \dot{\mathbf{x}}+\mathbf{L} \dot{\mathbf{p}}_{0}\right)-\dot{\mathbf{p}}_{d}\right] \\
& =\tilde{\mathbf{p}}^{T}\left(\dot{\mathbf{p}}_{0}-\dot{\mathbf{p}}_{d}+\mathbf{L}^{T} \mathbf{J}(\gamma) \mathbf{H}(\rho) \dot{\mathbf{x}}_{r}\right)+\tilde{\mathbf{p}}^{T} \mathbf{L}^{T} \mathbf{J}(\gamma) \mathbf{H}(\rho) \mathbf{z}_{2}
\end{aligned}
$$

Now, hy choosing the circle center update law as: 


$$
\dot{\mathbf{p}}_{0}=\dot{\mathbf{p}}_{d}-\mathbf{L}^{T} \mathbf{J}(\gamma) \mathbf{H}(\rho) \dot{\mathbf{x}}_{r}-k_{0} \tilde{\mathbf{p}}
$$

where $k_{o}>0$, we get:

$$
\dot{V}_{p}=-k_{o} \tilde{\mathbf{p}}^{T} \tilde{\mathbf{p}}+\tilde{\mathbf{p}}^{T} \mathbf{L}^{T} \mathbf{J}(\gamma) \mathbf{H}(\rho) \mathbf{z}_{2}
$$

Unfortunately a cross term in $\tilde{\mathbf{p}}$ and $\mathbf{z}_{2}$ will appear in the expression for $\dot{V}_{3 r}$ if (230) is applied. In order to guarantee that $\dot{V}_{3 r} \leqslant 0$, we must modify the weather optimal controller (204) and add a negative term $-\tilde{\mathbf{p}}^{T} \tilde{\mathbf{p}}$ to the expression:

$$
\dot{V}_{3 r}=-\mathbf{z}_{1 r}^{T}\left(\mathbf{E K}_{p} \mathbf{E}^{T}\right)\left(\mathbf{E} \Lambda \mathbf{E}^{T}\right) \mathbf{z}_{1 r}-\mathbf{z}_{2}^{T}\left(\mathbf{K}_{d}+\mathbf{D}_{x}\right) \mathbf{z}_{2}
$$

to remove the cross term.

\subsubsection{Weather Optimal Position Control (WOPC)}

The cross-terms involving $\tilde{\mathbf{p}}$ and $\mathbf{z}_{2}$ in $\dot{V}_{p}$ can be removed by modifying the nonlinear controller (204) to:

$$
\begin{aligned}
\tau & =\mathbf{T}^{T}\left(\mathbf{M}_{x} \ddot{\mathbf{x}}_{r}+\mathbf{C}_{x} \dot{\mathbf{x}}_{r}+\mathbf{D}_{x} \dot{\mathbf{x}}_{r}-\mathbf{K}_{p} \mathbf{z}_{1}-\mathbf{K}_{d} \mathbf{z}_{2}\right)-\mathbf{q}(\cdot)-\phi \hat{F}_{e} \\
& -\mathbf{T}^{T} \mathbf{E}^{T}(\rho) \mathbf{J}^{T}(\gamma) \mathbf{L} \tilde{\mathbf{p}}
\end{aligned}
$$

The last term in $\tau$ implies that:

$$
\dot{V}_{3 r}=-\mathbf{z}_{1 r}^{T}\left(\mathbf{E} K_{p} \mathbf{E}^{T}\right)\left(\mathbf{E} \Lambda \mathbf{E}^{T}\right) \mathbf{z}_{1 r}-\mathbf{z}_{2}^{T}\left(\mathbf{K}_{d}+\mathbf{D}_{x}\right) \mathbf{z}_{2}-\tilde{\mathbf{p}}^{T} \mathbf{L}^{T} \mathbf{J}(\gamma) \mathbf{H}(\rho) \mathbf{z}_{2}
$$

Consider the LFC:

$$
V_{\text {wopc }}=V_{3 r}+V_{p}
$$

Hence:

$$
\dot{V}_{\text {wopc }}=-\mathbf{z}_{1 r}^{T}\left(\mathbf{E} K_{p} \mathbf{E}^{T}\right)\left(\mathbf{E} \Lambda \mathbf{E}^{T}\right) \mathbf{z}_{1 r}-\mathbf{z}_{2}^{T}\left(\mathbf{K}_{d}+\mathbf{D}_{x}\right) \mathbf{z}_{2}-k_{o} \tilde{\mathbf{p}}^{T} \tilde{\mathbf{p}}
$$

and therefore the equilibrium point $\left(\mathbf{z}_{1 r}, \mathbf{z}_{2}, \tilde{F}_{e}, \tilde{\mathbf{p}}\right)=(\mathbf{0 , 0}, 0, \mathbf{0})$ of the reduced order system is ULES.

The term $\ddot{\mathbf{p}}_{0}$ is needed in the expression for $\mathbf{q}(\cdot)$. This term is computed from (230) as:

$$
\ddot{\mathbf{p}}_{0}=\ddot{\mathbf{p}}_{d}-k_{o}\left(\dot{\mathbf{p}}-\dot{\mathbf{p}}_{d}\right)-\mathbf{L}^{T} \mathbf{J}(\gamma) \mathbf{H}(\rho) \ddot{\mathbf{x}}_{r}-\mathbf{L}^{T} \mathbf{J}(\gamma) \mathbf{H}(\rho) \dot{\mathbf{x}}_{r}-\mathbf{L}^{T} \mathbf{J}(\gamma) \dot{\mathbf{H}}(\rho) \dot{\mathbf{x}}_{r}
$$

\subsection{Passivity Interpretation of the WOPC}

The WOPC is in fact a passivation design. This can be seen from the total system which is governed by the equations:

$$
\begin{aligned}
\dot{\mathbf{z}}_{1} & =-\Lambda \mathbf{z}_{1}+\mathbf{z}_{2} \\
\mathbf{M}_{x} \dot{\mathbf{z}}_{2}+\left(\mathbf{C}_{x}+\mathbf{D}_{x}+\mathbf{K}_{d}\right) \mathbf{z}_{2}+\mathbf{K}_{p} \mathbf{z}_{1} & =-\mathbf{T}^{-T} \phi \tilde{F}_{e}-\mathbf{H}^{T}(\rho) \mathbf{J}^{T}(\gamma) \mathbf{L} \tilde{\mathbf{p}} \\
\tilde{\tilde{F}}_{c} & =\sigma \phi^{T} \mathbf{T}^{-1} \mathbf{z}_{2}
\end{aligned}
$$




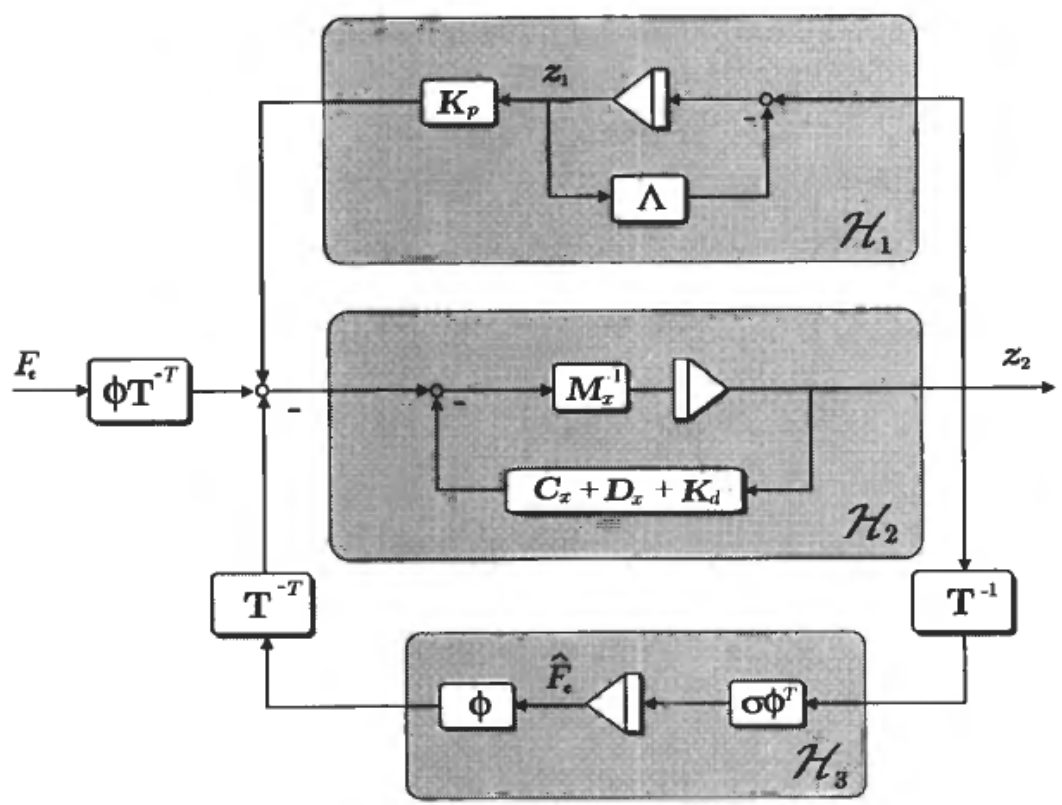

Figure 21. Passive blocks of the positioning controller.

$$
\dot{\tilde{\mathbf{p}}}=-k_{o} \tilde{\mathbf{p}}+\mathbf{L}^{T} \mathbf{J}(\gamma) \mathbf{H}(\rho) \mathbf{z}_{2}
$$

Block diagrams of the passive interconnected systems are shown in Figures 21 and 22.

\subsection{Experimental Results}

The experimental set-up is shown in Figure 23. The experimental results are transformed to full scale according to Table 1 in Section 3.6. In the scaling we used $L_{s}=70 L_{m}$ meters and $m_{s}=4500$ tones. A ducted fan is used to generate a slowlyvarying or constant wind disturbance. A video from Norwegian national TV showing the experiment is available at URL: http://www.itk.ntnu.no

\subsubsection{Experiment 1: Weather Optimal Heading Control (WOHC)}

In the first experiment the ship was allowed to move on the circle arc (circle center controller was turned off). This is referred to as WOHC. The fixed origin and circle arc are shown in Figure 24. Notice that the initial heading is approximately 30 degrees, see Figure 26 , while the position $(x, y) \approx(13,-43)$, see Figure 25 . These values are the ones obtained when the fan was initially directed in 210 degrees (opposite direction of the ship heading).

After 3000 seconds the fan was slowly rotated to 165 degrees corresponding to a weather optimal heading of -15 degrees, see Figure 26. During this process, the ship starts to move on the circle arc (with heading towards the circle center) until it is stabilized to its new heading, that is -15 degrees. The new position on the circle arc is $(x, y) \approx(3,20)$. This clearly demonstrates that the ship heading converges to the optimal value (copies the dynamics of a pendulum in the gravity field). This is done without using any external wind sensor. 


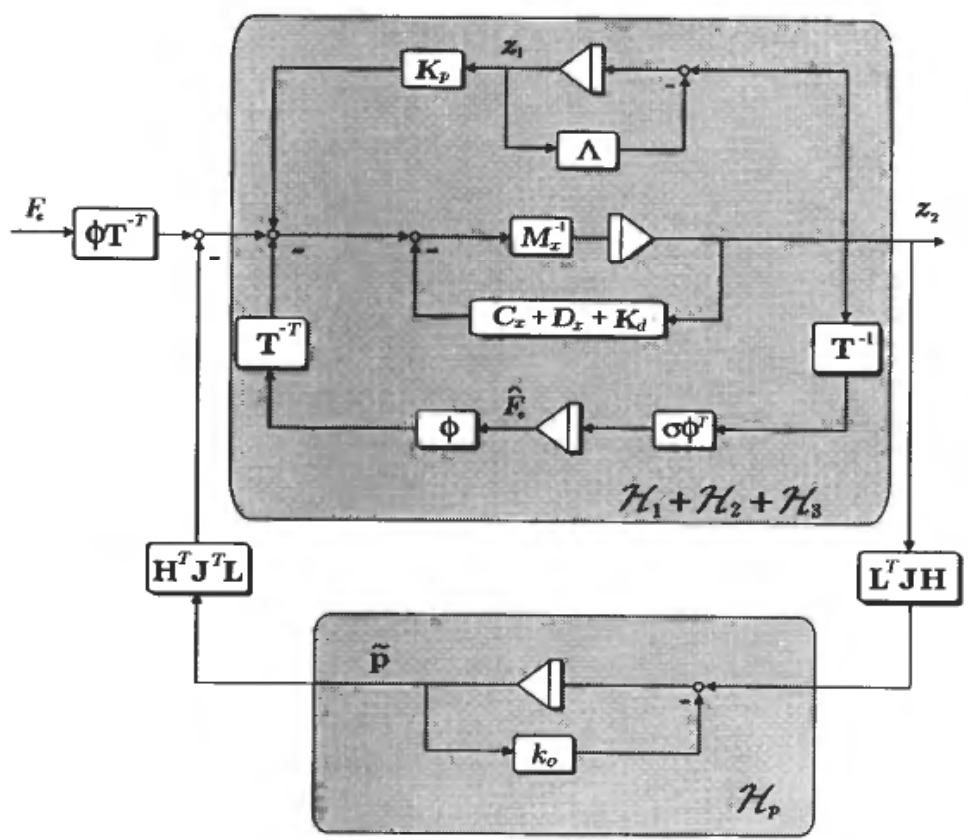

Figure 22. The weather optimal position controller formulated as a passive interconnected system.

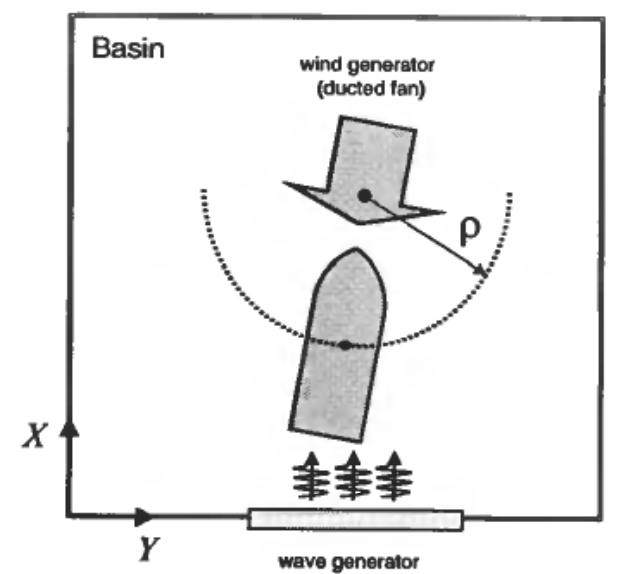

Figure 23. Experimental set-up showing the directions of the wind and wave generators.

In the next experiment, we will show how the circle center can be translated online in order to obtain a constant position $(x, y)$.

\subsubsection{Experiment 2: Weather Optimal Position Control (WOPC)}

In the second experiment the ship should maintain its position (circle center controller is turned on). This is referred to as WOPC. The performance during stationkeeping (dynamic positioning) is shown in Figure 27 while the translation of the circle 


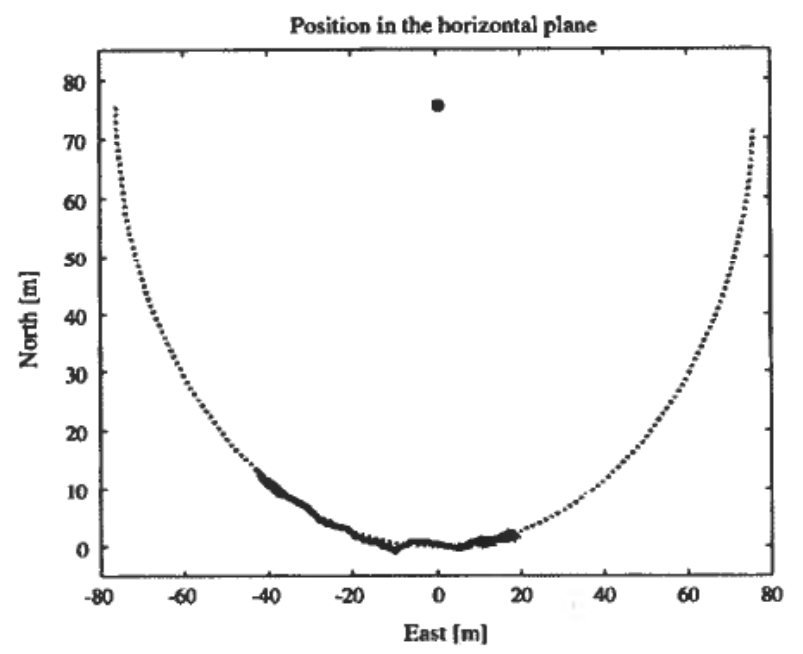

Figure 24. WOHC experiment showing the circular motion of the ship when the circle center controller is turned off (WOHC).

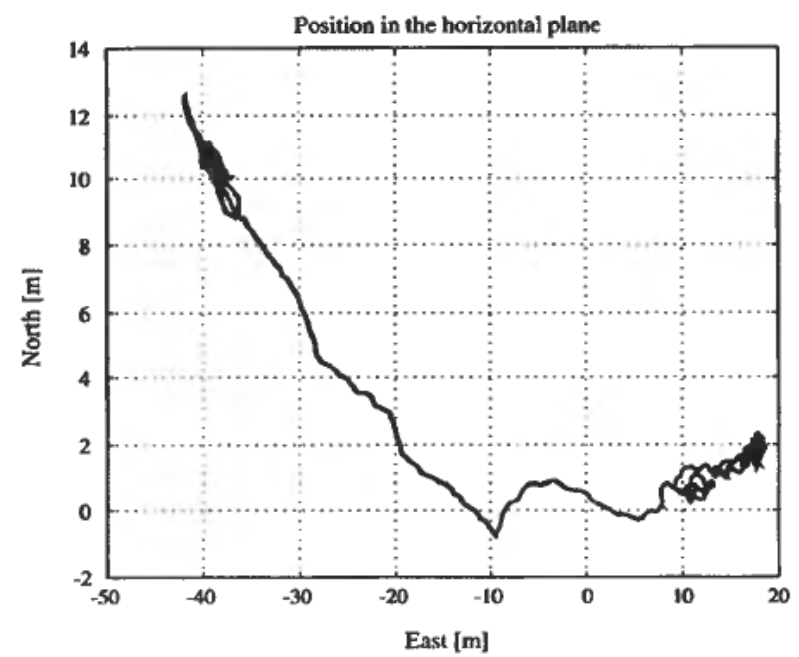

Figure 25. WOHC experiment showing the $(x, y)$ position of the model ship $(\mathrm{m})$ when the fan is rotated.

is shown in Figure 28. The position controller works within an accuracy of \pm 1 meters which is the accuracy of the position reference systems.

Again the weather optimal heading is changed from approximately 23 degrees to 2 degrees but this time without changing the position $(x, y)$ of the ship. The position deviations and the weather optimal heading are shown in Figure 29. These values are obtained by moving the fan from an initial angle of 203 degrees to 182 degrees.

The last plots (Figure 30) shows the deviation for the radius regulator (upper plot) and then how the circle center $\left(x_{0}, y_{0}\right)$ is changed on-line by the circle center control law (lower plots) in order to obtain a fixed position $(x, y)$. The experiment shows that the ship will turn up against an unknown disturbance (wind) at the same time as the ship maintains its position. 

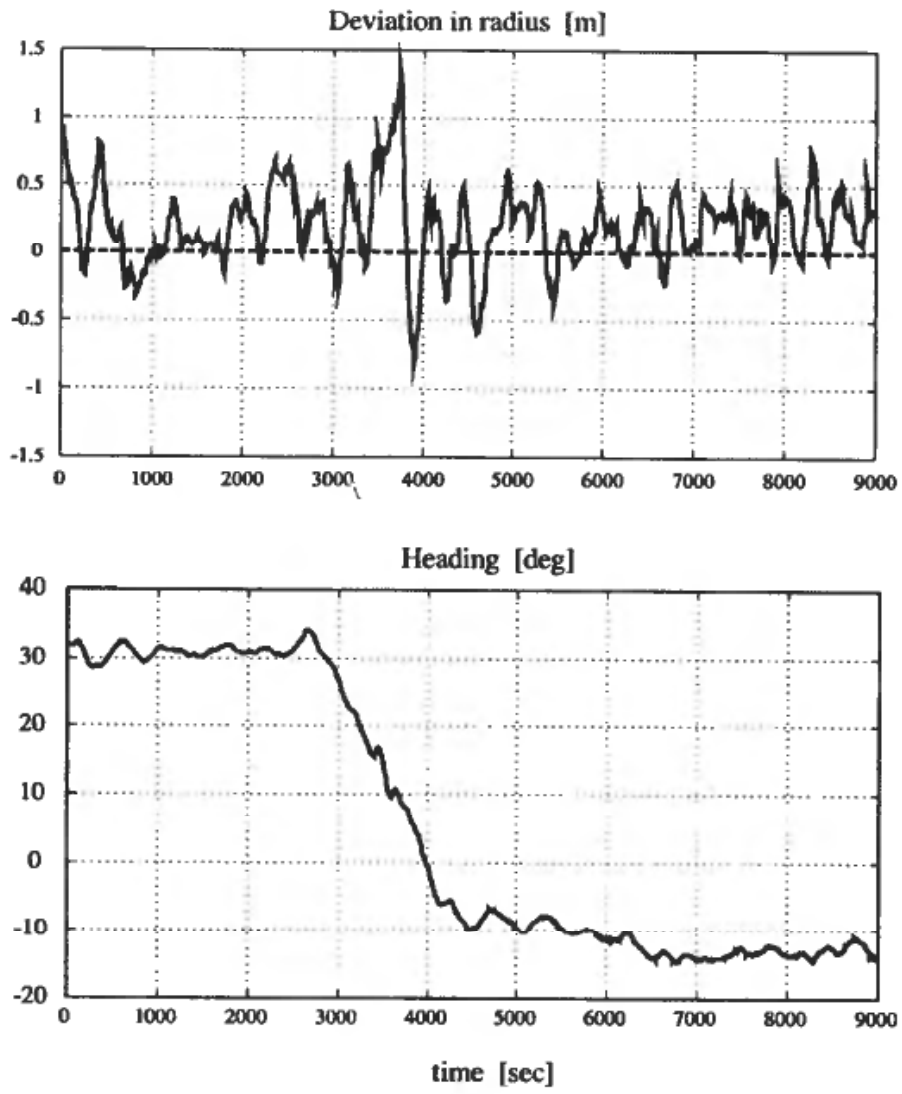

Figure 26. WOHC experiment showing the performance of the radius regulator (upper plot) and weather optimal heading (lower plot) versus time (s).

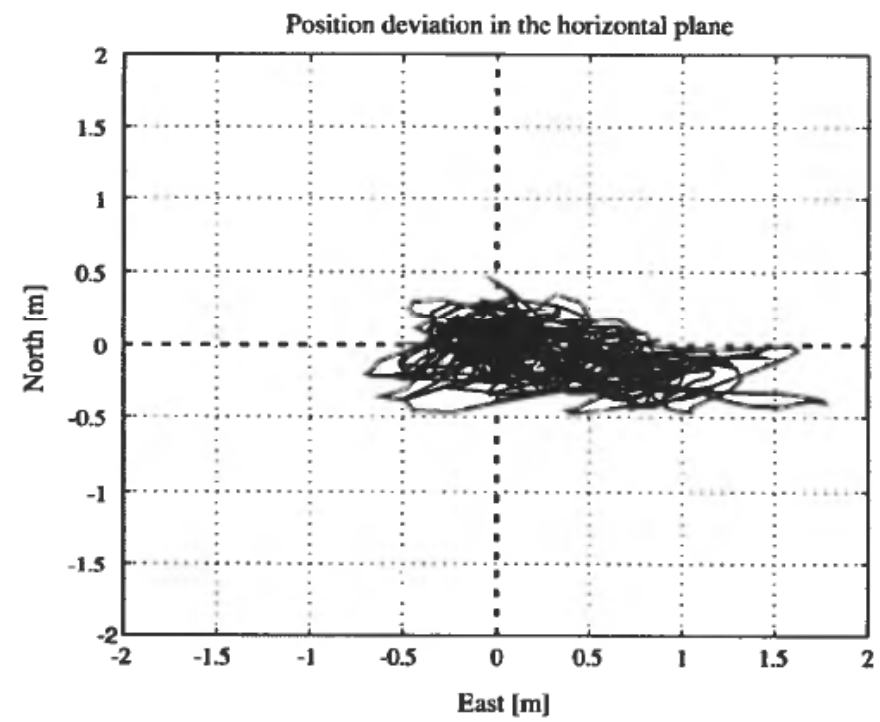

Figure 27. WOPC experiment showing station-keeping to $\left(x_{d}, y_{d}\right)=(0,0)$. 


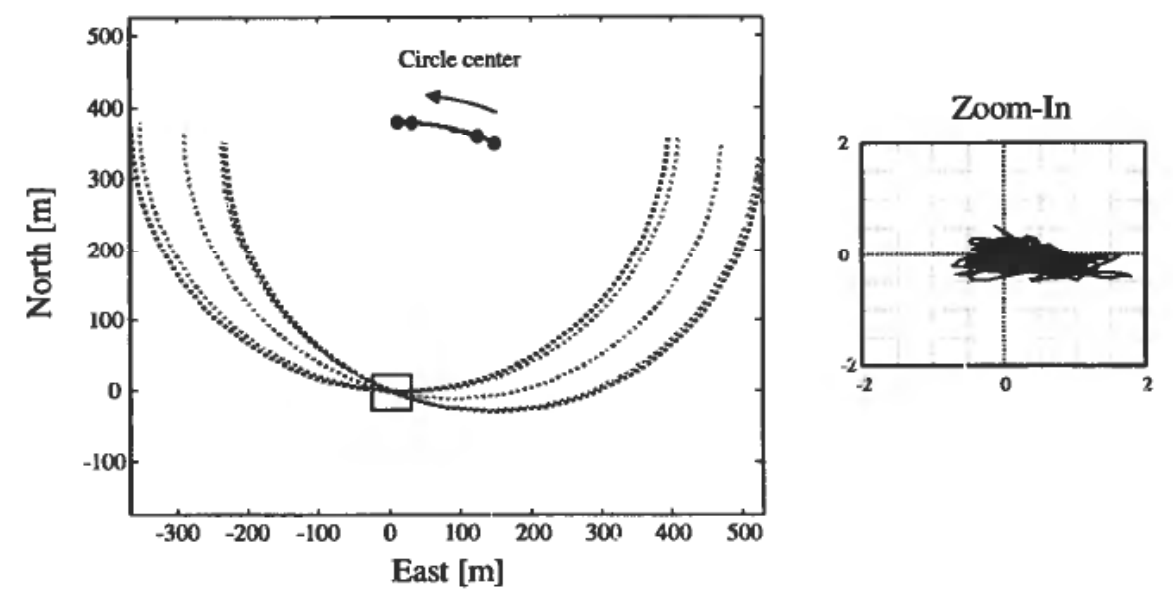

Figure 28. WOPC experiment showing how the circle center is moving during station-keeping.

\section{Conclusions}

In this report the most recent results on passivation designs for ships have been discussed. This includes methods for:

- Nonlinear modeling of ships

- Passive observer design

- Passive dynamic positioning (DP) systems

- Weather optimal passive DP

- Experimental results

\section{Appendix A: Definitions}

The definitions of dissipativity and passivity used in this article are adopted from Sepulchre et al. (1997).

Definition D1: Dissipativity: Assume that associated with the system $H$ with input $u \in \mathscr{R}^{m}$ and output $y \in \mathscr{R}^{m}$ is a function $w: \mathscr{R}^{m} \times \mathscr{R}^{m} \rightarrow \mathscr{R}$, called supply rate, which is locally integrable for every $u \in \mathscr{R}^{m}$, that is, it satisfies $\int_{t_{0}}^{t_{1}}|w(u(t), y(t))| d t<\infty$ for all $t_{0}<t_{1}$. Let $X$ be a connected subset of $\mathscr{R}^{n}$, where $n$ is the number of states and which contains the origin. We say that the system is dissipative in $X$ with the supply rate $w(u, y)$ if there exists a function $S(x), S(0)=0$, such that for all $x \in X$

$$
S(x) \geqslant 0
$$

and

$$
S(x(T))-S(x(0)) \leqslant \int_{0}^{T} w(u, y) d t
$$

for all $u \in \mathscr{R}^{m}$ and $T \geqslant 0$ such that $x(t) \in X$ for all $t \in[0, T]$. The function $S(x)$ is then called a storage function.

Definition D2: Passive: The system $H$ is said to be Passive if it is dissipative with the supply rate $w(u, y)=u^{T} y$. 

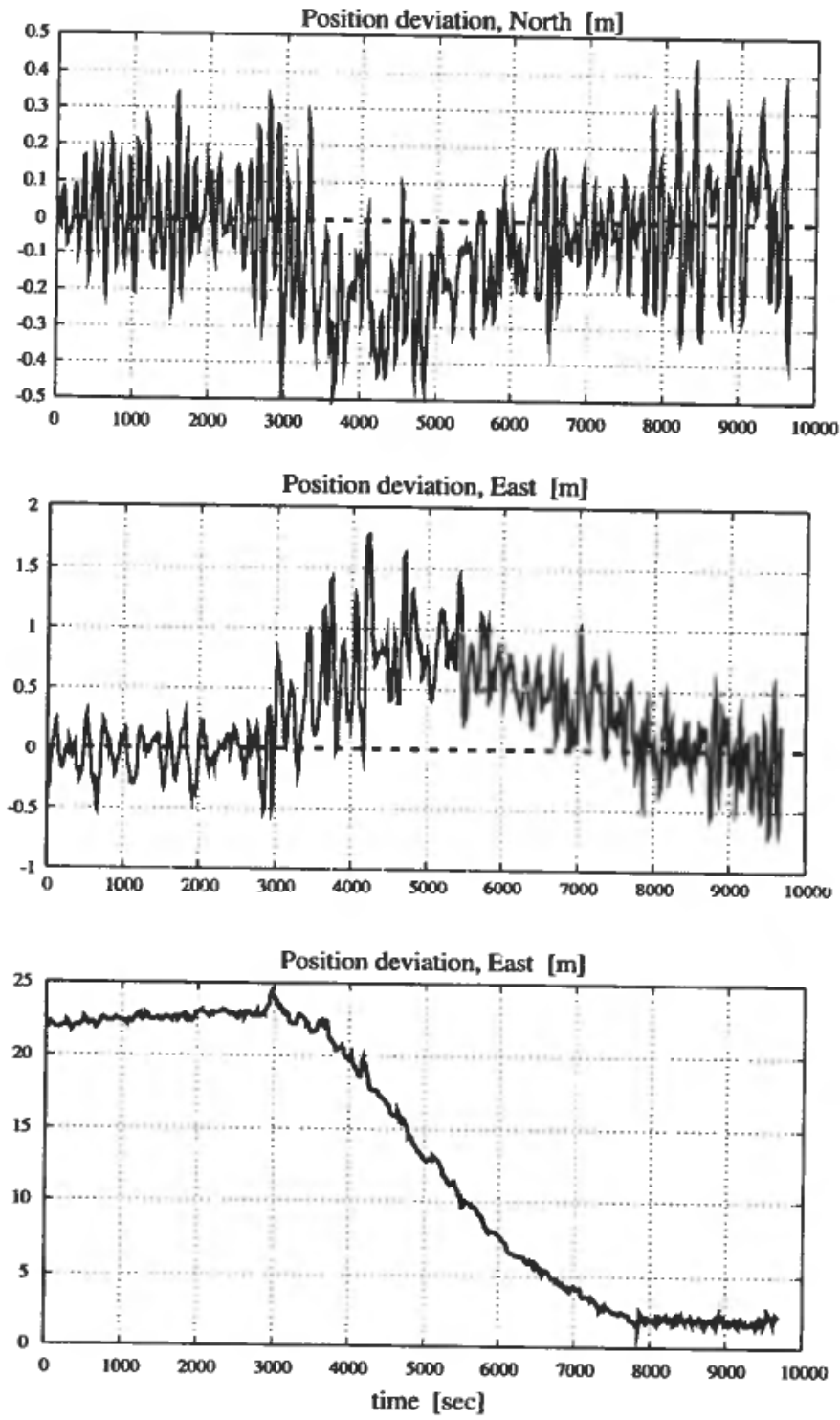

Figure 29. WOPC experiment showing the North and East position accuracies (upper plots) and weather optimal heading (lower plot) versus time (seconds). The position accuracy is within $\pm 1 \mathrm{~m}$ while the heading changes from 23 degrees to 2 degrees as the fan is rotated.

Definition D3: Input-Feedforward Passive: The system $H$ is said to be Input-Feedforward Passive (IFP) or Input Strict Passive if it is dissipative with supply rate $w(u, y)=u^{T} y-\lambda u^{T} u$ for some $\lambda \in \mathscr{R} . \lambda>0$ means that the system has an excess of passivity and $\lambda<0$ means that the system has a shortage of passivity.

Definition D4: Output-Feedback Passive: The system $H$ is said to be Output-Feedback Passive (OFP) or Output Strict Passive if it is dissipative with supply rate $w(u, y)=u^{T} y-\rho y^{T} y$ for some $\rho \in \mathscr{R} . \rho>0$ means that the system has an excess of passivity and $\rho<0$ means that the system has a shortage of passivity. 

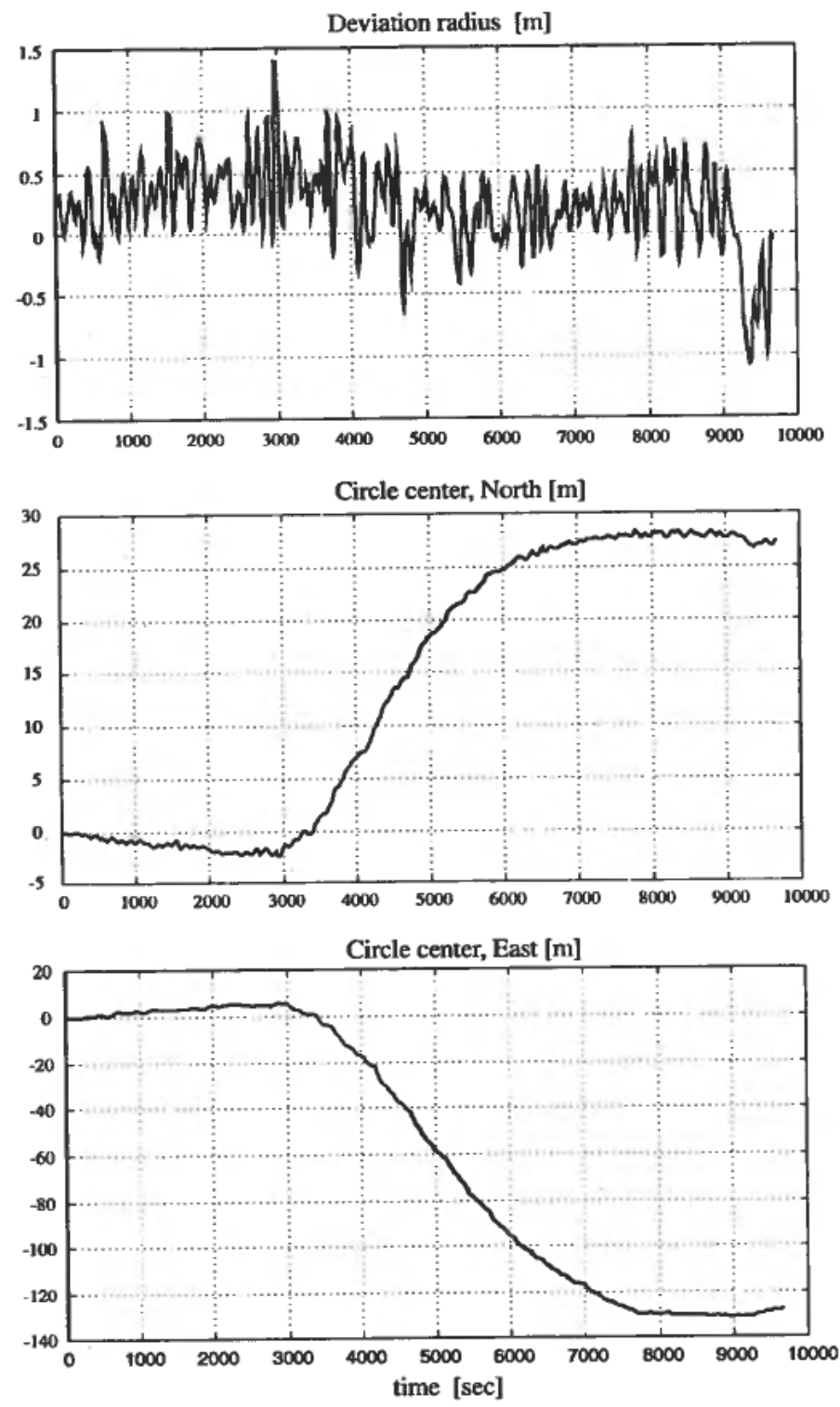

Figure 30. WOPC experiment showing the deviation for the radius regulator (upper plot) and the translation of the circle center $\left(x_{0}, y_{0}\right)$ (lower plots) versus time in seconds. The radius deviation is within $\pm 1 \mathrm{~m}$ during the rotation of the fan.

\section{REFERENCES}

Aarset, M. F., Strand. J. P. and Fossen, T. I. (1998). Nonlinear Vectorial Observer Backstepping With Integral Action and Wave Filtering for Ships. Proceedings of the IFAC Conference on Control Applications in Marine Systems (CAMS'98), Fukuoka, Japan, pp. 83-89.

AвкоWITZ, M. A. (1988). Measurement of Hydrodynamic Characteristics from Ship Maneuvering Trials by System Identification. Transactions on SNAME, 88, pp. 283-318.

Berge, S. P., OHTSU, K. and FosSEN, T. I. (1998). Nonlinear Tracking Control of Underactuated Ships Minimizing the Cross-Track Error, Proceedings of the IFAC Conference on Control Applications in Marine Systems (CAMS'98), Fukuoka, Japan, October 27-30, pp. 141147. 
Berge, S. P. (1999). Nonlinear Way-Point Tracking Control and Docking of Ships. Doctoral Dissertation. Department of Engineering Cybernetics, NTNU, Trondheim, Norway, August 1999.

BerghuIS, H. (1993). Model-based Robot Control: from Theory to Practice, PhD Dissertation, Univ. Twente, Enschede, The Netherlands.

Berghuis, H. and Nimmeiser, H. (1993). A Passivity Approach to Controller-Observer Design for Robots. IEEE Transactions on Robotics and Automation, RA-8(6), pp. 740-754.

Canudas de Wit, C., Fixot, N. and Astrom, K. J. (1992). Trajectory Tracking in Robot Manipulators via Nonlinear Estimated State Feedback. IEEE Transactions on Robotics and Automation, RA-8, pp.138-144.

FALTinsen, O. M. (1993). Sea Loads on Ships and Offshore Structures. John Wiley \& Sons Ltd. Fossen, T. I. (1994). Guidance and Control of Ocean Vehicles. John Wiley \& Sons Ltd.

Fossen, T. I. AND FJellstad, O.-E. (1995) Nonlinear Modelling of Marine Vehicles in 6 Degrees of Freedom. International Journal of Mathematical Modelling of Systems, JMMM-1, pp. 17-28.

Fossen, T. I. and Grøvlen, Å. (1998). Nonlinear Output Feedback Control of Dynamically Positioned Ships Using Vectorial Observer Backstepping. IEEE Transactions on Control Systems Technology, January, TCST-6(1), pp. 121-128.

Fossen, T. I., LoRIA, A. and TeEL, A. (2000). A Theorem for UGAS and ULES of Nonautonomous Systems: Robust Control of Mechanical Systems and Ships. International Journal of Robust and Nonlinear Control, to appear.

Fossen, T. I. and Strand, J. P. (1999). Passive Nonlinear Observer Design for Ships Using Lyapunov Methods: Full-Scale Experiments With a Supply Vessel. Automatica (Regular Paper). AUT-35(1), pp. 3-16.

Fossen, T. I. and STRAND, J. P. (2000). Nonlinear Passive Weather Optimal Positioning Control (WOPC) System for Ships and Rigs: Experimental Results. Automatica, to appear.

Godhavn, J.-M., Fossen, T. I. and BerGe, S. P. (1998). Adaptive Backstepping Designs for Tracking Control of Ships, Special Issue on Marine Systems of the International Journal of Adaptive Control and Signal Processing, JACSP-12, pp. 649-670.

Grøvlen, A. and Fossen, T. I. (1996). Nonlinear Control of Dynamic Positioned Ships Using Only Position Feedback: An Observer Backstepping Approach, Proceedings of the 35th Conference on Decision and Control (CDC'96), Kobe, Japan, 11-13 December 1996.

Hill, D. and Moylan, P. (1977). Stability Results for Nonlinear Feedback Systems. Automatica, AUT-13, pp. 377-382.

KhaliL, H. K. (1996). Nonlinear Systems. Prentice Hall Inc.

Krstic, M., Kanellakopoulos, I. and Кокотоvic, P. V. (1995). Nonlinear and Adaptive Control Design. John Wiley \& Sons Ltd., New York.

LandaU, I. D. and Horowitz, R. (1989). Applications of the Passive Systems Approach to the Stability Analysis of Adaptive Controllers for Robot Manipulators, International Journal of Adaptive Control and Signal Processing, JACSP-3, pp. 23-38.

Lewis, E. V. (Ed.) (1988). Principles of Naval Architecture, The Society of Naval Architects and Marine Engineers, 601 Pavonia Avenue, Jersey City, NJ.

Loria, A., Panteley, E., Nijmeijer, H. and Fossen, T. I. (1998). Robust Adaptive Control of Passive Systems with Unknown Disturbances, Preprints of the IFAC NOLCOS'98, Enschede, The Netherlands, 1-3 July 1998, pp. 866-892.

Loria, A., Fossen, T. 1. and PANTEley, E. (2000). A Cascaded Approach to a Separation Principle for Dynamic Ship Positioning. IEEE Transactions on Control Systems Techno$\log y$, to appear.

Loria, A., Fossen, T. I. and Teel, A. (1999). UGAS and ULES of Non-Autonomous Systems: Applications to Integral Action Control of Ships and Manipulators, Proceedings of the 5th European Control Conference (ECC'99), Karlsruhe, Germany, September.

Nicosia, S. and TomeI, P. (1990). Robot Control by Using only Joint Position Measurements. IEEE Transactions on Automatic Control, AC-35, pp. 1058-1061.

Nicosia, S., Tornambe, A. and VAligi, P. (1990). Experimental Results in State Estimation of Industrial Robots. Proc. Conf. Decision and Control, Honolulu, HI, December, pp. 360365.

Numeijer, H. and Fossen, T. I. (Eds) (1999). New Directions in Nonlinear Observer Design, Springer-Verlag, London. 
OrtegA, R. and SPONG, M. W. (1989) Adaptive Motion Control of Rigid Robots: A Tutorial, Automatica, AUT-25, pp. 877-888.

Ortega, R., Loria, A., Nicklasson, P. and SiRA, H. (1998). Passivity-Based Control of EulerLagrange Systems, Springer-Verlag.

Panteley, E. and Loria, A. (1998). On Global Uniform Asymptotic Stability of Nonlinear Time-Varying Systems in Cascade. Systems \& Control Letters, SCL-33, pp. 131-138.

Paulsen, M. J. (1996). Nonlinear Control of Marine Vehicles using only Position and Attitude Measurements. Doctoral Dissertation, Department of Engineering Cybernetics, NTNU, Trondheim, Norway, April.

Paulsen, M. J., Egeland, O. and Fossen, T. I. (1998). A Passive Feedback Controller With Wave Filter for Marine Vehicles, International Journal of Robust and Nonlinear Control, JRNC-8(15), pp. 1239-1253.

Pinkster, J. A. and Nienhuis, U. (1996). Dynamic Positioning of Large Tankers at Sea. Proceedings of the Offshore Technology Conference (OTC'96), Houston, TX.

Sepulchre, R., Jankovic, M. and Kokotovic, P. V. (1997). Constructive Nonlinear Control. Springer Verlag.

Slotine, J. J.-E., Hedrick, J. K. and Misawa, E. A. (1987). Sliding Observers for Nonlinear Systems. ASME Journal of Dynamic Systems, Measurement, Control, JDSMC-109, pp. $245-252$.

SLotine, J. J.-E. and LI, W. (1987). On the Adaptive Control of Robot Manipulators. International Journal of Robotics Research, JRS-6, pp. 49-59.

Strand, J. P. and Fossen, T. I. (1998). Nonlinear Output Feedback and Locally Optimal Control of Dynamically Positioned Ships: Experimental Results. Proceedings of the IFAC Conference on Control Applications in Marine Systems (CAMS'98), Fukuoka, Japan, pp. $89-95$.

Strand, J. P., Ezal, K., Fossen, T. I. and Kokotovic, P. V. (1998). Nonlinear Control of Ships: A Locally Optimal Design, Preprints of the IFAC NOLCOS'98, Enschede, The Netherlands, 1-3 July 1998, pp. 732-738.

Strand, J. P., Ezal, K., Fossen, T. I. and Kokotovic, P. V. (1999). Nonlinear Output Feedback Control of Free-Floating and Moored Ships: A Locally Optimal Approach. Submitted as a regular paper to Automatica.

Strand, J. P. and Fossen, T. I. (1999). Nonlinear Passive Observer for Ships with Adaptive Wave Filtering, In "New Directions in Nonlinear Observer Design" (H. NIJMEJER and T. I. FoSSEN, Eds), Springer-Verlag London Ltd, pp. 113-134.

Strand, J. P. (1999). Nonlinear Position Control Systems Design for Marine Vessels. Doctoral Dissertation, Department of Engineering Cybernetics, NTNU, Trondheim, Norway, June.

Sørensen, A. J. (1993). Modelling and Control of SES Dynamics in the Vertical Plane, Department of Engineering Cybernetics, Norwegian Institute of Technology, Trondheim.

Sørensen, A. J. and Egeland, O. (1993). Ride Control of Surface Effect Ships using Distributed Control. Proceedings of the IFAC World Congress, 1993, Sydney.

Sørensen, A. J. and Egeland, O. (1995). Design of Ride Control System for Surface Effect Ships using Dissipative Control (Regular Paper), February, Automatica, AUT-31(2), pp. 183-199.

Sørensen, A. J., Sagatun, S. I. and Fossen, T. I. (1996). Design of a Dynamic Positioning System using Model-Based Control. Journal of Control Engineering Practice, CEP-4(3), pp. 359-368.

Willems, J. (1972). Dissipative Dynamical Systems, Part I: General Theory. Arch. Rational Mech. Anal., 45, pp. 321-351. 